\title{
Electromagnetic scattering by discrete random media. I: The dispersion equation and the configuration-averaged exciting field
}

\author{
Adrian Doicu ${ }^{\mathrm{a}, *}$, Michael I. Mishchenko ${ }^{\mathrm{b}}$ \\ ${ }^{a}$ Deutsches Zentrum für Luft- und Raumfahrt (DLR), Institut für Methodik der Fernerkundung (IMF), Oberpfaffenhofen 82234, Germany \\ ${ }^{\mathrm{b}}$ NASA Goddard Institute for Space Studies, 2880 Broadway, New York, NY 10025, USA
}

\section{A R T I C L E I N F O}

\section{Article history:}

Received 16 January 2019

Revised 14 March 2019

Accepted 18 March 2019

Available online 19 March 2019

\begin{abstract}
A B S T R A C T
We consider the scattering of a plane electromagnetic wave obliquely incident on a plane-parallel layer of discrete random medium with non-scattering boundaries. We solve the Lax integral equation for the conditional configuration-averaged exciting field coefficients by assuming a special-form solution, that is, by representing the conditional configuration-averaged exciting field coefficients as a linear combination of the coefficients corresponding to an up-going and a down-going wave. This solution representation is supposed to be valid within the whole domain occupied by the particles, even in the close proximity of the boundaries. By balancing the waves with different propagation directions and wavenumbers we derive two homogeneous systems of equations corresponding to the generalized Lorenz-Lorentz law and two inhomogeneous systems of equations corresponding to the generalized Ewald-Oseen extinction theorem. It is shown that (i) the two homogeneous systems of equations of the generalized Lorenz-Lorentz law reduce to a single homogeneous system of equations corresponding to a semi-infinite discrete random medium at normal incidence; (ii) the dispersion equation is direction and polarization independent; and (iii) the two inhomogeneous systems of equations of the generalized Ewald-Oseen extinction theorem can be reduced to two scalar equations by means of the addition theorem for vector spherical harmonics. It is also shown that the same dispersion equation can be obtained without assuming a special-form solution representation in the proximity of the boundaries.
\end{abstract}

(c) 2019 Elsevier Ltd. All rights reserved.

\section{Introduction}

Multiple scattering of acoustic and electromagnetic waves by discrete random media is a fascinating topic which has been covered in detail in several textbooks [1-8]. The journal literature is also extensive. In this context, the following contributions dealing with the scattering of acoustic waves deserve to be mentioned specifically.

1. Foldy [9] analyzed multiple scattering by a random distribution of isotropic point scatterers by using a stochastic approach. This procedure was later generalized by Lax $[10,11]$ to include point scatterers with quite general scattering properties using a quantum-mechanical formalism. Twersky [12] used the same procedure to treat the scattering and reflection of acoustic waves by a rough surface. To reveal the fundamentals of these approaches, let us consider a Foldy-Lax model for the scattering by $N$ particles

\footnotetext{
* Corresponding author.

E-mail address: adrian.doicu@dlr.de (A. Doicu).
}

randomly distributed throughout a volume $V$. This model relies on the deterministic equations

$u=u_{0}+\sum_{i} \mathcal{A}_{0 i} u_{i}$

$u_{i}=u_{0 i}+\sum_{j \neq i} \mathcal{A}_{i j} u_{j}$

where $u$ is the total field, $u_{0}$ is the impressed incident field, $u_{i}$ is the field exciting particle $i$ centered at $\mathbf{R}_{i}, \mathcal{A}_{0 i}=\mathcal{A}_{0 i}\left(\mathbf{R}_{i}\right)$ is a (linear) operator describing the scattering from particle $i$ to the observation point, $u_{0 i}$ is the incident field at the origin of particle $i$, and the operator $\mathcal{A}_{i j}=\mathcal{A}_{i j}\left(\mathbf{R}_{i}, \mathbf{R}_{j}\right)$ describes the scattering from particle $j$ to particle $i$. Taking the configuration average of Eq. (1) under the assumption that the positions of all the particles are equally probable over the volume $V$, i.e., the single particle probability density function is $p\left(\mathbf{R}_{i}\right)=1 / V$, yields

$\langle u\rangle=u_{0}+n_{0} \int \mathcal{A}_{0 i}\left\langle u_{i}\right\rangle_{i} \mathrm{~d}^{3} \mathbf{R}_{i}$, 
where $n_{0}=N / V$ is the number concentration (particle number density), and $\left\langle u_{i}\right\rangle_{i}$ is the conditional configuration average of $u_{i}$ with the position of particle $i$ held fixed. Taking now the configuration average of Eq. (2) with the position of particle $i$ held fixed, gives (cf. Eq. (64) below)

$\left\langle u_{i}\right\rangle_{i}=u_{0 i}+\sum_{j \neq i} \int \mathcal{A}_{i j}\left\langle u_{j}\right\rangle_{i j} p\left(\mathbf{R}_{j} \mid \mathbf{R}_{i}\right) \mathrm{d}^{3} \mathbf{R}_{j}$,

where $\left\langle u_{i}\right\rangle_{i j}$ is the conditional configuration average of $u_{i}$ with the positions of particles $i$ and $j$ held fixed, and $p\left(\mathbf{R}_{j} \mid \mathbf{R}_{i}\right)$ is the conditional probability of finding particle $j$ at the point $\mathbf{R}_{j}$ if it is known that the particle $i$ is at the point $\mathbf{R}_{i}$. This lack of completeness, i.e., the fact that the conditional average of the field with one particle fixed $\left\langle u_{i}\right\rangle_{i}$ is given in terms of the conditional average of the field with two particles fixed $\left\langle u_{j}\right\rangle_{i j}$, is the basic difficulty encountered in this multiple scattering theory. Essentially, we are led to a hierachy of equations which can be truncated at some level by imposing additional closure assumptions. In these closure assumptions, the conditional average is replaced with the corresponding average with one fewer particle held fixed. At the lowest level, the Foldy closure assumption (otherwise known as the effective field approximation) is $\left\langle u_{i}\right\rangle_{i}=\langle u\rangle$, in which case we are led the so-called Foldy integral equation for the coherent field:

$\langle u\rangle=u_{0}+n_{0} \int \mathcal{A}_{0 i}\langle u\rangle \mathrm{d}^{3} \mathbf{R}_{i}$.

At the next level, the Lax quasi-crystalline approximation is $\left\langle u_{j}\right\rangle_{i j}=\left\langle u_{j}\right\rangle_{j}$, in which case Eq. (4) becomes

$\left\langle u_{i}\right\rangle_{i}=u_{0 i}+n_{0} \int \mathcal{A}_{i j}\left\langle u_{j}\right\rangle_{j} p\left(\mathbf{R}_{j} \mid \mathbf{R}_{i}\right) \mathrm{d}^{3} \mathbf{R}_{j}$.

Note that in general, such assumptions are not straightforward to justify.

2. Waterman and Truell [13] attempted to put Lax's formalism for the configuration-averaged exciting field on a more quantitative footing, and also gave a prescription for obtaining the coherent field once the configuration-averaged exciting field has been found. They assumed spherical wave function expansions for the fields, and applied Eq. (6), which is now an integral equation for the conditional configurationaveraged exciting field coefficients, to the case of normal incidence on a semi-infinite medium containing randomly positioned spheres. The integral equation is solved by employing a "disc-shaped exclusion domain" (with its thickness going to zero), and by assuming a special form representation for the expansion coefficients of the configuration-averaged exciting field. The central result of this computation is an expression for the complex wavenumber describing the propagation in the effective medium. The result obtained for the effective wavenumber is valid over the whole frequency spectrum, thus bridging the gap between the low-frequency limit of point scatterers, where Foldy's results are obtained for isotropic scatterers, and the high-frequency limit, where the results are in agreement with the picture given by the geometrical optics.

3. Fikioris and Waterman [14] revised the approach of Waterman and Truell by considering a sphere rather than a disc exclusion. The solution of the Lax integral equation (6) with the hole-correction approximation to the pair correlation function gives rise to the generalized Lorenz-Lorentz law and the generalized Ewald-Oseen extinction theorem. The designation "generalized" emphasizes the fact that the classical laws are obtained for high frequencies when the particle sizes are comparable to the wavelength. The homo- geneous system of equations corresponding to the generalized Lorenz-Lorentz law yields a dispersion equation for the effective wavenumber, while the inhomogeneous system of equations corresponding to the generalized Ewald-Oseen extinction theorem reduces to a single scalar equation and enables the computation of the configuration-averaged exciting field.

4. The results by Foldy and Lax were extended by Twersky [15] to sparse media by deriving a consistent set of integral equations for the coherent field and the correlation function. The scattering properties of a single particle are described by the scattering amplitude. For dense media, the pair correlation function has been introduced in Ref. [16]. Twersky's theory, which provides a clear physical picture of various processes of the multiple-scattering theory, was reviewed in detail by Ishimaru [1]. Using a series expansion method, which gives a systematic and concise formal representation of the multiple-scattering processes based on the elementary use of Feynman-type diagrams, Ishimaru proved the equivalence of the series expansion method and the Twersky integral equations. Moreover, employing Twersky's arguments, Ishimaru solved the integral equations for a layer of sparsely distributed particles, and established the relation between the multiple scattering theory and the radiative transfer theory.

Multiple scattering of electromagnetic waves by discrete random media has been the focus of subsequent relevant works.

1. Fikioris and Waterman [17] generalized the previously developed scalar formalism [14] to vector problems. Vector equations were used throughout and full account was taken of the hole correction approximation involved in the integral equation by transforming volume to surface integrals. As in the scalar case, a dispersion equation for determining the effective wavenumber was established, and an iterative scheme was used to treat interface corrections. In the low frequency limit, explicit results were obtained, and the boundary conditions at the interface were examined in connection with the results for the coherent reflected and transmitted fields.

2. Varadan et al. [18,19], Varadan and Varadan [20], and Bringi et al. [21,22] used a multiple-scattering formalism analogous to that by Fikioris and Waterman [17]. The difference was that instead of the hole-correction approximation, the Percus-Yevik approximation was employed to model the pair correlation function.

3. Tsang and Kong [5,23] considered the general case of a plane electromagnetic wave obliquely incident on a semiinfinite medium with densely packed spherical particles, and showed that in contrast to the generalized Lorenz-Lorentz law, the generalized Ewald-Oseen extinction theorem depends on polarization and direction of propagation of the incident wave. Besides the dispersion equation, Tsang and Kong derived expressions for the reflected and transmitted coherent fields, and computed the incoherent field by means of the distored Born approximation.

4. Mishchenko [24] and Mishchenko et al. [7,8] have used the far-field Foldy equations obtained under the far-field approximation to analyze the scattering by sparse media composed of arbitrarily shaped particles. This analysis applies to a convex (but otherwise arbitrarily shaped) scattering medium, which assures that a wave exiting the medium cannot reenter it. The scattering properties of a single particle are described by the scattering dyadic, in which case the dispersion relation is a dyadic equation for the dyadic propagation 
constant. This result represents the general vector form of the Foldy approximation for the coherent field.

In a series of papers, we aim to present in a consistent manner the theoretical fundamentals of a scattering problem which has received less attention in the literature, specifically, the scattering of a plane electromagnetic wave obliquely incident on a discrete random medium consisting of spherical particles and confined to a layer with non-scattering boundaries. In this first part of the series, our analysis will be focused on the derivation of the dispersion equation for the effective wavenumber and the calculation of the configuration-averaged exciting field. The subsequent three parts will focus on the computation of the coherent field inside and outside the scattering medium (part II); the derivation of a simplified radiative transfer equation for dense media (part III); and the analysis of the incoherent and coherent scattering in backward directions (part IV). Discrete random media with dense and sparse concentrations of particles will be considered. The solution method is standard: it relies on the superposition T-matrix method and spherical statistics, and in particular, on the (conditional) configuration-averaged equations for the total field and the exciting field coefficients.

The composition of this first part of the series is as follows. We first introduce some matrix notation and discuss several basic results which are the starting point for our further analysis. These include the multiple-scattering equations for a group of particles with fixed positions and the equations governing the configuration averaging process. We then proceed to solve the Lax integral equation for the conditional configuration-averaged exciting field coefficients, or equivalently, to derive the equations of the generalized Lorenz-Lorentz law and the generalized Ewald-Oseen extinction theorem, in the case of a layer with densely packed particles and oblique incidence. The paper ends with an exhaustive discussion on possible further developments of the theory.

\section{Matrix notation}

For a compact notation, we introduce column vector spherical wave functions and vector spherical harmonics. Specifically, the vector spherical wave functions $\mathbf{X}_{1,3}(k \mathbf{r})$ are defined by

$\mathbf{X}_{1,3}(k \mathbf{r})=\left[\mathbf{X}_{m n}^{1,3}(k \mathbf{r})\right]=\left[\begin{array}{l}\mathbf{M}_{m n}^{1,3}(k \mathbf{r}) \\ \mathbf{N}_{m n}^{1,3}(k \mathbf{r})\end{array}\right]$,

where $k$ is the wavenumber, $\mathbf{r}$ is the position vector, and $\left(\mathbf{M}_{m n}^{1}(k \mathbf{r}), \mathbf{N}_{m n}^{1}(k \mathbf{r})\right)$ and $\left(\mathbf{M}_{m n}^{3}(k \mathbf{r}), \mathbf{N}_{m n}^{3}(k \mathbf{r})\right)$ are the systems of regular and radiating vector spherical wave functions, respectively. We assume that the host medium containing particles is perfectly nonabsorbing, which implies a real-valued positive $k$. In the spherical unit-vector basis $(\widehat{\mathbf{r}}, \widehat{\boldsymbol{\theta}}(\widehat{\mathbf{r}}), \widehat{\boldsymbol{\varphi}}(\widehat{\mathbf{r}}))$, we have the representation

$\mathbf{X}_{1,3}(k \mathbf{r})=\mathrm{X}_{r}^{1,3}(k \mathbf{r}) \widehat{\mathbf{r}}+\mathrm{X}_{\theta}^{1,3}(k \mathbf{r}) \widehat{\boldsymbol{\theta}}(\widehat{\mathbf{r}})+\mathrm{X}_{\varphi}^{1,3}(k \mathbf{r}) \widehat{\boldsymbol{\varphi}}(\hat{\mathbf{r}})$,

where for $\eta=r, \theta, \varphi$, the column vector $\mathrm{X}_{\eta}^{1,3}(k \mathbf{r})$ is given by

$\mathrm{X}_{\eta}^{1,3}(k \mathbf{r})=\left[\mathrm{X}_{\eta m n}^{1,3}(k \mathbf{r})\right]=\left[\begin{array}{c}M_{\eta m n}^{1,3}(k \mathbf{r}) \\ N_{\eta m n}^{1,3}(k \mathbf{r})\end{array}\right]$,

with, for example, $M_{\eta m n}^{1,3}(k \mathbf{r})$ being the components of $\mathbf{M}_{m n}^{1,3}(k \mathbf{r})$ along the spherical unit vectors. Similarly, the vector spherical harmonics $\mathbf{m}_{m n}(\widehat{\mathbf{r}})$ and $\mathbf{n}_{m n}(\widehat{\mathbf{r}})$ are concatenated into the vector $\mathbf{x}(\widehat{\mathbf{r}})$ as

$\mathbf{x}(\widehat{\mathbf{r}})=\left[\mathbf{x}_{m n}(\widehat{\mathbf{r}})\right]=\left[\begin{array}{c}(-\mathbf{j})^{n} \mathbf{m}_{m n}(\widehat{\mathbf{r}}) \\ \mathbf{j}(-\mathbf{j})^{n} \mathbf{n}_{m n}(\widehat{\mathbf{r}})\end{array}\right]$,

where $\mathrm{j}=\sqrt{-1}$, and we have the representation

$\mathbf{x}(\widehat{\mathbf{r}})=x_{\theta}(\widehat{\mathbf{r}}) \widehat{\boldsymbol{\theta}}(\widehat{\mathbf{r}})+x_{\varphi}(\widehat{\mathbf{r}}) \widehat{\boldsymbol{\varphi}}(\widehat{\mathbf{r}})$,

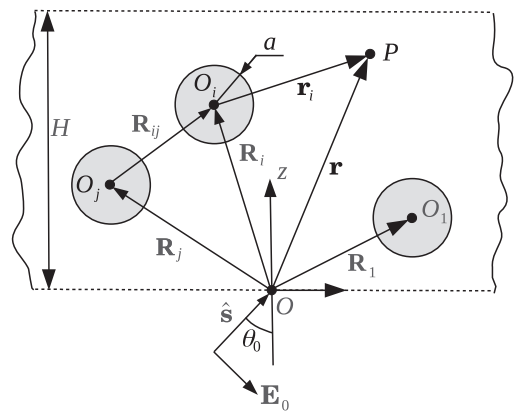

Fig. 1. Scattering by a layer of spherical particles.

with

$\mathrm{x}_{\eta}(\widehat{\mathbf{r}})=\left[\mathrm{x}_{\eta m n}(\widehat{\mathbf{r}})\right]=\left[\begin{array}{c}(-\mathrm{j})^{n} m_{\eta m n}(\widehat{\mathbf{r}}) \\ \mathrm{j}(-\mathrm{j})^{n} n_{\eta m n}(\widehat{\mathbf{r}})\end{array}\right], \quad \eta=\theta, \varphi$.

The vector spherical harmonics and the vector spherical wave functions used in this study are defined in Appendix A. In the farfield region, the radiating vector spherical wave function computes as

$\mathbf{X}_{3}(k \mathbf{r})=-\mathrm{j} \frac{\mathrm{e}^{\mathrm{j} k r}}{k r} \mathbf{x}(\widehat{\mathbf{r}}), \quad k r \rightarrow \infty$,

while the symmetry relations for the vector spherical harmonics imply that

$\mathrm{x}_{\eta m n}^{\star}(-\widehat{\mathbf{r}})=h_{\eta} \times_{\eta-m n}(\widehat{\mathbf{r}})$,

where the asterisk denotes complex conjugation and $h_{\eta}$ is the indicator function

$h_{\eta}=\left\{\begin{array}{l}1, \quad \eta=\theta \\ -1, \eta=\varphi\end{array}\right.$

In terms of the column vector $x_{\eta}(\widehat{\mathbf{r}})$, the elements of the amplitude matrix $\mathbf{S}(\widehat{\mathbf{r}}, \widehat{\mathbf{s}})=\left[S_{\eta \mu}(\widehat{\mathbf{r}}, \widehat{\mathbf{s}})\right]$ are given by

$S_{\eta \mu}(\widehat{\mathbf{r}}, \widehat{\mathbf{s}})=-\frac{4 \pi \mathrm{j}}{k} x_{\eta}^{T}(\widehat{\mathbf{r}}) \mathrm{T}_{\mu}^{\star}(\mathbf{s}), \quad \eta, \mu=\theta, \varphi$,

where $\mathrm{T}$ is the transition matrix of the particle defined with respect to the particle-centered coordinate system. Consequently, the "particle-centered" far-field scattering dyadic $\overline{\mathbf{A}}$, defined by the relation

$\overline{\mathbf{A}}(\widehat{\mathbf{r}}, \widehat{\mathbf{s}})=\sum_{\eta, \mu=\theta, \varphi} S_{\eta \mu}(\widehat{\mathbf{r}}, \widehat{\mathbf{s}}) \widehat{\boldsymbol{\eta}}(\widehat{\mathbf{r}}) \otimes \widehat{\boldsymbol{\mu}}(\widehat{\mathbf{s}})$,

where $\otimes$ is the dyadic product sign, is computed as

$\overline{\mathbf{A}}(\widehat{\mathbf{r}}, \widehat{\mathbf{s}})=-\frac{4 \pi \mathrm{j}}{k} \mathbf{x}^{T}(\widehat{\mathbf{r}}) \mathrm{T} \mathbf{x}^{\star}(\widehat{\mathbf{s}})$.

The addition theorem for radiating vector spherical wave functions plays an important role in the multiple scattering theory. If $\mathbf{r}_{j}$ and $\mathbf{r}_{i}$ are the position vectors of the same field point in coordinate systems $O_{j}$ and $O_{i}$ having identical spatial orientations, respectively, and $\mathbf{R}_{i j}$ is the vector connecting the origins of the two coordinate systems, i.e., $\mathbf{r}_{j}=\mathbf{r}_{i}+\mathbf{R}_{i j}$, then for $r_{i}<R_{i j}$, the addition theorem for radiating vector spherical wave functions reads (Fig. 1)

$\mathbf{X}_{3}\left(k \mathbf{r}_{j}\right)=\mathcal{T}_{31}\left(k \mathbf{R}_{i j}\right) \mathbf{X}_{1}\left(k \mathbf{r}_{i}\right)$.

The translation matrix $\mathcal{T}_{31}\left(k \mathbf{R}_{i j}\right)$ is given by

$$
\begin{aligned}
\mathcal{T}_{31}\left(k \mathbf{R}_{i j}\right) & =\left[\mathcal{T}_{m n, m^{\prime} n^{\prime}}^{31}\left(k \mathbf{R}_{i j}\right)\right] \\
& =\left[\begin{array}{ll}
A_{m n, m^{\prime} n^{\prime}}^{3}\left(k \mathbf{R}_{i j}\right) & B_{m n, m^{\prime} n^{\prime}}^{3}\left(k \mathbf{R}_{i j}\right) \\
B_{m n, m^{\prime} n^{\prime}}^{3}\left(k \mathbf{R}_{i j}\right) & A_{m n, m^{\prime} n^{\prime}}^{3}\left(k \mathbf{R}_{i j}\right)
\end{array}\right],
\end{aligned}
$$


where

$$
\begin{aligned}
& A_{m n, m^{\prime} n^{\prime}}^{3}\left(k \mathbf{R}_{i j}\right)=c_{n n^{\prime}} \sum_{n^{\prime \prime}} \mathrm{j}^{n^{\prime \prime}} a_{1}\left(m, n\left|m^{\prime}, n^{\prime}\right| n^{\prime \prime}\right) u_{m-m^{\prime} n^{\prime \prime}}^{3}\left(k \mathbf{R}_{i j}\right), \\
& B_{m n, m^{\prime} n^{\prime}}^{3}\left(k \mathbf{R}_{i j}\right)=c_{n n^{\prime}} \sum_{n^{\prime \prime}} \mathrm{j}^{n^{\prime \prime}} b_{1}\left(m, n\left|m^{\prime}, n^{\prime}\right| n^{\prime \prime}\right) u_{m-m^{\prime} n^{\prime \prime}}^{3}\left(k \mathbf{R}_{i j}\right),
\end{aligned}
$$

$u_{m n}^{3}\left(k \mathbf{R}_{i j}\right)$ are the radiating spherical wave functions,

$$
\begin{aligned}
a_{1}\left(m, n\left|m^{\prime}, n^{\prime}\right| n^{\prime \prime}\right) & =\int_{0}^{\pi}\left[m m^{\prime} \pi_{n}^{|m|}(\theta) \pi_{n^{\prime}}^{\left|m^{\prime}\right|}(\theta)+\tau_{n}^{|m|}(\theta) \tau_{n^{\prime}}^{\left|m^{\prime}\right|}(\theta)\right] \\
& \times P_{n^{\prime \prime}}^{\left|m-m^{\prime}\right|}(\cos \theta) \sin \theta \mathrm{d} \theta,
\end{aligned}
$$

$$
\begin{aligned}
b_{1}\left(m, n\left|m^{\prime}, n^{\prime}\right| n^{\prime \prime}\right) & =\int_{0}^{\pi}\left[m \pi_{n}^{|m|}(\theta) \tau_{n^{\prime}}^{\left|m^{\prime}\right|}(\theta)+m^{\prime} \tau_{n}^{|m|}(\theta) \pi_{n^{\prime}}^{\left|m^{\prime}\right|}(\theta)\right] \\
& \times P_{n^{\prime \prime}}^{\left|m-m^{\prime}\right|}(\cos \theta) \sin \theta \mathrm{d} \theta,
\end{aligned}
$$

and

$$
c_{n n^{\prime}}=\frac{2 \mathrm{j}^{n^{\prime}-n}}{\sqrt{n n^{\prime}(n+1)\left(n^{\prime}+1\right)}} .
$$

In Eqs. (18)-(21), the summation over $n^{\prime \prime}$ is finite, covering the range $\left|n-n^{\prime}\right|, \ldots, n+n^{\prime}, P_{n}^{|m|}(\cos \theta)$ are the normalized associated Legendre functions, $\tau_{n}^{|m|}(\theta)=\mathrm{d} P_{n}^{|m|}(\cos \theta) / \mathrm{d} \theta$, and $\pi_{n}^{|m|}(\theta)=$ $P_{n}^{|m|}(\cos \theta) / \sin \theta$. Throughout our analysis we will use the symmetry relation

$\mathcal{T}_{m_{1} n_{1}, m n}^{31}\left(k \mathbf{R}_{i j}\right)=\mathcal{T}_{-m n,-m_{1} n_{1}}^{31}\left(-k \mathbf{R}_{i j}\right)$

and the following representation for the translation matrix in the far-field region:

$\mathcal{T}_{31}\left(k \mathbf{R}_{i j}\right)=-4 \pi \mathrm{j} \frac{\mathrm{e}^{\mathrm{j} k R_{i j}}}{k R_{i j}} \mathbf{x}\left(\widehat{\mathbf{R}}_{i j}\right) \cdot \mathbf{x}^{\dagger}\left(\widehat{\mathbf{R}}_{i j}\right), \quad R_{i j} \rightarrow \infty$,

where $\widehat{\mathbf{R}}_{i j}$ is the unit vector in the direction of $\mathbf{R}_{i j}$ and the symbol $\dagger$ stands for complex conjugate transpose.

\section{The superposition T-matrix method}

The notion of a discrete random medium is analyzed in Ref. [25]. We consider a discrete random medium in the form of a group of $N$ identical spherical particles of radius a centered at $\mathbf{R}_{1}, \mathbf{R}_{2}, \ldots, \mathbf{R}_{N}$, and distributed throughout a domain $D$ confined to a laterally infinite plane-parallel layer with the imaginary (nonscattering) boundaries $z=0$ and $z=H$ (see Fig. 1). For simplicity, we assume that the particles are homogeneous and non-magnetic. The permittivities of the non-absorbing, non-magnetic background medium and the particles are $\varepsilon_{1}$ and $\varepsilon_{2}$, respectively, while the wavenumbers are $k_{1}$ and $k_{2}=\mathrm{m} k_{1}$, where $\mathrm{m}$ is the relative refractive index of the particles. We denote by $f=n_{0} V_{0}$ the particle volume concentration, where $n_{0}=N / V$ is the number concentration, $V$ is the volume of the discrete random medium, and $V_{0}=(4 / 3) \pi a^{3}$ is the volume of each particle. If $n_{0}$ is small then the particulate medium is sparse; otherwise the medium is densely populated. Because the medium is infinite in the horizontal directions, we let $V \rightarrow \infty$ and $N \rightarrow \infty$ such that $n_{0}$ remains constant. The particulate medium is illuminated by an impressed incident field [26] in the form of a plane electromagnetic wave with the propagation direction $\widehat{\mathbf{s}}$ and the amplitude $\mathcal{E}_{0}(\widehat{\mathbf{s}})$, that is,

$\left.\mathbf{E}_{0}(\mathbf{r}, t)=\mathbf{E}_{0}(\mathbf{r}) \mathrm{e}^{-\mathrm{j} \omega t}, \mathbf{E}_{0}(\mathbf{r})=\mathcal{E}_{0} \widehat{(\mathbf{s}}\right) \mathrm{e}^{\mathrm{j} k_{1} \widehat{\mathbf{s}} \cdot \mathbf{r}}$,

and

$\mathcal{E}_{0}(\widehat{\mathbf{s}})=\mathcal{E}_{0 \theta} \widehat{\boldsymbol{\theta}}(\widehat{\mathbf{s}})+\mathcal{E}_{0 \varphi} \widehat{\boldsymbol{\varphi}}(\widehat{\mathbf{s}})$. where $t$ is time, $\omega$ is the angular frequency, and $\mathbf{r}$ is the position vector connecting the origin of the laboratory coordinate system and the observation point. The harmonic time dependence will be implicit from now on.

The superposition T-matrix method is a direct corollary of the exact vector Foldy equations $[26,27]$ and can be thought of as reproducing two cooperative effects characterizing the scattering by a group of particles: the interaction between the particles and the superposition of the fields scattered by all particles. The total (electric) field at a position $\mathbf{r}$ sums the contributions of the incident field and of the fields scattered by all the particles:

$\mathbf{E}(\mathbf{r})=\mathbf{E}_{0}(\mathbf{r})+\sum_{i} \mathbf{E}_{\mathrm{scti}}(\mathbf{r})$.

The field exciting particle $i, \mathbf{E}_{\text {exci }}^{(i)}\left(\mathbf{r}_{i}\right)$ is a superposition of the incident field $\mathbf{E}_{0}^{(i)}\left(\mathbf{r}_{i}\right)$ and of the exciting fields $\mathbf{E}_{\text {excij }}^{(i)}$ produced by all particles $j$ except $i$, that is,

$\mathbf{E}_{\text {exci }}^{(i)}\left(\mathbf{r}_{i}\right)=\mathbf{E}_{0}^{(i)}\left(\mathbf{r}_{i}\right)+\sum_{j \neq i} \mathbf{E}_{\text {excij }}^{(i)}\left(\mathbf{r}_{i}\right)$.

Here, the superscript "(i)" indicates that the fields are written in the coordinate system of particle $i$.

In the coordinate system of particle $i$, the field scattered by particle $i$ is expanded in terms of radiating vector spherical wave functions,

$\mathbf{E}_{\mathrm{sct} i}(\mathbf{r})=\mathbf{E}_{\mathrm{sct} i}^{(i)}\left(\mathbf{r}_{i}\right)=\mathbf{X}_{3}^{T}\left(k_{1} \mathbf{r}_{i}\right) \mathrm{s}_{i}, \quad \mathbf{r}=\mathbf{r}_{i}+\mathbf{R}_{i}, \quad r_{i}>a$,

while the field exciting particle $i$ and the incident field are expanded in terms of regular vector spherical wave functions, that is,

$\mathbf{E}_{\mathrm{exci}}^{(i)}\left(\mathbf{r}_{i}\right)=\mathbf{X}_{1}^{T}\left(k_{1} \mathbf{r}_{i}\right) \mathrm{e}_{i}, \quad r_{i}<a$,

and

$\mathbf{E}_{0}^{(i)}\left(\mathbf{r}_{i}\right)=\mathbf{E}_{0}\left(\mathbf{R}_{i}\right) \mathrm{e}^{\mathrm{j} k_{1} \widehat{\mathbf{s}} \cdot \mathbf{r}_{i}}=\mathbf{X}_{1}^{T}\left(k_{1} \mathbf{r}_{i}\right) \mathrm{e}_{0 i}$,

respectively. The vector of the scattered field coefficients $s_{i}$ is related to the vector of the exciting field coefficients $e_{i}$ through the $\mathrm{T}$ matrix, i.e., $\mathrm{s}_{i}=\mathrm{Te}_{i}$, while the vector of the incident field coefficients is given by

$\mathrm{e}_{0 i}=\mathrm{e}^{\mathrm{j} k_{1} \widehat{\mathbf{s}} \cdot \mathbf{R}_{i}} \mathrm{e}_{0}$,

where

$\mathrm{e}_{0}=4 \pi \mathcal{E}_{0}(\widehat{\mathbf{s}}) \cdot \mathbf{x}^{\star}(\widehat{\mathbf{s}})=4 \pi \sum_{\eta=\theta, \varphi} \mathcal{E}_{0 \eta}(\widehat{\mathbf{s}}) \times_{\eta}^{\star}(\widehat{\mathbf{s}})$

is the vector of the incident field coefficients in the global coordinate system.

The contribution of the $j$ th particle to the field exciting particle $i$ is the field scattered by particle $j$ in the coordinate system of particle $i$ :

$\mathbf{E}_{\text {excij }}^{(i)}\left(\mathbf{r}_{i}\right)=\mathbf{E}_{\mathrm{sct} j}^{(i)}\left(\mathbf{r}_{i}\right)=\mathbf{E}_{\mathrm{sct} j}^{(j)}\left(\mathbf{r}_{j}\right), \quad \mathbf{r}_{j}=\mathbf{r}_{i}+\mathbf{R}_{i j}$.

Using the expansion

$\mathbf{E}_{\mathrm{sct} j}^{(j)}\left(\mathbf{r}_{j}\right)=\mathbf{X}_{3}^{T}\left(k_{1} \mathbf{r}_{j}\right) \mathrm{s}_{j}=\mathbf{X}_{3}^{T}\left(k_{1} \mathbf{r}_{j}\right) \mathrm{Te}_{j}$

and the addition theorem for vector spherical wave functions (16), i.e., $\mathbf{X}_{3}\left(k_{1} \mathbf{r}_{j}\right)=\mathcal{T}_{31}\left(k_{1} \mathbf{R}_{i j}\right) \mathbf{X}_{1}\left(k_{1} \mathbf{r}_{i}\right)$ with $\mathbf{R}_{i}=\mathbf{R}_{j}+\mathbf{R}_{i j}$, we obtain

$\mathbf{E}_{\mathrm{excij}}^{(i)}\left(\mathbf{r}_{i}\right)=\mathbf{X}_{1}^{T}\left(k_{1} \mathbf{r}_{i}\right) \mathrm{e}_{i j}$,

where

$\mathrm{e}_{i j}=\mathrm{Q}\left(k_{1} \mathbf{R}_{i j}\right) \mathrm{e}_{j}$

and

$\mathrm{Q}\left(k_{1} \mathbf{R}_{i j}\right)=\mathcal{T}_{31}^{T}\left(k_{1} \mathbf{R}_{i j}\right) \mathrm{T}$. 
From Eq. (28) along with Eqs. (30), (31) and (36), we find (e.g., Ref. [5])

$\mathrm{e}_{i}=\mathrm{e}^{\mathrm{j} k_{1} \hat{\mathbf{s}} \cdot \mathbf{R}_{i}} \mathrm{e}_{0}+\sum_{j \neq i} \mathrm{Q}\left(k_{1} \mathbf{R}_{i j}\right) \mathrm{e}_{j}$.

In fact, Eq. (39) for the exciting field coefficients together with the equation for the total field (cf. Eqs. (27) and (29))

$\mathbf{E}(\mathbf{r})=\mathbf{E}_{0}(\mathbf{r})+\sum_{i} \mathbf{X}_{3}^{T}\left(k_{1} \mathbf{r}_{i}\right) \mathrm{Te}_{i}$

are the multiple scattering equations for densely packed media.

The system of equations (39) can be solved for vertical and horizontal polarizations of the incident field. In this case and in view of Eq. (26), we set

$\mathrm{e}_{0}=\mathcal{E}_{0 \theta} \mathrm{e}_{0 \theta}+\mathcal{E}_{0 \varphi} \mathrm{e}_{0 \varphi}$

where

$\mathrm{e}_{0 \eta}=4 \pi \mathrm{x}_{\eta}^{\star}(\widehat{\mathbf{s}}), \quad \eta=\theta, \varphi$,

and denote by $\mathrm{e}_{i \eta}$ the solution of the system (39) for a $\eta$-polarized incidence, that is,

$\mathrm{e}_{i \eta}=\mathrm{e}^{\mathrm{j} k_{1} \widehat{\mathbf{s}} \cdot \mathbf{R}_{i}} \mathrm{e}_{0 \eta}+\sum_{j \neq i} \mathrm{Q}\left(k_{1} \mathbf{R}_{i j}\right) \mathrm{e}_{j \eta}, \quad \eta=\theta, \varphi$.

Then, it is readily seen that

$\mathrm{e}_{i}=\mathcal{E}_{0 \theta} \mathrm{e}_{i \theta}+\mathcal{E}_{0 \varphi} \mathrm{e}_{i \varphi}$.

In the far-field region, the representation of the translation matrix given by Eq. (24) enable us to express the matrix $Q\left(k_{1} \mathbf{R}_{i j}\right)$ as

$\mathrm{Q}\left(k_{1} \mathbf{R}_{i j}\right)=\frac{1}{k_{1}} g_{0}\left(R_{i j}\right) \mathrm{Q}_{\infty}\left(\widehat{\mathbf{R}}_{i j}\right), \quad R_{i j} \rightarrow \infty$,

where, apart from the normalization factor $4 \pi, g_{0}(r)=\exp \left(\mathrm{j} k_{1} r\right) / r$ is the scalar Green's function for wavenumber $k_{1}$ and

$\mathrm{Q}_{\infty}\left(\widehat{\mathbf{R}}_{i j}\right)=-4 \pi \mathbf{j} \mathbf{x}^{\star}\left(\widehat{\mathbf{R}}_{i j}\right) \cdot \mathbf{x}^{T}\left(\widehat{\mathbf{R}}_{i j}\right) \mathrm{T}$.

Parenthetically, we note that in the framework of the Twersky approximation [15], an order-of-scattering expansion for the exciting field coefficients (actually, the iterated solution of Eq. (39) or the Neumann series) is

$\mathrm{e}_{i}=\mathrm{e}_{0 i}+\sum_{j \neq i} \mathrm{Q}\left(k_{1} \mathbf{R}_{i j}\right) \mathrm{e}_{0 j}+\sum_{j \neq i} \sum_{k \neq i, j} \mathrm{Q}\left(k_{1} \mathbf{R}_{i j}\right) \mathrm{Q}\left(k_{1} \mathbf{R}_{j k}\right) \mathrm{e}_{0 k}+\cdots$,

or in digramatic form

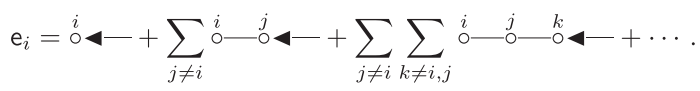

The symbol $4-$ represents the incident field coefficients $e_{0}$, while the symbol - $\circ$ denotes multiplying the field coefficients by the matrix $Q$.

\section{Statistical average}

In this section, we give a brief summary of the probabilistic notions needed. With $\Lambda_{N}=\left\{\mathbf{R}_{1}, \ldots, \mathbf{R}_{N}\right\}$ being a spatial configuration of $N$ particles, let $p\left(\Lambda_{N}\right) \mathrm{d} \Lambda_{N}$ be the probability $p\left(\mathbf{R}_{1}, \ldots, \mathbf{R}_{N}\right) \mathrm{d}^{3} \mathbf{R}_{1} \ldots \mathrm{d}^{3} \mathbf{R}_{N}$ of finding the particles in a configuration wherein the first particle resides inside the volume element $\mathrm{d}^{3} \mathbf{R}_{1}$ centered at the point $\mathbf{R}_{1}$, the second particle resides inside the volume element $d^{3} \mathbf{R}_{2}$ centered at the point $\mathbf{R}_{2}$, and so on up to $\mathbf{R}_{N}$. We assume that the integral of the joint probability density function $p\left(\Lambda_{N}\right)=p\left(\mathbf{R}_{1}, \ldots, \mathbf{R}_{N}\right)$ over all configurations is normalized to unity, and that the $N$ particles are indistinguishable, so that the order of the arguments of $p$ is irrelevant. According to the definition of the probability density function of conditional probabilities, we have

$$
\begin{aligned}
p\left(\Lambda_{N}\right) & =p\left(\mathbf{R}_{i}\right) p\left(\Lambda_{N-1}^{i} \mid \mathbf{R}_{i}\right) \\
& =p\left(\mathbf{R}_{i}\right) p\left(\mathbf{R}_{j} \mid \mathbf{R}_{i}\right) p\left(\Lambda_{N-2}^{i j} \mid \mathbf{R}_{i}, \mathbf{R}_{j}\right) \\
& =p\left(\mathbf{R}_{i}\right) p\left(\mathbf{R}_{j} \mid \mathbf{R}_{i}\right) p\left(\mathbf{R}_{k} \mid \mathbf{R}_{i}, \mathbf{R}_{j}\right) p\left(\Lambda_{N-3}^{i j k} \mid \mathbf{R}_{i}, \mathbf{R}_{j}, \mathbf{R}_{k}\right),
\end{aligned}
$$

where $p\left(\mathbf{R}_{i}\right)$ is the probability of finding particle $i$ at the point $\mathbf{R}_{i} ; p\left(\Lambda_{N-1}^{i} \mid \mathbf{R}_{i}\right)$ with $\Lambda_{N-1}^{i}=\Lambda_{N} \backslash\left\{\mathbf{R}_{i}\right\} \quad$ is the conditional probability of finding the remaining particles at the corresponding points; $p\left(\mathbf{R}_{j} \mid \mathbf{R}_{i}\right)$ is the conditional probability of finding particle $j$ at the point $\mathbf{R}_{j}$ if it is known that the particle $i$ is at the point $\mathbf{R}_{i}$; $p\left(\Lambda_{N-2}^{i j} \mid \mathbf{R}_{i}, \mathbf{R}_{j}\right)$ with $\quad \Lambda_{N-2}^{i j}=\Lambda_{N} \backslash\left\{\mathbf{R}_{i}, \mathbf{R}_{j}\right\} \quad$ is the conditional probability of finding the remaining particles at the corresponding points; and so on. $p\left(\Lambda_{N}\right)$ can also be expressed in terms of the joint probability density functions, i.e.,

$$
\begin{aligned}
p\left(\mathbf{R}_{i}, \mathbf{R}_{j}\right) & =p\left(\mathbf{R}_{i}\right) p\left(\mathbf{R}_{j} \mid \mathbf{R}_{i}\right), \\
p\left(\mathbf{R}_{i}, \mathbf{R}_{j}, \mathbf{R}_{k}\right) & =p\left(\mathbf{R}_{i}, \mathbf{R}_{j}\right) p\left(\mathbf{R}_{k} \mid \mathbf{R}_{i}, \mathbf{R}_{j}\right) \\
& =p\left(\mathbf{R}_{i}\right) p\left(\mathbf{R}_{j} \mid \mathbf{R}_{i}\right) p\left(\mathbf{R}_{k} \mid \mathbf{R}_{i}, \mathbf{R}_{j}\right),
\end{aligned}
$$

where, for example, $p\left(\mathbf{R}_{i}, \mathbf{R}_{j}\right) \mathrm{d}^{3} \mathbf{R}_{i} \mathrm{~d}^{3} \mathbf{R}_{j}$ is the joint probability of finding particle $i$ in $\mathrm{d}^{3} \mathbf{R}_{i}$ and particle $j$ in $\mathrm{d}^{3} \mathbf{R}_{j}$. In particular, if the positions of the particles are independent (i.e., uncorrelated), we have

$$
p\left(\Lambda_{N}\right)=p\left(\mathbf{R}_{i}\right) p\left(\Lambda_{N-1}^{i}\right)=p\left(\mathbf{R}_{i}\right) p\left(\mathbf{R}_{j}\right) p\left(\Lambda_{N-2}^{i j}\right)=\ldots=\prod_{i} p\left(\mathbf{R}_{i}\right)
$$

If the particles are uniformly distributed, the positions of all the particles are equally probable within the volume $V$, and the single particle probability density function is

$p\left(\mathbf{R}_{i}\right)=\frac{1}{V}, \quad i=1, \ldots, N$.

It is a common practice to express the conditional probability $p\left(\mathbf{R}_{j} \mid \mathbf{R}_{i}\right)$ as

$p\left(\mathbf{R}_{j} \mid \mathbf{R}_{i}\right)=\frac{1}{V} g\left(\mathbf{R}_{i}, \mathbf{R}_{j}\right)$,

where $g\left(\mathbf{R}_{i}, \mathbf{R}_{j}\right)$ is the two-point correlation function. For radially symmetric problems, we have $\left(\mathbf{R}_{j i}=\mathbf{R}_{j}-\mathbf{R}_{i}\right)$

$g\left(\mathbf{R}_{i}, \mathbf{R}_{j}\right)=g\left(\mathbf{R}_{j i}\right)=g\left(R_{j i}\right)$,

and $g$ is called the pair correlation function. Because the particles are mutually impenetrable, the pair correlation function for spherical particles of radius $a$ must satisfy the criterion $g\left(R_{j i}\right)=0$ for $R_{j i}<2 a$. Another criterion is that unless the volume concentration $f=n_{0} V_{0}$ is equal to the maximum volume concentration $f_{\max }$, the two particles must be uncorrelated when the separation distance approaches infinity, i.e., $\lim _{R_{j i} \rightarrow \infty} g\left(R_{j i}\right)=1$ for $f<f_{\max }$. In the special case of independent particle positions, the conditional probability $p\left(\mathbf{R}_{j} \mid \mathbf{R}_{i}\right)$ is $p\left(\mathbf{R}_{j} \mid \mathbf{R}_{i}\right)=p\left(\mathbf{R}_{j}\right)=1 / V$, so that the pair correlation function becomes $g\left(R_{j i}\right)=1$. Another approximation to the pair correlation function is the hole-correction approximation, given by

$g\left(R_{j i}\right)= \begin{cases}0, & R_{j i}<2 a \\ 1, & R_{j i} \geq 2 a\end{cases}$

The hole-correction approximation takes into account the fact that the particles cannot penetrate each other and further assumes uniform distribution outside the hole. Neither the independent position approximation nor the hole-correction approximation is correct when the volume concentration of the particles is appreciable. 
A brief insight into the theory of pair correlation functions reveals that the influence of particle $i$ on particle $j$ can be described by the total influence, or the total distribution function $h\left(\mathbf{R}_{j i}\right)=$ $g\left(\mathbf{R}_{j i}\right)-1$. When $g\left(R_{j i}\right)=1$, the particle positions are independent of each other, and obviously, $h\left(R_{j i}\right)=0$. The total influence is decomposed into a sum of a direct and an indirect correlation function. The direct correlation function $C\left(\mathbf{R}_{j i}\right)$ is such that it satisfies the Ornstein-Zernike integral equation [28]

$h\left(\mathbf{R}_{j i}\right)=C\left(\mathbf{R}_{j i}\right)+n_{0} \int C\left(\mathbf{R}_{j k}\right) h\left(\mathbf{R}_{k i}\right) \mathrm{d}^{3} \mathbf{R}_{k}$.

The physical interpretation of the above equation is that the indirect influence of particle $i$ on particle $j$ (the integral term in Eq. (55)) is a result of particle $i$ acting directly on a particle at $\mathbf{R}_{k}$ which in turn exerts the total influence on particle $j$. The total distribution function can be expressed in terms of its Fourier transform $h_{p}(\mathbf{p})$ as

$h(\mathbf{r})=\frac{1}{(2 \pi)^{3}} \int \mathrm{e}^{\mathrm{j} \mathbf{p} \cdot \mathbf{r}} h_{p}(\mathbf{p}) \mathrm{d}^{3} \mathbf{p}$,

so that, assuming spherical symmetry for $h_{p}(\mathbf{p})$, i.e., $h_{p}(\mathbf{p})=h_{p}(p)$, we get

$h(r)=\frac{1}{2 \pi^{2}} \int_{0}^{\infty} \frac{\sin (p r)}{p r} h_{p}(p) p^{2} \mathrm{~d} p$.

The Ornstein-Zernike equation with the Percus-Yevick approximation [29] has a closed-form solution for the case of hard-sphere potential. In the Fourier space, the solution to the Ornstein-Zernike equation (55) is [5]

$h_{p}(p)=\frac{C_{p}(p)}{1-n_{0} C_{p}(p)}$,

where the Fourier transform of the direct correlation function $C_{p}(p)$ is

$$
\begin{aligned}
C_{p}(p) & =\frac{24 f}{n_{0}}\left[\frac{\alpha+\beta+\delta}{u^{2}} \cos u-\frac{\alpha+2 \beta+4 \delta}{u^{3}} \sin u\right. \\
& \left.-\frac{2(\beta+6 \delta)}{u^{4}} \cos u+\frac{2 \beta}{u^{4}}+\frac{24 \delta}{u^{5}} \sin u+\frac{24 \delta}{u^{6}}(\cos u-1)\right],
\end{aligned}
$$

with $u=2 a p$ and

$\alpha=\frac{(1+2 f)^{2}}{(1-f)^{4}}, \quad \beta=-6 f \frac{(1+f / 2)^{2}}{(1-f)^{4}}, \quad \delta=\frac{f \alpha}{2}$.

Thus, the Fourier transform $h_{p}(p)$ can be calculated readily, while the total distribution function is obtained from Eq. (56).

The dense medium theory is developed for media with particles of finite sizes. Hence, the particle positions are correlated, and the Percus-Yevick approximation to the pair correlation function is often employed. For a sparse concentration of particles of finite sizes, it is reasonable to use the hole-correction approximation. In other words, it is reasonable to suppose that the particles do not penetrate each other, but are otherwise uncorrelated. However, in order to simplify the calculations, the particles are assumed to be point scatterers, in which case, the requirement of non-penetration makes no sense. Thus, for sparse media, the assumption of independent particle positions, implying $g\left(R_{j i}\right)=1$, is adopted in the majority of the studies.

The conditional configuration averages of a function $f\left(\mathbf{r}, \Lambda_{N}\right)$ while holding the positions of one, two, and three particles fixed are defined by

$$
\begin{aligned}
\left\langle f\left(\mathbf{r}, \Lambda_{N}\right)\right\rangle_{i} & =\int f\left(\mathbf{r}, \Lambda_{N}\right) p\left(\Lambda_{N-1}^{i} \mid \mathbf{R}_{i}\right) \mathrm{d} \Lambda_{N-1}^{i}, \\
\left\langle f\left(\mathbf{r}, \Lambda_{N}\right)\right\rangle_{i j} & =\int f\left(\mathbf{r}, \Lambda_{N}\right) p\left(\Lambda_{N-2}^{i j} \mid \mathbf{R}_{i}, \mathbf{R}_{j}\right) \mathrm{d} \Lambda_{N-2}^{i j},
\end{aligned}
$$

$\left\langle f\left(\mathbf{r}, \Lambda_{N}\right)\right\rangle_{i j k}=\int f\left(\mathbf{r}, \Lambda_{N}\right) p\left(\Lambda_{N-3}^{i j k} \mid \mathbf{R}_{i}, \mathbf{R}_{j}, \mathbf{R}_{k}\right) \mathrm{d} \Lambda_{N-3}^{i j k}$.

The configuration average of $f\left(\mathbf{r}, \Lambda_{N}\right)$ is then given by

$$
\begin{aligned}
\left\langle f\left(\mathbf{r}, \Lambda_{N}\right)\right\rangle & =\int f\left(\mathbf{r}, \Lambda_{N}\right) p\left(\Lambda_{N}\right) \mathrm{d} \Lambda_{N} \\
& =\int f\left(\mathbf{r}, \Lambda_{N}\right) p\left(\mathbf{R}_{i}\right) p\left(\Lambda_{N-1}^{i} \mid \mathbf{R}_{i}\right) \mathrm{d} \Lambda_{N-1}^{i} \mathrm{~d}^{3} \mathbf{R}_{i} \\
& =\int p\left(\mathbf{R}_{i}\right)\left\langle f\left(\mathbf{r}, \Lambda_{N}\right)\right\rangle_{i} \mathrm{~d}^{3} \mathbf{R}_{i},
\end{aligned}
$$

with (cf. Eq. (51)) $p\left(\mathbf{R}_{i}\right)=1 / V$. Noting that

$$
\begin{aligned}
\left\langle f\left(\mathbf{r}, \Lambda_{N}\right)\right\rangle_{i} & =\int f\left(\mathbf{r}, \Lambda_{N}\right) p\left(\Lambda_{N-1}^{i} \mid \mathbf{R}_{i}\right) \mathrm{d} \Lambda_{N-1}^{i} \\
& =\int f\left(\mathbf{r}, \Lambda_{N}\right) p\left(\mathbf{R}_{j} \mid \mathbf{R}_{i}\right) p\left(\Lambda_{N-2}^{i j} \mid \mathbf{R}_{i}, \mathbf{R}_{j}\right) \mathrm{d}^{3} \mathbf{R}_{j} \mathrm{~d} \Lambda_{N-2}^{i j} \\
& =\int f\left(\mathbf{r}, \Lambda_{N}\right) p\left(\mathbf{R}_{j}, \mathbf{R}_{k} \mid \mathbf{R}_{i}\right) p\left(\Lambda_{N-3}^{i j k} \mid \mathbf{R}_{i}, \mathbf{R}_{j}, \mathbf{R}_{k}\right) \\
& \times \mathrm{d}^{3} \mathbf{R}_{k} \mathrm{~d}^{3} \mathbf{R}_{j} \mathrm{~d} \Lambda_{N-3}^{i j k},
\end{aligned}
$$

with

$p\left(\mathbf{R}_{j}, \mathbf{R}_{k} \mid \mathbf{R}_{i}\right)=p\left(\mathbf{R}_{j} \mid \mathbf{R}_{i}\right) p\left(\mathbf{R}_{k} \mid \mathbf{R}_{i}, \mathbf{R}_{j}\right)$,

we obtain

$$
\begin{aligned}
\left\langle f\left(\mathbf{r}, \Lambda_{N}\right)\right\rangle_{i} & =\int\left\langle f\left(\mathbf{r}, \Lambda_{N}\right)\right\rangle_{i j} p\left(\mathbf{R}_{j} \mid \mathbf{R}_{i}\right) \mathrm{d}^{3} \mathbf{R}_{j} \\
& =\int\left\langle f\left(\mathbf{r}, \Lambda_{N}\right)\right\rangle_{i j k} p\left(\mathbf{R}_{j}, \mathbf{R}_{k} \mid \mathbf{R}_{i}\right) \mathrm{d}^{3} \mathbf{R}_{k} \mathrm{~d}^{3} \mathbf{R}_{j} .
\end{aligned}
$$

For statistically independent particles, Eq. (64) becomes

$$
\begin{aligned}
\left\langle f\left(\mathbf{r}, \Lambda_{N}\right)\right\rangle_{i} & =\int\left\langle f\left(\mathbf{r}, \Lambda_{N}\right)\right\rangle_{i j} p\left(\mathbf{R}_{j}\right) \mathrm{d}^{3} \mathbf{R}_{j} \\
& =\int\left\langle f\left(\mathbf{r}, \Lambda_{N}\right)\right\rangle_{i j k} p\left(\mathbf{R}_{j}\right) p\left(\mathbf{R}_{k}\right) \mathrm{d}^{3} \mathbf{R}_{k} \mathrm{~d}^{3} \mathbf{R}_{j},
\end{aligned}
$$

with $p\left(\mathbf{R}_{j}\right)=p\left(\mathbf{R}_{k}\right)=1 / V$.

\section{The generalized Lorenz-Lorentz law and Ewald-Oseen extinction theorem}

In this section we use the quasi-crystalline approximation to derive an integral equation for the conditional configurationaveraged exciting field coefficients in the case of a layer with densely packed particles and oblique incidence. By assuming a special-form solution, the equations of the generalized LorenzLorentz law and the generalized Ewald-Oseen extinction theorem are obtained. These are solved by employing the technique used by Tsang and Kong for a semi-infinite discrete random medium $[5,23]$.

\subsection{Integral equation for the conditional configuration-averaged exciting field coefficients}

Taking the conditional configuration average of Eq. (39) with the position of particle $i$ held fixed, and using Eq. (64) in conjunction with Eq. (52), yields

$$
\begin{aligned}
\left\langle\mathrm{e}_{i}\right\rangle_{i}\left(\mathbf{R}_{i}\right) & =\mathrm{e}^{\mathrm{j} \mathbf{k}_{1 \mathrm{~s}} \cdot \mathbf{R}_{i}} \mathrm{e}_{0}+n_{0} \int_{D-D_{2 a}\left(\mathbf{R}_{i}\right)} \mathrm{Q}\left(k_{1} \mathbf{R}_{i j}\right) \\
& \times\left\langle\mathrm{e}_{j}\right\rangle_{i j}\left(\mathbf{R}_{i}, \mathbf{R}_{j}\right) g\left(R_{i j}\right) \mathrm{d}^{3} \mathbf{R}_{j},
\end{aligned}
$$

where $D_{2 a}\left(\mathbf{R}_{i}\right)$ is an exclusion domain, $\mathbf{k}_{1 \mathrm{~s}}$ is the wave vector of the incident field,

$\mathbf{k}_{1 \mathrm{~s}}=\mathbf{k}_{1 \mathrm{~s}}\left(\theta_{0}, \varphi_{0}\right)=k_{1} \widehat{\mathbf{s}}=\mathbf{k}_{1 \mathrm{~s} \perp}+k_{1 \mathrm{z}}\left(\mathbf{k}_{1 \mathrm{~s} \perp}\right) \widehat{\mathbf{z}}$,

$\left(\theta_{0}, \varphi_{0}\right)$ are the spherical angles of the incident direction $\widehat{\mathbf{s}}$, and $k_{1 \mathrm{z}}\left(\mathbf{k}_{1 \mathrm{~s} \perp}\right)=\sqrt{k_{1}^{2}-k_{1 \mathrm{~s} \perp}^{2}}$ with $k_{1 \mathrm{~s} \perp}=\left|\mathbf{k}_{1 \mathrm{~s} \perp}\right|$. In Eq. (66) we used the 


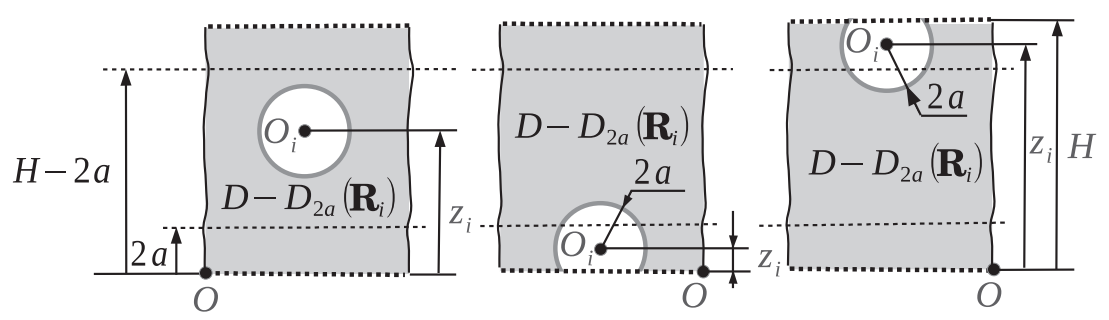

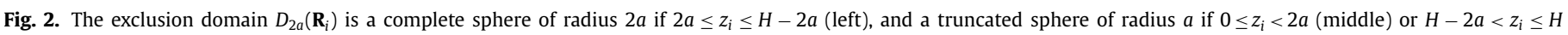
(right).

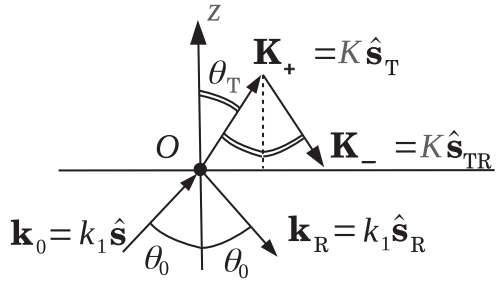

Fig. 3. Wave vectors $\mathbf{k}_{0}, \mathbf{k}_{\mathrm{R}}, \mathbf{K}_{+}$and $\mathbf{K}_{-}$.

fact that $g\left(R_{i j}\right)=0$ for $0 \leq R_{i j}<2 a$, so that

$\int_{D} g\left(R_{i j}\right) \mathrm{d}^{3} \mathbf{R}_{j}=\int_{D-D_{2 a}\left(\mathbf{R}_{i}\right)} g\left(R_{i j}\right) \mathrm{d}^{3} \mathbf{R}_{j}$.

For $2 a \leq z_{i} \leq H-2 a$, where $\mathbf{R}_{i}=\mathbf{R}_{i \perp}+z_{i} \widehat{\mathbf{z}}$ and $\mathbf{R}_{i \perp}=x_{i} \widehat{\mathbf{x}}+y_{i} \widehat{\mathbf{y}}$, the exclusion domain $D_{2 a}\left(\mathbf{R}_{i}\right)$ is a complete sphere of radius $2 a$ centered at $\mathbf{R}_{i}$, while for $0 \leq z_{i}<2 a$ or $H-2 a<z_{i} \leq H$, a truncated sphere of radius $2 a$ is excluded; its size depends on $z_{i}$ (Fig. 2). This observation points to the necessity of a special treatment of these domains for finite scatterers. Invoking the quasi-crystalline approximation,

$\left\langle\mathrm{e}_{j}\right\rangle_{i j}\left(\mathbf{R}_{i}, \mathbf{R}_{j}\right)=\left\langle\mathrm{e}_{j}\right\rangle_{j}\left(\mathbf{R}_{j}\right)$,

yields

$$
\begin{aligned}
\left\langle\mathrm{e}_{i}\right\rangle_{i}\left(\mathbf{R}_{i}\right) & =\mathrm{e}^{\mathrm{j} \mathbf{k}_{1 \mathrm{~s}} \cdot \mathbf{R}_{i}} \mathrm{e}_{0}+n_{0} \int_{D-D_{2 a}\left(\mathbf{R}_{i}\right)} \mathrm{Q}\left(k_{1} \mathbf{R}_{i j}\right) \\
& \times\left\langle\mathrm{e}_{j}\right\rangle_{j}\left(\mathbf{R}_{j}\right) g\left(R_{i j}\right) \mathrm{d}^{3} \mathbf{R}_{j} .
\end{aligned}
$$

To solve the integral equation (70), we look for a solution in the form

$\left\langle\mathrm{e}_{i}\right\rangle_{i}\left(\mathbf{R}_{i}\right)=\sum_{b= \pm} \mathrm{e}^{\mathrm{j} \mathbf{K}_{b} \cdot \mathbf{R}_{i}} \mathrm{e}_{b}$.

Here, $b$ stands for the signs + and - , the vectors $e_{b}$ are unknown and have to be determined, the wave vectors $\mathbf{K}_{b}$ are defined by

$\mathbf{K}_{b}=K \widehat{\mathbf{s}}_{b}=\mathbf{K}_{\perp}+b K_{\mathbf{z}}\left(\mathbf{K}_{\perp}\right) \hat{\mathbf{z}}$,

$K$ is the effective wave number in the particulate medium, and $K_{\mathbf{z}}\left(\mathbf{K}_{\perp}\right)=\sqrt{K^{2}-K_{\perp}^{2}}$. More specifically and as shown in Fig. $3, \mathbf{K}_{+}$ is the upward wave vector in the direction of the transmitted wave $\widehat{\mathbf{s}}_{+}=\widehat{\mathbf{s}}_{\mathrm{T}}=\widehat{\mathbf{s}}_{\mathrm{T}}\left(\theta_{\mathrm{T}}, \varphi_{\mathrm{T}}\right)$, i.e.,

$\mathbf{K}_{+}=\mathbf{K}_{+}\left(\theta_{\mathrm{T}}, \varphi_{\mathrm{T}}\right)=K \widehat{\mathbf{S}}_{\mathrm{T}}=\mathbf{K}_{\perp}+K_{\mathrm{z}}\left(\mathbf{K}_{\perp}\right) \hat{\mathbf{z}}$,

while $\mathbf{K}_{-}$is the downward wave vector in the direction of the transmitted wave reflected by the upper boundary $\widehat{\mathbf{s}}_{-}=\widehat{\mathbf{s}}_{\mathrm{TR}}=$ $\widehat{\mathbf{s}}_{\mathrm{TR}}\left(\theta_{\mathrm{TR}}, \varphi_{\mathrm{T}}\right)$ with $\theta_{\mathrm{TR}}=\pi-\theta_{\mathrm{T}}$, i.e.

$\mathbf{K}_{-}=\mathbf{K}_{-}\left(\theta_{\mathrm{TR}}, \varphi_{\mathrm{T}}\right)=K \widehat{\mathbf{s}}_{\mathrm{TR}}=\mathbf{K}_{\perp}-K_{\mathrm{z}}\left(\mathbf{K}_{\perp}\right) \hat{\mathbf{z}}$

Following Fikioris and Waterman [14,17], we make the following assumptions.
1. The representation (71) for $\left\langle e_{i}\right\rangle_{i}\left(\mathbf{R}_{i}\right)$ is valid for all $z_{i}$ in $D$ even in the critical domains $0 \leq z_{i}<2 a$ and $H-2 a<z_{i} \leq H$.

2 . The vectors $e_{b}$ are obtained by solving the integral equation (70) in the noncritical domain $2 a \leq z_{i} \leq H-2 a$; thus the exclusion domain is a complete sphere of radius $2 a$.

The solution obtained under these assumptions is referred to as the zeroth-order solution. Higher-order solutions can be computed by means of the following iteration scheme. The zeroth-order solution $\left\langle e_{j}^{(0)}\right\rangle_{j}\left(\mathbf{R}_{j}\right)$, which is assumed to be also valid in the critical domains, is inserted in the right-hand side of Eq. (70), and the first-order solution $\left\langle e_{i}^{(1)}\right\rangle_{i}\left(\mathbf{R}_{i}\right)$ is evaluated by taking into account that the domain of integration is $D$ with a truncated sphere of radius $2 a$ excluded. It is obvious that the first-order solution $\left\langle e_{i}^{(1)}\right\rangle_{i}\left(\mathbf{R}_{i}\right)$ is changed only in the critical domains $0 \leq z_{i}<2 a$ and $H-2 a<z_{i} \leq H$, i.e.,

$\left\langle\mathrm{e}_{i}^{(1)}\right\rangle_{i}\left(\mathbf{R}_{i}\right)=\left\langle\mathrm{e}_{i}^{(0)}\right\rangle_{i}\left(\mathbf{R}_{i}\right)$ for $2 a \leq z_{i} \leq H-2 a$,

$\left\langle\mathrm{e}_{i}^{(1)}\right\rangle_{i}\left(\mathbf{R}_{i}\right) \neq\left\langle\mathrm{e}_{i}^{(0)}\right\rangle_{i}\left(\mathbf{R}_{i}\right)$ for $0 \leq z_{i}<2 a$ and $H-2 a<z_{i} \leq H$.

The process is then repeated until convergence is achieved. An important observation is that although the first-order solution $\left\langle e_{i}^{(1)}\right\rangle_{i}\left(\mathbf{R}_{i}\right)$ is changed only in the critical domains, the second-order solution $\left\langle e_{i}^{(2)}\right\rangle_{i}\left(\mathbf{R}_{i}\right)$ and the subsequent ones are changed in the whole domain $D$, i.e.,

$\left\langle\mathrm{e}_{i}^{(n)}\right\rangle_{i}\left(\mathbf{R}_{i}\right) \neq\left\langle\mathrm{e}_{i}^{(n-1)}\right\rangle_{i}\left(\mathbf{R}_{i}\right)$ for $0 \leq z_{i} \leq H$ and $n \geq 2$.

As a result, if the iteration scheme converges, the representation (71) for $\left\langle e_{i}\right\rangle_{i}\left(\mathbf{R}_{i}\right)$ is not valid anymore; the dependency on $\mathbf{R}_{i}$ is much more complex. Because the proof of the convergence of this iteration scheme is a very difficult task, we restrict our analysis to the zeroth-order solution.

In the following, we do not indicate explicitly the dependency of $\left\langle e_{i}\right\rangle_{i}$ on $\mathbf{R}_{i}$. Substituting the solution (71) in Eq. (70), we obtain

$$
\begin{aligned}
\sum_{b= \pm} \mathrm{e}^{\mathrm{j} \mathbf{K}_{b} \cdot \mathbf{R}_{i}} \mathrm{e}_{b} & =\mathrm{e}^{\mathrm{j} \mathbf{k}_{1 \mathrm{~s}} \cdot \mathbf{R}_{i}} \mathrm{e}_{0}+n_{0} \sum_{b= \pm}\left[\int_{D-D_{2 a}\left(\mathbf{R}_{i}\right)} \mathcal{T}_{31}^{T}\left(k_{1} \mathbf{R}_{i j}\right)\right. \\
& \left.\times \mathrm{e}^{\mathrm{j} \mathbf{K}_{b} \cdot \mathbf{R}_{j}} g\left(R_{i j}\right) \mathrm{d}^{3} \mathbf{R}_{j}\right] \mathrm{Te}_{b} .
\end{aligned}
$$

To integrate over all positions of particle $j$ we use a local coordinate system with the origin at particle $i$, and set $\mathbf{R}_{j}=\mathbf{R}_{i}+\mathbf{R}_{j i}$, where $\mathbf{R}_{j i}=-\mathbf{R}_{i j}$. The integral in Eq. (75) (apart from the constant vector $\mathrm{Te}_{b}$ ),

$\mathrm{J}^{b}\left(\mathbf{R}_{i}\right)=\int_{D-D_{2 a}\left(\mathbf{R}_{i}\right)} \mathcal{T}_{31}^{T}\left(-k_{1} \mathbf{R}_{j i}\right) \mathrm{e}^{\mathrm{j} \mathbf{K}_{b} \cdot \mathbf{R}_{j}} g\left(R_{j i}\right) \mathrm{d}^{3} \mathbf{R}_{j i}$,

is written as

$\mathrm{J}^{b}\left(\mathbf{R}_{i}\right)=\mathrm{J}_{1}^{b}\left(\mathbf{R}_{i}\right)+\mathrm{J}_{2}^{b}\left(\mathbf{R}_{i}\right)$,

where

$J_{1}^{b}\left(\mathbf{R}_{i}\right)=\int_{D-D_{2 a}\left(\mathbf{R}_{i}\right)} \mathcal{T}_{31}^{T}\left(-k_{1} \mathbf{R}_{j i}\right) \mathrm{e}^{\mathrm{j} \mathbf{K}_{b} \cdot \mathbf{R}_{j}}\left[g\left(R_{j i}\right)-1\right] \mathrm{d}^{3} \mathbf{R}_{j i}$, 
$\mathrm{J}_{2}^{b}\left(\mathbf{R}_{i}\right)=\int_{D-D_{2 a}\left(\mathbf{R}_{i}\right)} \mathcal{T}_{31}^{T}\left(-k_{1} \mathbf{R}_{j i}\right) \mathrm{e}^{\mathrm{j} \mathbf{K}_{b} \cdot \mathbf{R}_{j}} \mathrm{~d}^{3} \mathbf{R}_{j i}$.

Taking into account the representation of the translation matrix (cf. Eqs. (17) and (23))

$$
\begin{aligned}
\mathcal{T}_{31}^{T}\left(-k_{1} \mathbf{R}_{j i}\right) & =\left[\mathcal{T}_{-m n,-m^{\prime} n^{\prime}}^{31}\left(k_{1} \mathbf{R}_{j i}\right)\right] \\
& =\left[\begin{array}{ll}
A_{-m n,-m^{\prime} n^{\prime}}^{3}\left(k_{1} \mathbf{R}_{j i}\right) & B_{-m n,-m^{\prime} n^{\prime}}^{3}\left(k_{1} \mathbf{R}_{j i}\right) \\
B_{-m n,-m^{\prime} n^{\prime}}^{3}\left(k_{1} \mathbf{R}_{j i}\right) & A_{-m n,-m^{\prime} n^{\prime}}^{3}\left(k_{1} \mathbf{R}_{j i}\right)
\end{array}\right]
\end{aligned}
$$

in terms of radiating spherical wave functions (cf. Eqs. (18) and (19))

$$
\begin{aligned}
A_{-m n,-m^{\prime} n^{\prime}}^{3}\left(k_{1} \mathbf{R}_{j i}\right) & =c_{n n^{\prime}} \sum_{n^{\prime \prime}} \mathrm{j}^{n^{\prime \prime}} a_{1}\left(-m, n\left|-m^{\prime}, n^{\prime}\right| n^{\prime \prime}\right) \\
& \times u_{m^{\prime}-m n^{\prime \prime}}^{3}\left(k_{1} \mathbf{R}_{j i}\right), \\
B_{-m n,-m^{\prime} n^{\prime}}^{3}\left(k_{1} \mathbf{R}_{j i}\right) & =c_{n n^{\prime}} \sum_{n^{\prime \prime}} \mathrm{j}^{n^{\prime \prime}} b_{1}\left(-m, n\left|-m^{\prime}, n^{\prime}\right| n^{\prime \prime}\right) \\
& \times u_{m^{\prime}-m n^{\prime \prime}}^{3}\left(k_{1} \mathbf{R}_{j i}\right),
\end{aligned}
$$

where $c_{n n^{\prime}}$ is given by Eq. (22), we see that the computation of the matrices $J_{1}^{b}\left(\mathbf{R}_{i}\right)$ and $\mathrm{J}_{2}^{b}\left(\mathbf{R}_{i}\right)$ requires the computation of the integrals

$$
\begin{aligned}
I_{1}^{b}\left(\mathbf{R}_{i}\right) & =\mathrm{e}^{\mathrm{j} \mathbf{K}_{b} \cdot \mathbf{R}_{i}} \int_{D-D_{2 a}\left(\mathbf{R}_{i}\right)} u_{m^{\prime}-m n^{\prime \prime}}^{3}\left(k_{1} \mathbf{R}_{j i}\right) \mathrm{e}^{\mathrm{j} \mathbf{K}_{b} \cdot \mathbf{R}_{j i}} \\
& \times\left[g\left(R_{i j}\right)-1\right] \mathrm{d}^{3} \mathbf{R}_{j i}, \\
I_{2}^{b}\left(\mathbf{R}_{i}\right) & =\mathrm{e}^{\mathrm{j} \mathbf{K}_{b} \cdot \mathbf{R}_{i}} \int_{D-D_{2 a}\left(\mathbf{R}_{i}\right)} u_{m^{\prime}-m n^{\prime \prime}}^{3}\left(k_{1} \mathbf{R}_{j i}\right) \mathrm{e}^{\mathrm{j} \mathbf{K}_{b} \cdot \mathbf{R}_{j i}} \mathrm{~d}^{3} \mathbf{R}_{j i} .
\end{aligned}
$$

Computation of $J_{1}^{b}$. To compute $I_{1}^{b}\left(\mathbf{R}_{i}\right)$, and so $J_{1}^{b}\left(\mathbf{R}_{i}\right)$, we take into account that the function $g\left(R_{j i}\right)-1$ quickly decreases with increasing $R_{j i}$, in which case, the domain of integration can be extended to the entire space less a ball of radius $2 a$ around $\mathbf{R}_{i}$. The integral is therefore approximated by

$\int_{D-D_{2 a}\left(\mathbf{R}_{i}\right)} R_{j i}^{2} \mathrm{~d}^{2} \widehat{\mathbf{R}}_{j i} \mathrm{~d} R_{j i} \approx \int_{\mathbb{R}^{3}-D_{2 a}\left(\mathbf{R}_{i}\right)} R_{j i}^{2} \mathrm{~d}^{2} \widehat{\mathbf{R}}_{j i} \mathrm{~d} R_{j i}$.

Using the spherical wave expansion of the plane wave (e.g., Ref. [30])

$\mathrm{e}^{\mathrm{j} \mathbf{K}_{b} \cdot \mathbf{R}_{j i}}=\mathrm{e}^{\mathrm{j} K \widehat{\mathbf{s}}_{b} \cdot \mathbf{R}_{j i}}=\sum_{k l} 2 \mathrm{j}^{l} Y_{-k l}\left(\widehat{\mathbf{s}}_{b}\right) u_{k l}^{1}\left(K \mathbf{R}_{j i}\right)$,

where, for $\widehat{\mathbf{r}}=\widehat{\mathbf{r}}(\theta, \varphi), Y_{k l}(\widehat{\mathbf{r}})=Y_{k l}(\theta, \varphi)$ are spherical harmonics and $u_{k l}^{1}(K \mathbf{r})$ are regular spherical wave functions for wavenumber $K$, we find that $J_{1}^{b}\left(\mathbf{R}_{i}\right)$ can be expressed as

$\mathrm{J}_{1}^{b}\left(\mathbf{R}_{i}\right)=\mathrm{J}_{1 a}^{b} \mathrm{e}^{\mathrm{j} \mathbf{K}_{b} \cdot \mathbf{R}_{i}}$,

where the block-matrix components of the matrix $\mathrm{J}_{1 a}^{b}$,

$J_{1 a}^{b}=\left[\begin{array}{ll}\left(J_{1 a}^{b}\right)_{-m n,-m^{\prime} n^{\prime}}^{11} & \left(J_{1 a}^{b}\right)_{-m n,-m^{\prime} n^{\prime}}^{12} \\ \left(J_{1 a}^{b}\right)_{-m n,-m^{\prime} n^{\prime}}^{21} & \left(J_{1 a}^{b}\right)_{-m n,-m^{\prime} n^{\prime}}^{22}\end{array}\right]$,

are

$$
\begin{aligned}
\left(J_{1 a}^{b}\right)_{-m n,-m^{\prime} n^{\prime}}^{11} & =\left(J_{1 a}^{b}\right)_{-m n,-m^{\prime} n^{\prime}}^{22} \\
& =4 \pi c_{n n^{\prime}} \sum_{n^{\prime \prime}}(-1)^{n^{\prime \prime}} a_{1}\left(-m, n\left|-m^{\prime}, n^{\prime}\right| n^{\prime \prime}\right) \\
& \times Y_{m^{\prime}-m, n^{\prime \prime}}\left(\widehat{\mathbf{s}}_{b}\right) F_{n^{\prime \prime}}\left(k_{1}, K, a\right),
\end{aligned}
$$

$\left(J_{1 a}^{b}\right)_{-m n,-m^{\prime} n^{\prime}}^{12}=\left(J_{1 a}^{b}\right)_{-m n,-m^{\prime} n^{\prime}}^{21}$

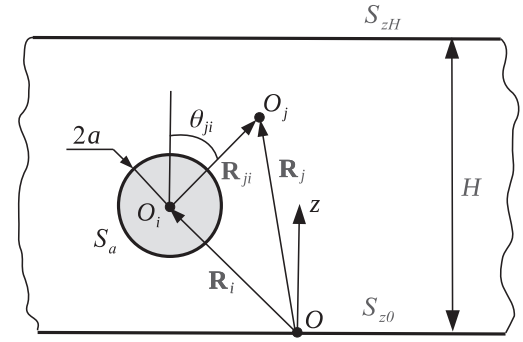

Fig. 4. The integration surfaces $S_{a}, S_{z 0}$, and $S_{z H}$ for computing the integral $I_{2}^{b}\left(\mathbf{R}_{i}\right)$.

$$
\begin{aligned}
& =4 \pi c_{n n^{\prime}} \sum_{n^{\prime \prime}}(-1)^{n^{\prime \prime}} b_{1}\left(-m, n\left|-m^{\prime}, n^{\prime}\right| n^{\prime \prime}\right) \\
& \times Y_{m^{\prime}-m, n^{\prime \prime}}\left(\widehat{\mathbf{s}}_{b}\right) F_{n^{\prime \prime}}\left(k_{1}, K, a\right),
\end{aligned}
$$

and

$$
F_{n^{\prime \prime}}\left(k_{1}, K, a\right)=\int_{2 a}^{\infty}[g(r)-1] h_{n^{\prime \prime}}\left(k_{1} r\right) j_{n^{\prime \prime}}(K r) r^{2} \mathrm{~d} r .
$$

Computation of $\mathrm{J}_{2}^{b}$. To compute $I_{2}^{b}\left(\mathbf{R}_{i}\right)$, the volume integral over $D-D_{2 a}\left(\mathbf{R}_{i}\right)$ is transformed into three surface integrals by means of the scalar Green's theorem of the second kind. For the scalar functions $f=\exp \left(\mathrm{j} \mathbf{K}_{b} \cdot \mathbf{R}_{j i}\right)$ and $g=u_{m^{\prime}-m n^{\prime \prime}}^{3}\left(k_{1} \mathbf{R}_{j i}\right)$ satisfying the scalar wave equation with wavenumbers $K$ and $k_{1}$, respectively, the Green's theorem yields

$\int_{D-D_{2 a}\left(\mathbf{R}_{i}\right)} f g \mathrm{~d} V=\frac{1}{K^{2}-k_{1}^{2}} \oint_{S}\left(f \frac{\partial g}{\partial \widehat{\mathbf{n}}}-g \frac{\partial f}{\partial \widehat{\mathbf{n}}}\right) \mathrm{d} S$,

where, neglecting the vertical boundaries at infinity, the surface $S$ is the union of a sphere of radius $2 a$ centered at $\mathbf{R}_{i}$ and the boundary planes $z_{j}=0$ and $z_{j}=H$, that is, $S=$ $S_{a} \cup S_{\mathrm{z} 0} \cup S_{\mathrm{zH}}$ (see Fig. 4). To compute the integral over $S_{a}$ we use the expansion (86), while for computing the integrals over $S_{z 0}$ and $S_{z H}$ we invoke the integral representation of the radiating spherical waves in terms of plane waves. More specifically, for $z_{i} \geq 2 a$ and in the plane $z_{j}=0$, we use [31]

$u_{m n}^{3}\left(k_{1} \mathbf{R}_{j i}\right)=\frac{1}{2 \pi \mathrm{j}^{n}} \int Y_{m n}\left(\widehat{\mathbf{k}}^{-}\right) \mathrm{e}^{\mathrm{j} \mathbf{k}_{\perp} \cdot \mathbf{R}_{j \perp}} \mathrm{e}^{\mathrm{j} k_{1 \mathrm{z}}\left(\mathbf{k}_{\perp}\right) z_{i}} \frac{\mathrm{d}^{2} \mathbf{k}_{\perp}}{k_{1} k_{1 \mathrm{z}}\left(\mathbf{k}_{\perp}\right)}$,

and for $z_{i} \leq H-2 a$ and in the plane $z_{j}=H$, we use

$$
\begin{aligned}
u_{m n}^{3}\left(k_{1} \mathbf{R}_{j i}\right)= & \frac{1}{2 \pi \mathrm{j}^{n}} \int Y_{m n}\left(\widehat{\mathbf{k}}^{+}\right) \mathrm{e}^{\mathrm{j} \mathbf{k}_{\perp} \cdot \mathbf{R}_{j i \perp}} \mathrm{e}^{\mathrm{j} k_{1 z}\left(\mathbf{k}_{\perp}\right)\left(H-z_{i}\right)} \\
& \times \frac{\mathrm{d}^{2} \mathbf{k}_{\perp}}{k_{1} k_{1 \mathrm{z}}\left(\mathbf{k}_{\perp}\right)} .
\end{aligned}
$$

Here,

$\mathbf{k}^{b}=\mathbf{k}^{b}\left(\mathbf{k}_{\perp}\right)=\mathbf{k}_{\perp}+b k_{1 \mathrm{z}}\left(\mathbf{k}_{\perp}\right) \widehat{\mathbf{z}}, \quad b= \pm$,

$k_{1 \mathrm{z}}\left(\mathbf{k}_{\perp}\right)=\sqrt{k_{1}^{2}-k_{\perp}^{2}}$, and in general for $\mathbf{R}_{i}=\mathbf{R}_{i \perp}+z_{i} \widehat{\mathbf{z}}$ and $\mathbf{R}_{j}=\mathbf{R}_{j \perp}+z_{j} \widehat{\mathbf{z}}$, we have $\mathbf{R}_{j i}=\mathbf{R}_{j i \perp}+\left(z_{j}-z_{i}\right) \widehat{\mathbf{z}}$. Performing the calculations and assuming that the results are also valid in the critical domains, we end up with

$$
\begin{aligned}
J_{2}^{b}\left(\mathbf{R}_{i}\right) & =J_{2 a}^{b} \mathrm{e}^{\mathrm{j} \mathbf{K}_{b} \cdot \mathbf{R}_{i}}+J_{2 z 0}^{b} \mathrm{e}^{\mathrm{j} \mathbf{K}_{\perp} \cdot \mathbf{R}_{i \perp}} \mathrm{e}^{\mathrm{j} k_{1 \mathbf{z}}\left(\mathbf{K}_{\perp}\right) z_{i}} \\
& +J_{2 z \mathrm{H}}^{b} \mathrm{e}^{\mathrm{j} \mathbf{K}_{\perp} \cdot \mathbf{R}_{i \perp}} \mathrm{e}^{-\mathrm{j} k_{1 \mathrm{z}}\left(\mathbf{K}_{\perp}\right) z_{i}} \mathrm{e}^{\mathrm{j}\left[b K_{\mathbf{z}}\left(\mathbf{K}_{\perp}\right)+k_{1 \mathrm{z}}\left(\mathbf{K}_{\perp}\right)\right] H} .
\end{aligned}
$$

The block-matrix components of the matrix $\mathrm{J}_{2 a}^{b}$,

$$
\mathrm{J}_{2 a}^{b}=\left[\begin{array}{ll}
\left(J_{2 a}^{b}\right)_{-m n,-m^{\prime} n^{\prime}}^{11} & \left(J_{2 a}^{b}\right)_{-m n,-m^{\prime} n^{\prime}}^{12} \\
\left(J_{2 a}^{b}\right)_{-m n,-m^{\prime} n^{\prime}}^{21} & \left(J_{2 a}^{b}\right)_{-m n,-m^{\prime} n^{\prime}}^{22}
\end{array}\right],
$$




$$
\begin{aligned}
& \text { are } \\
& \begin{aligned}
\left(J_{2 a}^{b}\right)_{-m n,-m^{\prime} n^{\prime}}^{11} & =\left(J_{2 a}^{b}\right)_{-m n,-m^{\prime} n^{\prime}}^{22} \\
& =-\frac{16 \pi a}{K^{2}-k_{1}^{2}} c_{n n^{\prime}} \sum_{n^{\prime \prime}}(-1)^{n^{\prime \prime}} \\
& \times a_{1}\left(-m, n\left|-m^{\prime}, n^{\prime}\right| n^{\prime \prime}\right) \\
& \times Y_{m^{\prime}-m, n^{\prime \prime}}\left(\widehat{\mathbf{s}}_{b}\right) G_{n^{\prime \prime}}\left(k_{1}, K, a\right), \\
& =\left(J_{2 a}^{b}\right)_{-m n,-m^{\prime} n^{\prime}}^{21} \\
& =-\frac{16 \pi a}{K^{2}-k_{1}^{2}} c_{n n^{\prime}} \sum_{n^{\prime \prime}}(-1)^{n^{\prime \prime}} \\
& \times b_{1}\left(-m, n\left|-m^{\prime}, n^{\prime}\right| n^{\prime \prime}\right) \\
& \times Y_{m^{\prime}-m, n^{\prime \prime}}\left(\widehat{\mathbf{s}}_{b}\right) G_{n^{\prime \prime}}\left(k_{1}, K, a\right),
\end{aligned}
\end{aligned}
$$

where

$$
\begin{aligned}
G_{n^{\prime \prime}}\left(k_{1}, K, a\right) & =\left(k_{1} a\right) h_{n^{\prime \prime}}^{\prime}\left(2 k_{1} a\right) j_{n^{\prime \prime}}(2 K a) \\
& -(K a) h_{n^{\prime \prime}}\left(2 k_{1} a\right) j_{n^{\prime \prime}}^{\prime}(2 K a) .
\end{aligned}
$$

The block matrix components of the matrix $J_{2 z 0}^{b}$,

$$
\mathrm{J}_{2 \mathrm{z} 0}^{b}=\left[\begin{array}{ll}
\left(J_{2 \mathrm{z} 0}^{b}\right)_{-m n,-m^{\prime} n^{\prime}}^{11} & \left(J_{2 \mathrm{z} 0}^{b}\right)_{-m n,-m^{\prime} n^{\prime}}^{12} \\
\left(J_{2 \mathrm{z} 0}^{b}\right)_{-m n,-m^{\prime} n^{\prime}}^{21} & \left(J_{2 \mathrm{z} 0}^{b}\right)_{-m n,-m^{\prime} n^{\prime}}^{22}
\end{array}\right],
$$

are

$$
\begin{aligned}
\left(J_{2 \mathrm{z} 0}^{b}\right)_{-m n,-m^{\prime} n^{\prime}}^{11} & =\left(J_{2 \mathrm{z} 0}^{b}\right)_{-m n,-m^{\prime} n^{\prime}}^{22} \\
& =2 \pi \mathrm{j} \frac{b K_{\mathrm{z}}\left(\mathbf{K}_{\perp}\right)+k_{1 \mathrm{z}}\left(\mathbf{K}_{\perp}\right)}{k_{1} k_{1 \mathrm{z}}\left(\mathbf{K}_{\perp}\right)\left(K^{2}-k_{1}^{2}\right)} c_{n n^{\prime}} \\
& \times \sum_{n^{\prime \prime}}(-1)^{n^{\prime \prime}} a_{1}\left(-m, n\left|-m^{\prime}, n^{\prime}\right| n^{\prime \prime}\right) \\
& \times Y_{m^{\prime}-m, n^{\prime \prime}}\left(\widehat{\mathbf{k}}^{+}\right),
\end{aligned}
$$

$$
\begin{aligned}
\left(J_{2 \mathrm{z} 0}^{b}\right)_{-m n,-m^{\prime} n^{\prime}}^{12} & =\left(J_{2 \mathrm{z} 0}^{b}\right)_{-m n,-m^{\prime} n^{\prime}}^{21} \\
& =2 \pi \mathrm{j} \frac{b K_{\mathrm{z}}\left(\mathbf{K}_{\perp}\right)+k_{1 \mathrm{z}}\left(\mathbf{K}_{\perp}\right)}{k_{1} k_{1 \mathrm{z}}\left(\mathbf{K}_{\perp}\right)\left(K^{2}-k_{1}^{2}\right)} c_{n n^{\prime}} \\
& \times \sum_{n^{\prime \prime}}(-1)^{n^{\prime \prime}} b_{1}\left(-m, n\left|-m^{\prime}, n^{\prime}\right| n^{\prime \prime}\right) \\
& \times Y_{m^{\prime}-m, n^{\prime \prime}}\left(\widehat{\mathbf{k}}^{+}\right),
\end{aligned}
$$

where hereafter (cf. Eq. (95))

$$
\mathbf{k}^{+}=\mathbf{k}^{+}\left(\mathbf{K}_{\perp}\right)=\mathbf{K}_{\perp}+k_{1 \mathrm{z}}\left(\mathbf{K}_{\perp}\right) \text {. }
$$

Finally, the block matrix components of the matrix $J_{2 z H}^{b}$,

$$
J_{2 \mathrm{z} H}^{b}=\left[\begin{array}{ll}
\left(J_{2 \mathrm{z} H}^{b}\right)_{-m n,-m^{\prime} n^{\prime}}^{11} & \left(J_{2 \mathrm{z} H}^{b}\right)_{-m n,-m^{\prime} n^{\prime}}^{12} \\
\left(J_{2 \mathrm{z} H}^{b}\right)_{-m n,-m^{\prime} n^{\prime}}^{21} & \left(J_{2 \mathrm{z} H}^{b}\right)_{-m n,-m^{\prime} n^{\prime}}^{22}
\end{array}\right],
$$

are

$$
\begin{aligned}
\left(J_{2 \mathrm{z} H}^{b}\right)_{-m n,-m^{\prime} n^{\prime}}^{11} & =\left(J_{2 \mathrm{z} H}^{b}\right)_{-m n,-m^{\prime} n^{\prime}}^{22} \\
& =-2 \pi \mathrm{j} \frac{b K_{\mathrm{z}}\left(\mathbf{K}_{\perp}\right)-k_{1 \mathrm{z}}\left(\mathbf{K}_{\perp}\right)}{k_{1} k_{1 \mathrm{z}}\left(\mathbf{K}_{\perp}\right)\left(K^{2}-k_{1}^{2}\right)} c_{n n^{\prime}} \\
& \times \sum_{n^{\prime \prime}}(-1)^{n^{\prime \prime}} a_{1}\left(-m, n\left|-m^{\prime}, n^{\prime}\right| n^{\prime \prime}\right) \\
& \times Y_{m^{\prime}-m, n^{\prime \prime}}\left(\widehat{\mathbf{k}}^{-}\right), \\
\left(J_{2 \mathrm{z} H}^{b}\right)_{-m n,-m^{\prime} n^{\prime}}^{12} & =\left(J_{2 \mathrm{z} H}^{b}\right)_{-m n,-m^{\prime} n^{\prime}}^{21}
\end{aligned}
$$

$$
\begin{aligned}
& =-2 \pi \mathrm{j} \frac{b K_{z}\left(\mathbf{K}_{\perp}\right)-k_{1 \mathrm{z}}\left(\mathbf{K}_{\perp}\right)}{k_{1} k_{1 \mathrm{z}}\left(\mathbf{K}_{\perp}\right)\left(K^{2}-k_{1}^{2}\right)} c_{n n^{\prime}} \\
& \times \sum_{n^{\prime \prime}}(-1)^{n^{\prime \prime}} b_{1}\left(-m, n\left|-m^{\prime}, n^{\prime}\right| n^{\prime \prime}\right) \\
& \times Y_{m^{\prime}-m, n^{\prime \prime}}\left(\widehat{\mathbf{k}}^{-}\right),
\end{aligned}
$$

where hereafter (cf. Eq. (95))

$\mathbf{k}^{-}=\mathbf{k}^{-}\left(\mathbf{K}_{\perp}\right)=\mathbf{K}_{\perp}-k_{1 z}\left(\mathbf{K}_{\perp}\right)$.

The formulas for $J_{2 z 0}^{b}$ and $J_{2 z H}^{b}$ can be simplified by using the identities

$$
\begin{aligned}
\mathbf{m}_{m^{\prime} n^{\prime}}(\widehat{\mathbf{k}}) \cdot \mathbf{m}_{-m n}(\widehat{\mathbf{k}}) & =c_{n} c_{n^{\prime}}(-1)^{n+n^{\prime}} \sum_{n^{\prime \prime}}(-1)^{n^{\prime \prime}} \\
& \times a_{1}\left(-m, n\left|-m^{\prime}, n^{\prime}\right| n^{\prime \prime}\right) Y_{m^{\prime}-m, n^{\prime \prime}}(\widehat{\mathbf{k}}),
\end{aligned}
$$

$$
\begin{aligned}
\mathbf{m}_{m^{\prime} n^{\prime}}(\widehat{\mathbf{k}}) \cdot \mathbf{n}_{-m n}(\widehat{\mathbf{k}}) & =\mathrm{j} c_{n} c_{n^{\prime}}(-1)^{n+n^{\prime}} \sum_{n^{\prime \prime}}(-1)^{n^{\prime \prime}} \\
& \times b_{1}\left(-m, n\left|-m^{\prime}, n^{\prime}\right| n^{\prime \prime}\right) Y_{m^{\prime}-m, n^{\prime \prime}}(\widehat{\mathbf{k}}),
\end{aligned}
$$

where $\widehat{\mathbf{k}}$ is an arbitrary direction and

$c_{n}=\frac{1}{\sqrt{2 \pi n(n+1)}}$.

Note that the identities (109) and (110) have been derived (i) by expanding the scalar fields $\mathbf{m}_{m^{\prime} n^{\prime}}(\widehat{\mathbf{k}}) \cdot \mathbf{m}_{-m n}(\widehat{\mathbf{k}})$ and $\mathbf{m}_{m^{\prime} n^{\prime}}(\widehat{\mathbf{k}}) \cdot \mathbf{n}_{-m n}(\widehat{\mathbf{k}})$ (for fixed indices $m, m^{\prime}, n$ and $n^{\prime}$ ) in terms of the spherical harmonics $Y_{m^{\prime}-m, n^{\prime \prime}}(\widehat{\mathbf{k}})$, (ii) by using the orthogonality property of the spherical harmonics on the unit sphere, and (iii) by employing the representations (20) and (21). We obtain

$$
\begin{aligned}
\left(J_{2 \mathrm{z} 0}^{b}\right)_{-m n,-m^{\prime} n^{\prime}}^{11} & =\left(J_{2 \mathrm{z} 0}^{b}\right)_{-m n,-m^{\prime} n^{\prime}}^{22}=8 \pi^{2} \mathrm{j}(-\mathrm{j})^{n^{\prime}-n} \\
& \times \frac{b K_{\mathrm{z}}\left(\mathbf{K}_{\perp}\right)+k_{1 \mathrm{z}}\left(\mathbf{K}_{\perp}\right)}{k_{1} k_{1 \mathrm{z}}\left(\mathbf{K}_{\perp}\right)\left(K^{2}-k_{1}^{2}\right)} \mathbf{m}_{m^{\prime} n^{\prime}}\left(\widehat{\mathbf{k}}^{+}\right) \cdot \mathbf{m}_{-m n}\left(\widehat{\mathbf{k}}^{+}\right), \\
\left(J_{2 \mathrm{z} 0}^{b}\right)_{-m n,-m^{\prime} n^{\prime}}^{12} & =\left(J_{2 \mathrm{z} 0}^{b}\right)_{-m n,-m^{\prime} n^{\prime}}^{21}=8 \pi^{2} \mathbf{j}(-\mathrm{j})^{n^{\prime}-n+1} \\
& \times \frac{b K_{\mathrm{z}}\left(\mathbf{K}_{\perp}\right)+k_{1 \mathrm{z}}\left(\mathbf{K}_{\perp}\right)}{k_{1} k_{1 \mathrm{z}}\left(\mathbf{K}_{\perp}\right)\left(K^{2}-k_{1}^{2}\right)} \mathbf{m}_{m^{\prime} n^{\prime}}\left(\widehat{\mathbf{k}}^{+}\right) \cdot \mathbf{n}_{-m n}\left(\widehat{\mathbf{k}}^{+}\right),
\end{aligned}
$$

and

$$
\begin{aligned}
\left(J_{2 \mathrm{z} H}^{b}\right)_{-m n,-m^{\prime} n^{\prime}}^{11} & =\left(J_{2 \mathrm{z} H}^{b}\right)_{-m n,-m^{\prime} n^{\prime}}^{22}=-8 \pi^{2} \mathrm{j}(-\mathbf{j})^{n^{\prime}-n} \\
& \times \frac{b K_{\mathrm{z}}\left(\mathbf{K}_{\perp}\right)-k_{1 \mathrm{z}}\left(\mathbf{K}_{\perp}\right)}{k_{1} k_{1 \mathrm{z}}\left(\mathbf{K}_{\perp}\right)\left(K^{2}-k_{1}^{2}\right)} \mathbf{m}_{m^{\prime} n^{\prime}}\left(\widehat{\mathbf{k}}^{-}\right) \cdot \mathbf{m}_{-m n}\left(\widehat{\mathbf{k}}^{-}\right),
\end{aligned}
$$

$$
\begin{aligned}
\left(J_{2 \mathrm{z} H}^{b}\right)_{-m n,-m^{\prime} n^{\prime}}^{12} & =\left(J_{2 \mathrm{zH}}^{b}\right)_{-m n,-m^{\prime} n^{\prime}}^{21}=-8 \pi^{2} \mathrm{j}(-\mathrm{j})^{n^{\prime}-n+1} \\
& \times \frac{b K_{\mathrm{z}}\left(\mathbf{K}_{\perp}\right)-k_{1 \mathrm{z}}\left(\mathbf{K}_{\perp}\right)}{k_{1} k_{1 \mathrm{z}}\left(\mathbf{K}_{\perp}\right)\left(K^{2}-k_{1}^{2}\right)} \mathbf{m}_{m^{\prime} n^{\prime}}\left(\widehat{\mathbf{k}}^{-}\right) \cdot \mathbf{n}_{-m n}\left(\widehat{\mathbf{k}}^{-}\right) .
\end{aligned}
$$

Collecting all results we express the integral equation (75) as

$$
\begin{aligned}
\sum_{b= \pm} \mathrm{e}^{\mathrm{j} \mathbf{K}_{\perp} \cdot \mathbf{R}_{i \perp}} \mathrm{e}^{\mathrm{j} b K_{\mathbf{z}}\left(\mathbf{K}_{\perp}\right) z_{i}} \mathrm{e}_{b} & =\mathrm{e}^{\mathrm{j} \mathbf{k}_{1 \mathrm{~s} \perp} \cdot \mathbf{R}_{i \perp}} \mathrm{e}^{\mathrm{j} k_{1 \mathrm{z}}\left(\mathbf{k}_{1 \mathrm{~s} \perp}\right) z_{i}} \mathrm{e}_{0} \\
& +\sum_{b= \pm} \mathrm{e}^{\mathrm{j} \mathbf{K}_{\perp} \cdot \mathbf{R}_{\perp \perp}} \mathrm{e}^{\mathrm{j} b K_{\mathrm{z}}\left(\mathbf{K}_{\perp}\right) z_{i}} n_{0}\left(\mathrm{~J}_{1 a}^{b}+\mathrm{J}_{2 a}^{b}\right) T \mathrm{e}_{b}
\end{aligned}
$$




$$
\begin{aligned}
& +\mathrm{e}^{\mathrm{j} \mathbf{K}_{\perp} \cdot \mathbf{R}_{\perp \perp}} \mathrm{e}^{\mathrm{j} k_{1 \mathrm{z}}\left(\mathbf{K}_{\perp}\right) z_{i}} n_{0} \sum_{b= \pm} \mathrm{J}_{2 \mathrm{z} 0}^{b} \mathrm{Te}_{b} \\
& +\mathrm{e}^{\mathrm{j} \mathbf{K}_{\perp} \cdot \mathbf{R}_{i \perp}} \mathrm{e}^{-\mathrm{j} k_{1 \mathrm{z}}\left(\mathbf{K}_{\perp}\right) z_{i}} n_{0} \sum_{b= \pm} \mathrm{e}^{\mathrm{j}\left[b K_{\mathrm{z}}\left(\mathbf{K}_{\perp}\right) k_{1 \mathrm{z}}\left(\mathbf{K}_{\perp}\right)\right] H} \\
& \times \mathrm{J}_{2 \mathrm{z} H}^{b} \mathrm{Te}_{b} .
\end{aligned}
$$

In Eq. (116), we first separate the upward and downward propagating waves, and then balance the waves with the wavenumber $k_{1}$ and those with the wavenumber $K$; for the upward propagating waves with the wavenumbers $k_{1}$ and $K$ we obtain, respectively,

$\mathrm{e}^{\mathrm{j} \mathbf{k}_{1 \mathrm{~s} \perp} \cdot \mathbf{R}_{i \perp}} \mathrm{e}^{\mathrm{j} k_{1 \mathrm{z}}\left(\mathbf{k}_{1 \mathbf{s} \perp}\right) z_{i}} e_{0}+\mathrm{e}^{\mathrm{j} \mathbf{K}_{\perp} \cdot \mathbf{R}_{i \perp}} \mathrm{e}^{\mathrm{j} k_{1 \mathrm{z}}\left(\mathbf{K}_{\perp}\right) z_{i}} n_{0} \sum_{b= \pm} \mathrm{J}_{2 \mathrm{z} 0}^{b} \mathrm{Te}_{b}=0$,

$\mathrm{e}_{+}-n_{0}\left(\mathrm{~J}_{1 a}^{+}+\mathrm{J}_{2 a}^{+}\right) \mathrm{Te}_{+}=0$,

while for the downward propagating waves with the wavenumbers $k_{1}$ and $K$ we obtain, respectively,

$\sum_{b= \pm} \mathrm{e}^{\mathrm{j} b K_{\mathrm{z}}\left(\mathbf{K}_{\perp}\right) H} \mathrm{~J}_{2 \mathrm{zH}}^{b} \mathrm{Te}_{b}=0$

$\mathrm{e}_{-}-n_{0}\left(\mathrm{~J}_{1 a}^{-}+\mathrm{J}_{2 a}^{-}\right) \mathrm{Te}_{-}=0$.

From Eq. (117), it follows that

$\mathbf{K}_{\perp}=\mathbf{k}_{1 \mathrm{~s} \perp}$,

$k_{1 \mathrm{z}}\left(\mathbf{K}_{\perp}\right)=k_{1 \mathrm{z}}\left(\mathbf{k}_{1 \mathrm{~s} \perp}\right)=\sqrt{k_{1}^{2}-k_{1 \mathrm{~s} \perp}^{2}}$.

The relation (121), which reflects the translational invariance of the problem, is essentially the Snell law

$K \sin \theta_{\mathrm{T}}=k_{1} \sin \theta_{0}$,

$\varphi_{\mathrm{T}}=\varphi_{0}$

Note also that because of scattering and absorption, the effective wavenumber $K$ is complex; hence, the transmitted angle $\theta_{\mathrm{T}}$ is (slightly) complex.

Hereafter, we introduce the short-hand notation

$K_{\mathrm{z}} \stackrel{\text { def }}{=} K_{\mathrm{z}}\left(\mathbf{k}_{1 \mathrm{~s} \perp}\right)=\sqrt{K^{2}-k_{1 \mathrm{~s} \perp}^{2}}=\sqrt{K^{2}-k_{1}^{2} \sin ^{2} \theta_{0}}$,

and

$k_{1 \mathrm{z}} \stackrel{\text { def }}{=} k_{1 \mathrm{z}}\left(\mathbf{k}_{1 \mathrm{~s} \perp}\right)=\sqrt{k_{1}^{2}-k_{1 \mathrm{~s} \perp}^{2}}=\sqrt{k_{1}^{2}-k_{1}^{2} \sin ^{2} \theta_{0}}$,

and let

$K_{\mathrm{z}}^{\prime}=\operatorname{Re}\left(K_{\mathrm{z}}\right)$ and $K_{\mathrm{z}}^{\prime \prime}=\operatorname{Im}\left(K_{\mathrm{z}}\right)>0$.

The effective wave vectors $\mathbf{K}_{b}$ can then be expressed as

$\mathbf{K}_{b}=\mathbf{k}_{1 \mathrm{~s} \perp}+b K_{\mathrm{z}} \hat{\mathbf{z}}=k_{1} \widehat{\mathbf{s}}+\left(b K_{\mathrm{z}}-k_{1 \mathrm{z}}\right) \hat{\mathbf{z}}$.

From Eq. (121), the vectors $\mathbf{k}^{+}$and $\mathbf{k}^{-}$, defined by Eqs. (104) and (108), respectively, become

$\mathbf{k}^{+}=\mathbf{k}_{1 \mathrm{~s}}=\mathbf{k}_{1 \mathrm{~s} \perp}+k_{1 \mathrm{z}}\left(\mathbf{k}_{1 \mathrm{~s} \perp}\right)=k_{1} \widehat{\mathbf{s}}$

and

$\mathbf{k}^{-}=\mathbf{k}_{1 \mathrm{R}}=\mathbf{k}_{1 \mathrm{~s} \perp}-k_{1 \mathrm{z}}\left(\mathbf{k}_{1 \mathrm{~s} \perp}\right)=k_{1} \widehat{\mathbf{s}}_{\mathrm{R}}$,

where $\widehat{\boldsymbol{s}}_{\mathrm{R}}$ is the specular reflection direction characterized by the spherical angles $\left(\theta_{\mathrm{R}}, \varphi_{0}\right)=\left(\pi-\theta_{0}, \varphi_{0}\right)$. Hence, $\widehat{\mathbf{k}}^{+}=\widehat{\mathbf{s}}$ and $\widehat{\mathbf{k}}^{-}=$ $\widehat{\mathbf{s}}_{R}$, and by means of the designations (125) and (126), we express Eqs. (112)-(113) and (114)-(115) as

$\left(J_{2 \mathrm{z} 0}^{b}\right)_{-m n,-m^{\prime} n^{\prime}}^{11}=\left(J_{2 \mathrm{z} 0}^{b}\right)_{-m n,-m^{\prime} n^{\prime}}^{22}=8 \pi^{2} \mathrm{j}(-\mathrm{j})^{n^{\prime}-n}$

$$
\times \frac{b}{k_{1} k_{1 \mathrm{z}}\left(K_{\mathrm{z}}-b k_{1 \mathrm{z}}\right)} \mathbf{m}_{m^{\prime} n^{\prime}}(\widehat{\mathbf{s}}) \cdot \mathbf{m}_{-m n}(\widehat{\mathbf{s}}),
$$

$$
\begin{aligned}
\left(J_{2 \mathrm{z} 0}^{b}\right)_{-m n,-m^{\prime} n^{\prime}}^{12} & =\left(J_{2 \mathrm{z} 0}^{b}\right)_{-m n,-m^{\prime} n^{\prime}}^{21}=8 \pi^{2} \mathrm{j}(-\mathrm{j})^{n^{\prime}-n+1} \\
& \times \frac{b}{k_{1} k_{1 \mathrm{z}}\left(K_{\mathrm{z}}-b k_{1 \mathrm{z}}\right)} \mathbf{m}_{m^{\prime} n^{\prime}}(\mathbf{s}) \cdot \mathbf{n}_{-m n}(\mathbf{s}),
\end{aligned}
$$

and

$$
\begin{aligned}
\left(J_{\mathrm{z} H}^{b}\right)_{-m n,-m^{\prime} n^{\prime}}^{11} & =\left(J_{2 \mathrm{zH}}^{b}\right)_{-m n,-m^{\prime} n^{\prime}}^{22}=-8 \pi^{2} \mathrm{j}(-\mathrm{j})^{n^{\prime}-n} \\
& \times \frac{b}{k_{1} k_{1 \mathrm{z}}\left(K_{\mathrm{z}}+b k_{1 \mathrm{z}}\right)} \mathbf{m}_{m^{\prime} n^{\prime}}\left(\widehat{\mathbf{S}}_{\mathrm{R}}\right) \cdot \mathbf{m}_{-m n}\left(\widehat{\mathbf{S}}_{\mathrm{R}}\right), \\
\left(J_{\mathrm{z} \mathrm{z} H}^{b}\right)_{-m n,-m^{\prime} n^{\prime}}^{12} & =\left(J_{2 \mathrm{z} H}^{b}\right)_{-m n,-m^{\prime} n^{\prime}}^{21}=-8 \pi^{2} \mathrm{j}(-\mathrm{j})^{n^{\prime}-n+1} \\
& \times \frac{b}{k_{1} k_{1 \mathrm{z}}\left(K_{\mathrm{z}}+b k_{1 \mathrm{z}}\right)} \mathbf{m}_{m^{\prime} n^{\prime}}\left(\widehat{\mathbf{S}}_{\mathrm{R}}\right) \cdot \mathbf{n}_{-m n}\left(\widehat{\mathbf{S}}_{\mathrm{R}}\right),
\end{aligned}
$$

respectively.

Two comments are in order.

1. Eqs. (118) and (120), written in compact form as $\mathrm{e}_{b}=n_{0}\left(\mathrm{~J}_{1 a}^{b}+\mathrm{J}_{2 a}^{b}\right) \mathrm{Te}_{b}, \quad b= \pm$,

are representations of the generalized Lorenz-Lorentz law and are used for deriving a dispersion equation for the effective wavenumber $K$.

2. Eq. (117), written as

$$
\mathrm{e}_{0}+n_{0} \sum_{b= \pm} \mathrm{J}_{2 \mathrm{z} 0}^{b} \mathrm{Te}_{b}=0
$$

together with Eq. (119) represents the generalized EwaldOseen extinction theorem. Essentially, Eq. (136) states that at the lower boundary, the waves produced by the particles situated at the lower and upper boundaries of the medium extinguishes the incident wave. Similarly, Eq. (119) states that at the upper boundary, the wave produced by the particles situated at the lower boundary extinguishes the wave produced by the particles situated at the upper boundary.

For the $\eta$-polarized incident field

$\mathbf{E}_{0 \eta}(\mathbf{r})=\widehat{\boldsymbol{\eta}}(\widehat{\mathbf{s}}) \mathrm{e}^{\mathrm{j} \mathbf{k}_{1 \mathrm{~s} \perp} \cdot \mathbf{r}} \mathrm{e}^{\mathrm{j} k_{1 \mathrm{z}} z}$,

where $\eta=\theta, \varphi$, we consider instead of Eq. (39) the polarizationdependent system of equations (43). The integral equation (70) then transforms into

$\left\langle\mathrm{e}_{i \eta}\right\rangle_{i}=\mathrm{e}^{\mathrm{j} \mathbf{k}_{1 \mathrm{~s}} \cdot \mathbf{R}_{i}} \mathrm{e}_{0 \eta}+n_{0} \int_{D-D_{2 a}\left(\mathbf{R}_{i}\right)} \mathrm{Q}\left(k_{1} \mathbf{R}_{i j}\right)\left\langle\mathrm{e}_{j \eta}\right\rangle_{j} g\left(R_{i j}\right) \mathrm{d}^{3} \mathbf{R}_{j}$,

and its solution is sought in the form

$\left\langle\mathrm{e}_{i \eta}\right\rangle_{i}=\sum_{b= \pm} \mathrm{e}^{\mathrm{j} \mathbf{K}_{b} \cdot \mathbf{R}_{i}} \mathrm{e}_{b \eta}$.

From Eq. (41), i.e., $\mathrm{e}_{0}=\mathcal{E}_{0 \theta} \mathrm{e}_{0 \theta}+\mathcal{E}_{0 \varphi} \mathrm{e}_{0 \varphi}$, and the linearity of the integral equation (138), we obtain

$\left\langle\mathrm{e}_{i}\right\rangle_{i}=\mathcal{E}_{0 \theta}\left\langle\mathrm{e}_{i \theta}\right\rangle_{i}+\mathcal{E}_{0 \varphi}\left\langle\mathrm{e}_{i \varphi}\right\rangle_{i}$,

and so,

$\mathrm{e}_{b}=\mathcal{E}_{0 \theta} \mathrm{e}_{b \theta}+\mathcal{E}_{0 \varphi} \mathrm{e}_{b \varphi}$.

Thus, the case of an incident wave of arbitrary polarization can be calculated by linearly combining the cases of $\theta$ - and $\varphi$-polarized incidence.

Taking into account that the incident field coefficients, concatenated in the vector

$\mathrm{e}_{0 \eta}=\left[\begin{array}{l}e_{0 \eta m n}^{1} \\ e_{0 \eta m n}^{2}\end{array}\right]$ 
are given by (cf. Eqs. (10) and (42))

$e_{0 \eta m n}^{1}=4 \pi \mathrm{j}^{n} \widehat{\boldsymbol{\eta}}(\widehat{\mathbf{s}}) \cdot \mathbf{m}_{-m n}(\widehat{\mathbf{s}})$,

$e_{0 \eta m n}^{2}=-4 \pi \mathrm{j}^{n+1} \widehat{\boldsymbol{\eta}}(\widehat{\mathbf{s}}) \cdot \mathbf{n}_{-m n}(\widehat{\mathbf{s}})$,

we assume a similar form representation for the components of the vector

$\mathrm{e}_{b \eta}=\left[\begin{array}{l}e_{b \eta m n}^{1} \\ e_{b \eta m n}^{2}\end{array}\right]$,

that is,

$e_{b \eta m n}^{1}=4 \pi \mathbf{j}^{n} \widehat{\boldsymbol{\eta}}\left(\widehat{\mathbf{s}}_{b}\right) \cdot \mathbf{m}_{-m n}\left(\widehat{\mathbf{s}}_{b}\right) x_{b \eta n}^{1}$,

$e_{b \eta m n}^{2}=-4 \pi \mathbf{j}^{n+1} \widehat{\boldsymbol{\eta}}\left(\widehat{\mathbf{s}}_{b}\right) \cdot \mathbf{n}_{-m n}\left(\widehat{\mathbf{s}}_{b}\right) x_{b \eta n}^{2}$,

where $\widehat{\mathbf{s}}_{+}=\widehat{\mathbf{s}}_{\mathrm{T}}$ and $\widehat{\mathbf{s}}_{-}=\widehat{\mathbf{s}}_{\mathrm{TR}}$ with $\left(\theta_{\mathrm{TR}}, \varphi_{0}\right)=\left(\pi-\theta_{\mathrm{T}}, \varphi_{0}\right)$. In Eqs. (146) and (147), we suppose that for spherically symmetric particles, the coefficients $x_{b \eta n}^{1}$ and $x_{b \eta n}^{2}$ do not depend on the azimuthal index $m$.

\subsection{The generalized Lorenz-Lorentz law}

For a $\eta$-polarized incidence and a specified $\operatorname{sign} b(b=+$ or $b=$ - ), the homogeneous system of equations (135) written as

$\mathrm{e}_{b \eta}=n_{0}\left(\mathrm{~J}_{1 a}^{b}+\mathrm{J}_{2 a}^{b}\right) \mathrm{Te}_{b \eta}$,

has a nontrivial solution if the corresponding determinant vanishes. Equating the determinant to zero gives the dispersion equation for the effective wavenumber $K$.

In explicit form, the homogeneous system of equations (148) is

$$
\begin{aligned}
\widehat{\boldsymbol{\eta}}\left(\widehat{\mathbf{s}}_{b}\right) & \cdot \mathbf{m}_{-m n}\left(\widehat{\mathbf{s}}_{b}\right) x_{b \eta n}^{1} \\
= & 4 \pi n_{0} \sum_{m^{\prime} n^{\prime}} \sum_{n^{\prime \prime}} c_{n n^{\prime}} \mathrm{j}^{n^{\prime}-n}(-1)^{n^{\prime \prime}} H_{n^{\prime \prime}}\left(k_{1}, K, a\right) \\
& \times\left\{a_{1}\left(-m, n\left|-m^{\prime}, n^{\prime}\right| n^{\prime \prime}\right) Y_{m^{\prime}-m, n^{\prime \prime}}\left(\widehat{\mathbf{s}}_{b}\right)\right. \\
& \times\left[\widehat{\boldsymbol{\eta}}\left(\widehat{\mathbf{s}}_{b}\right) \cdot \mathbf{m}_{-m^{\prime} n^{\prime}}\left(\widehat{\mathbf{s}}_{b}\right)\right] T_{n^{\prime}}^{1} x_{b \eta n^{\prime}}^{1} \\
& -\mathrm{j} b_{1}\left(-m, n\left|-m^{\prime}, n^{\prime}\right| n^{\prime \prime}\right) Y_{m^{\prime}-m, n^{\prime \prime}}\left(\widehat{\mathbf{s}}_{b}\right) \\
& \left.\times\left[\widehat{\boldsymbol{\eta}}\left(\widehat{\mathbf{s}}_{b}\right) \cdot \mathbf{n}_{-m^{\prime} n^{\prime}}\left(\widehat{\mathbf{s}}_{b}\right)\right] T_{n^{\prime}}^{2} x_{b \eta n^{\prime}}^{2}\right\}
\end{aligned}
$$

and

$$
\begin{aligned}
\widehat{\boldsymbol{\eta}}\left(\widehat{\mathbf{s}}_{b}\right) & \cdot \mathbf{n}_{-m n}\left(\widehat{\mathbf{s}}_{b}\right) x_{b \eta n}^{2} \\
= & 4 \pi n_{0} \sum_{m^{\prime} n^{\prime}} c_{m n^{\prime}} \mathrm{j}^{n^{\prime}-n}(-1)^{n^{\prime \prime}} H_{n^{\prime \prime}}\left(k_{1}, K, a\right) \\
& \times\left\{\mathrm{j} b_{1}\left(-m, n\left|-m^{\prime}, n^{\prime}\right| n^{\prime \prime}\right) Y_{m^{\prime}-m, n^{\prime \prime}}\left(\widehat{\mathbf{s}}_{b}\right)\right. \\
& \times\left[\widehat{\boldsymbol{\eta}}\left(\widehat{\mathbf{s}}_{b}\right) \cdot \mathbf{m}_{-m^{\prime} n^{\prime}}\left(\widehat{\mathbf{s}}_{b}\right)\right] T_{n^{\prime}}^{1} x_{b \eta n^{\prime}}^{1} \\
& +a_{1}\left(-m, n\left|-m^{\prime}, n^{\prime}\right| n^{\prime \prime}\right) Y_{m^{\prime}-m, n^{\prime \prime}}\left(\widehat{\mathbf{s}}_{b}\right) \\
& \left.\times\left[\widehat{\boldsymbol{\eta}}\left(\widehat{\mathbf{s}}_{b}\right) \cdot \mathbf{n}_{-m^{\prime} n^{\prime}}\left(\widehat{\mathbf{s}}_{b}\right)\right] T_{n^{\prime}}^{2} x_{b \eta n^{\prime}}^{2}\right\},
\end{aligned}
$$

where

$H_{n^{\prime \prime}}\left(k_{1}, K, a\right)=F_{n^{\prime \prime}}\left(k_{1}, K, a\right)-\frac{4 a}{K^{2}-k_{1}^{2}} G_{n^{\prime \prime}}\left(k_{1}, K, a\right)$

and

$\mathrm{T}=\left[T_{n} \delta_{m m^{\prime}} \delta_{n n^{\prime}}\right], \quad\left[T_{n}\right]=\left[\begin{array}{c}T_{n}^{1} \\ T_{n}^{2}\end{array}\right]$

Using the relations

$Y_{m^{\prime}-m, n^{\prime \prime}}(\widehat{\mathbf{k}}) \mathbf{m}_{-m^{\prime} n^{\prime}}(\widehat{\mathbf{k}})=\sum_{p} \frac{(-1)^{p+n^{\prime}+n^{\prime \prime}}}{\sqrt{p n^{\prime}(p+1)\left(n^{\prime}+1\right)}}$

$$
\times\left[a_{1}\left(m, p\left|m^{\prime}, n^{\prime}\right| n^{\prime \prime}\right) \mathbf{m}_{-m p}(\widehat{\mathbf{k}})+\mathrm{j} b_{1}\left(m, p\left|m^{\prime}, n^{\prime}\right| n^{\prime \prime}\right) \mathbf{n}_{-m n}(\widehat{\mathbf{k}})\right]
$$

$$
\begin{aligned}
& Y_{m^{\prime}-m, n^{\prime \prime}}(\widehat{\mathbf{k}}) \mathbf{n}_{-m^{\prime} n^{\prime}}(\widehat{\mathbf{k}}) \\
& =\sum_{p} \frac{(-1)^{p+n^{\prime}+n^{\prime \prime}}}{\sqrt{p n^{\prime}(p+1)\left(n^{\prime}+1\right)}} \\
& \times\left[a_{1}\left(m, p\left|m^{\prime}, n^{\prime}\right| n^{\prime \prime}\right) \mathbf{n}_{-m p}(\widehat{\mathbf{k}})-\mathrm{j} b_{1}\left(m, p\left|m^{\prime}, n^{\prime}\right| n^{\prime \prime}\right) \mathbf{m}_{-m n}(\widehat{\mathbf{k}})\right]
\end{aligned}
$$

which follow from Eqs. (109) and (110), and the summation rules [5]

$$
\begin{aligned}
& \sum_{m^{\prime}} a_{1}\left(-m, n\left|-m^{\prime}, n^{\prime}\right| n^{\prime \prime}\right) a_{1}\left(m, p\left|m^{\prime}, n^{\prime}\right| n^{\prime \prime}\right) \\
& =\delta_{p n} C_{n n^{\prime} n^{\prime \prime}} a_{1}\left(-1, n\left|-1, n^{\prime}\right| n^{\prime \prime}\right),
\end{aligned}
$$

$$
\begin{aligned}
& \sum_{m^{\prime}} b_{1}\left(-m, n\left|-m^{\prime}, n^{\prime}\right| n^{\prime \prime}\right) b_{1}\left(m, p\left|m^{\prime}, n^{\prime}\right| n^{\prime \prime}\right) \\
& =-\delta_{p n} C_{n n^{\prime} n^{\prime \prime}} b_{1}\left(-1, n\left|-1, n^{\prime}\right| n^{\prime \prime}\right),
\end{aligned}
$$

and

$\sum_{m^{\prime}} a_{1}\left(-m, n\left|-m^{\prime}, n^{\prime}\right| n^{\prime \prime}\right) b_{1}\left(m, p\left|m^{\prime}, n^{\prime}\right| n^{\prime \prime}\right)=0$,

where

$C_{n n^{\prime} n^{\prime \prime}}=(-1)^{n+n^{\prime}+n^{\prime \prime}} \sqrt{n n^{\prime}(n+1)\left(n^{\prime}+1\right)} \sqrt{\frac{2 n^{\prime}+1}{2 n+1}} \sqrt{\frac{2 n^{\prime \prime}+1}{2}}$,

we obtain

$x_{b \eta n}^{1}=\sum_{n^{\prime}}\left(A_{n n^{\prime}} T_{n^{\prime}}^{1} x_{b \eta n^{\prime}}^{1}+B_{n n^{\prime}} T_{n^{\prime}}^{2} x_{b \eta n^{\prime}}^{1}\right)$,
$x_{b \eta n}^{2}=\sum_{n^{\prime}}\left(B_{n n^{\prime}} T_{n^{\prime}}^{1} x_{b \eta n^{\prime}}^{1}+A_{n n^{\prime}} T_{n^{\prime}}^{2} x_{b \eta n^{\prime}}^{2}\right)$,

where

$$
\begin{aligned}
A_{n n^{\prime}} & =4 \pi n_{0} \sum_{n^{\prime \prime}} c_{n n^{\prime}} \mathrm{j}^{n^{\prime}-n} \sqrt{\frac{2 n^{\prime}+1}{2 n+1}}(-1)^{n^{\prime \prime}} \sqrt{\frac{2 n^{\prime \prime}+1}{2}} \\
& \times H_{n^{\prime \prime}}\left(k_{1}, K, a\right) a_{1}\left(-1, n\left|-1, n^{\prime}\right| n^{\prime \prime}\right), \\
B_{n n^{\prime}} & =4 \pi n_{0} \sum_{n^{\prime \prime}} c_{n n^{\prime}} \mathrm{j}^{n^{\prime}-n} \sqrt{\frac{2 n^{\prime}+1}{2 n+1}}(-1)^{n^{\prime \prime}} \sqrt{\frac{2 n^{\prime \prime}+1}{2}} \\
& \times H_{n^{\prime \prime}}\left(k_{1}, K, a\right) b_{1}\left(-1, n\left|-1, n^{\prime}\right| n^{\prime \prime}\right) .
\end{aligned}
$$

The dispersion equation is then

$\operatorname{det}\left(\begin{array}{cc}\delta_{n n^{\prime}}-A_{n n^{\prime}} T_{n^{\prime}}^{1} & -B_{n n^{\prime}} T_{n^{\prime}}^{2} \\ -B_{n n^{\prime}} T_{n^{\prime}}^{1} & \delta_{n n^{\prime}}-A_{n n^{\prime}} T_{n^{\prime}}^{2}\end{array}\right)=0$

where $n, n^{\prime}=1, \ldots, N_{\text {rank }}$ and $N_{\text {rank }}$ is the maximum expansion order in the expansions (159)-(162).

It is obvious that the dispersion equation is the same for $b=+$ and $b=-$, and that it is polarization independent. Moreover, the two homogeneous systems of equations (148), corresponding to $b=+$ and $b=-$, reduce to a single homogeneous system of equations, i.e., Eqs. (159) and (160), which is identical to that of a semiinfinite discrete random medium at normal incidence (when only the azimuthal mode $m=1$ is involved in the calculation). Thus, the dispersion equation is also direction independent, and the effective wavenumber of a discrete random layer with non-scattering 


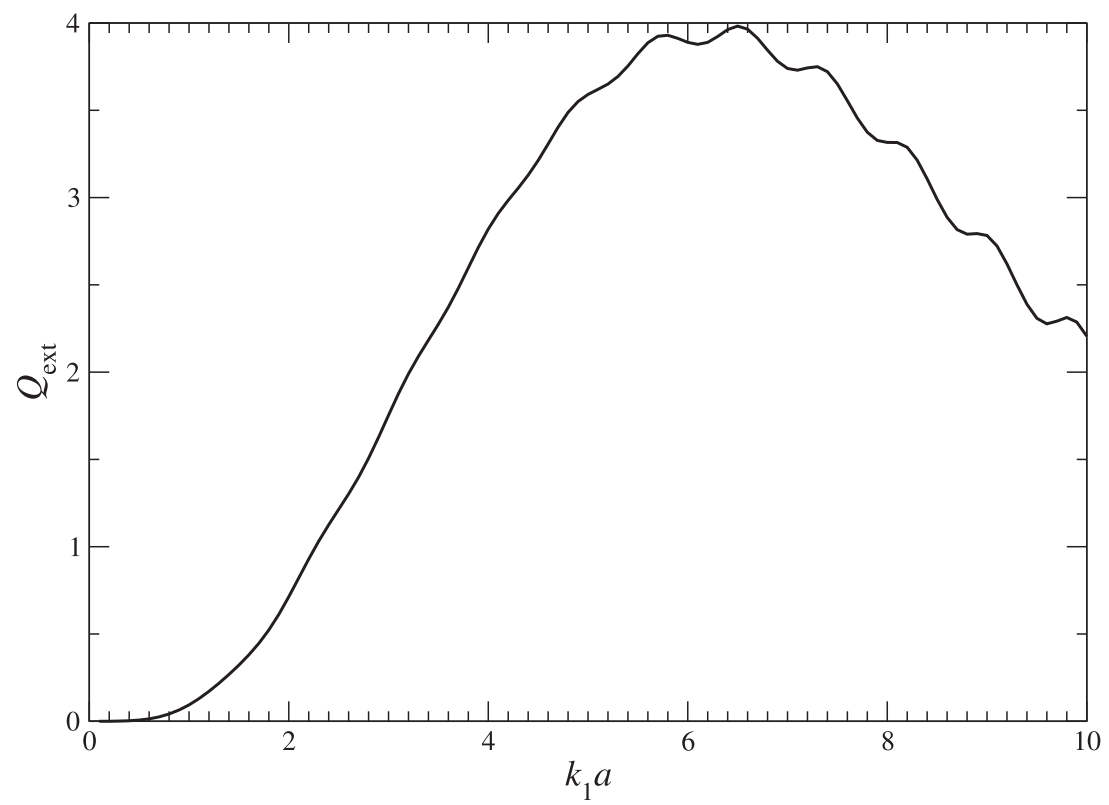

Fig. 5. Extinction efficiency $Q_{\text {ext }}$ versus size parameter $k_{1} a$ for a spherical particle with the relative refractive index $\mathrm{m}=1.33$.
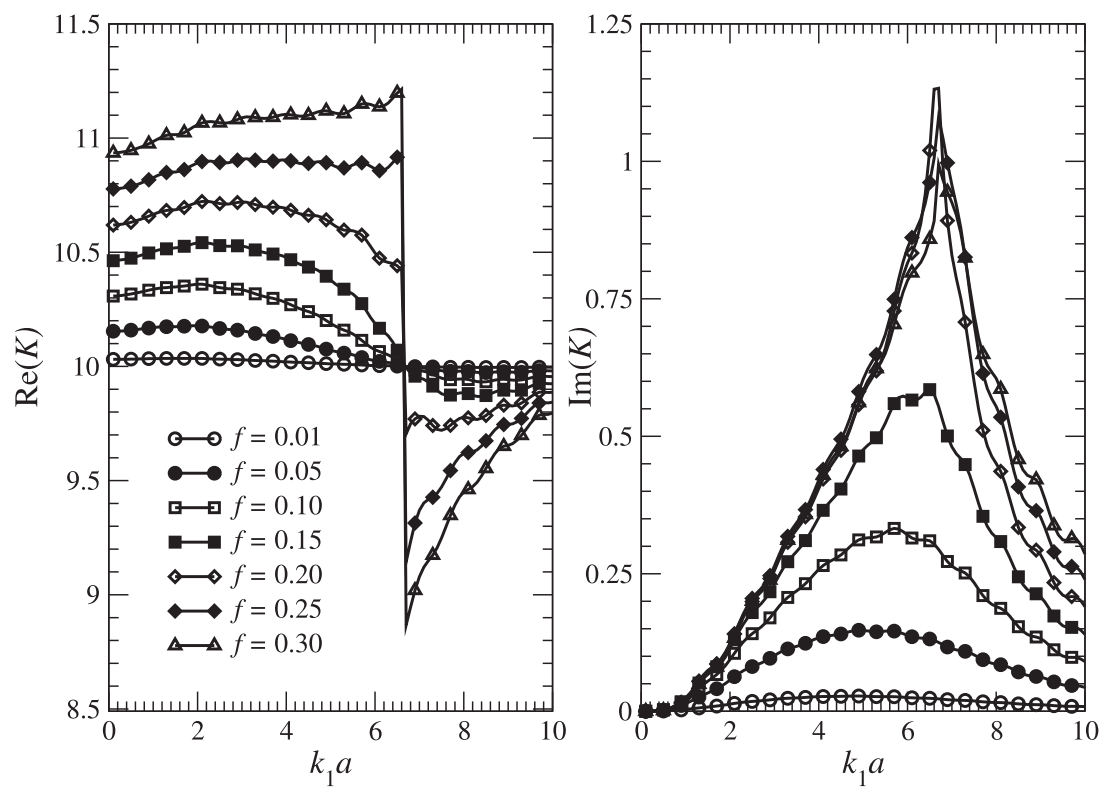

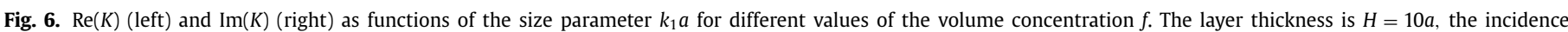

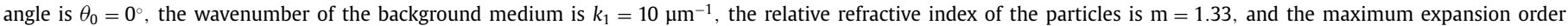
is $N_{\text {rank }}=15$.

boundaries coincides with the effective wavenumber of a semiinfinite discrete random medium.

As examples, we illustrate in Fig. 5 the dependency of the extinction efficiency $Q_{\text {ext }}$ on the size parameter $k_{1} a$ for a spherical particle with the relative refractive index $\mathrm{m}=1.33$ (rain drops at optical frequencies) and in Fig. 6 the real and imaginary parts of the effective wavenumber $\operatorname{Re}(K)$ and $\operatorname{Im}(K)$, respectively, as functions of the size parameter $k_{1} a$ and for different values of the volume concentration $f$. From Fig. 5 we observe that with increasing the size parameter, $Q_{\text {ext }}$ first grows, reaches a maximum at $x_{0}=k_{1} a_{0} \approx 6$, and then decreases; this means that the particles scatter less. As a result, from Fig. 6 we see that

1. for $k_{1} a \leq x_{0}, \operatorname{Re}(K)>k_{1}$ and $\operatorname{Re}(K)$ increases with the volume concentration $f$, while for $k_{1} a>x_{0}, \operatorname{Re}(K)<k_{1}$ and $\operatorname{Re}(K)$ decreases with the volume concentration $f$;
2. $\operatorname{Im}(K)$ as a function of the size parameter $k_{1} a$ has a maximum which moves toward $x_{0}$ when the volume concentration $f$ increases.

Fig. 7 shows that for a spherical particle with the size parameter $k_{1} a=0.5, \operatorname{Im}(K)$ as a function of the volume concentration $f$ has a maximum. This result can be explained by the fact that the particulate medium becomes essentially continuous in terms of its optical properties when the volume concentration is sufficiently high. Consequently, $\operatorname{Im}(K)$ is mostly determined by the absorption of the particles, and for non-absorbing particles, $\operatorname{Im}(K)$ may tend to zero.

We conclude this section by particularizing the above results in the low frequency limit when the size of the particles is much smaller than the wavelength. In this regime, it is sufficient to take only the lowest-order coefficients in the field expansions 


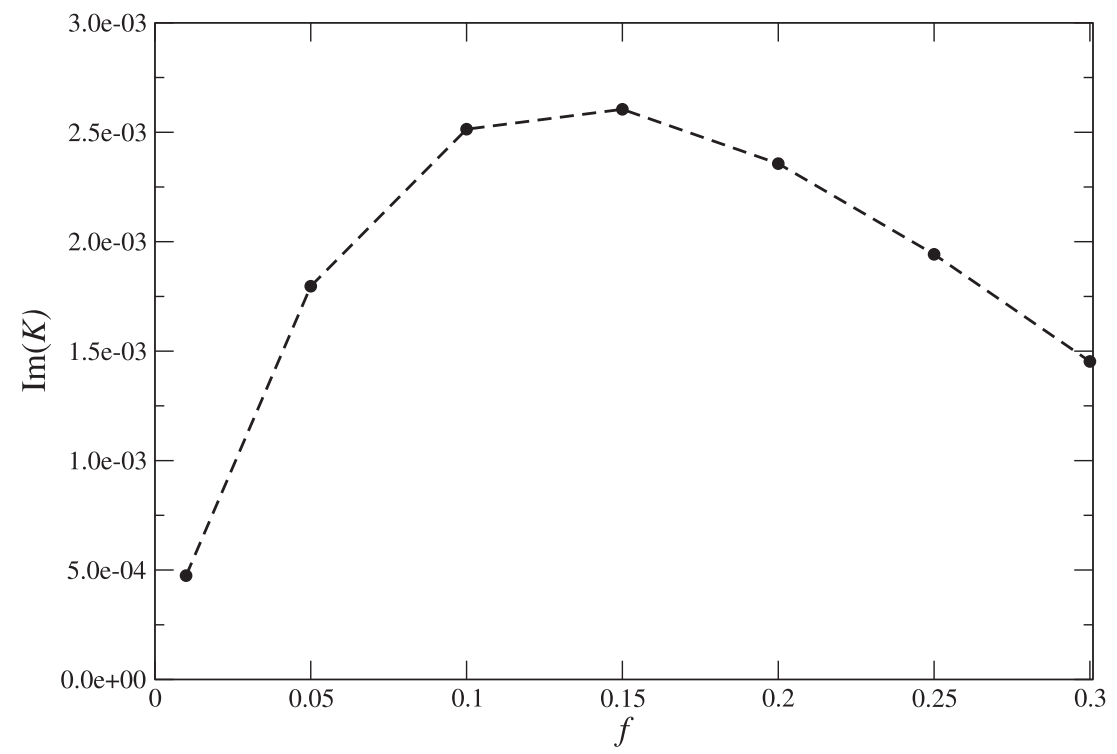

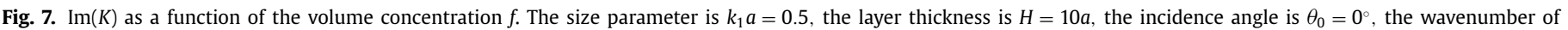
the background medium is $k_{1}=10 \mu \mathrm{m}^{-1}$, the relative refractive index of the particle is $\mathrm{m}=1.33$, and the maximum expansion order is $N_{\text {rank }}=15$.

$\left(N_{\text {rank }}=1\right)$, so that the elements of the T matrix are

$T_{1}^{1}=\frac{\mathrm{j}}{45}\left(\mathrm{~m}^{2}-1\right) x^{5}+O\left(x^{7}\right)$,

$T_{1}^{2}=\frac{2 j}{3} \frac{m^{2}-1}{m^{2}+2} x^{3}+\frac{2 j}{5} \frac{\left(m^{2}-1\right)\left(m^{2}-2\right)}{\left(m^{2}+2\right)^{2}} x^{5}+O\left(x^{6}\right)$,

where $x=k_{1} a$ is the size parameter. In Eqs. (161) and (162) we proceed analogously; considering the leading terms in the expansions of the spherical Bessel and Hankel functions, we obtain

$A_{11}=a_{0}+\frac{a_{2}}{2}, B_{11}=\frac{3 a_{1}}{2}$,

where

$a_{0}=\frac{3 \mathrm{j} f k_{1}^{2}}{\left(k_{1}^{2}-K^{2}\right) x^{3}}+\frac{(1-f)^{4}}{(1+2 f)^{2}}$,

$a_{1}=\frac{3 \mathrm{j} f k_{1} K}{\left(k_{1}^{2}-K^{2}\right) x^{3}}$,

$a_{2}=\frac{3 \mathrm{j} f K^{2}}{\left(k^{2}-K^{2}\right) x^{3}}$.

As a result, the dispersion equation (163) takes the form

$\left(1-A_{11} T_{1}^{1}\right)\left(1-A_{11} T_{1}^{2}\right)-\left(B_{11}\right)^{2} T_{1}^{1} T_{1}^{2}=0$.

Further, neglecting $T_{1}^{1}$ yields $1-A_{11} T_{1}^{2}=0$ and consequently,

$K^{2}=k_{1}^{2} \frac{2 \chi+6 \mathrm{j} f}{2 \chi-3 \mathrm{j} f}$

with

$\chi=x^{3}\left[\frac{(1-f)^{4}}{(1+2 f)^{2}}-\frac{1}{T_{1}^{2}}\right]$

For an ensemble of particles with a small volume concentration $f$, the first term on the right-hand side of Eq. (172) can be neglected; we obtain

$\chi \approx \frac{3 \mathrm{j}}{2} \frac{\mathrm{m}^{2}+2}{\mathrm{~m}^{2}-1}$, and so the Maxwell-Garnet formula

$K^{2}=k_{1}^{2}\left(1+\frac{3 f y}{1-f y}\right) \approx k_{1}^{2}(1+3 f y)$

with $y=\left(m^{2}-1\right) /\left(m^{2}+2\right)$.

\subsection{The generalized Ewald-Oseen extinction theorem}

After computing the effective wavenumber $K$, the components of the vectors $e_{b \eta}$ can be expressed in terms of two arbitrary constants, one for $e_{+}$and the other one for $e_{-\eta}$. These two constants are determined from the two $\eta$-polarized systems of equations of the generalized Ewald-Oseen extinction theorem:

$\mathrm{e}_{0 \eta}+n_{0} \sum_{b= \pm} \mathrm{J}_{2 \mathrm{z} 0}^{b} \mathrm{Te}_{b \eta}=0$

$\sum_{b= \pm} \mathrm{e}^{\mathrm{j} b K_{z} H} J_{2 z H}^{b} \mathrm{Te}_{b \eta}=0$

provided that each system of equations reduces to a single scalar equation. To derive the latter, we use the addition theorem for vector spherical harmonics (see Appendix B), which yields the relations

$\sum_{m}\left[\mathbf{m}_{m n}(\widehat{\mathbf{k}}) \otimes \mathbf{m}_{-m n}\left(\widehat{\mathbf{k}}^{\prime}\right)\right] \cdot \widehat{\boldsymbol{\theta}}\left(\widehat{\mathbf{k}}^{\prime}\right)=\chi_{n} \sqrt{n(n+1)} M_{n}\left(\widehat{\mathbf{k}} \cdot \widehat{\mathbf{k}}^{\prime}\right) \widehat{\boldsymbol{\theta}}(\widehat{\mathbf{k}})$,

$\sum_{m}\left[\mathbf{m}_{m n}(\widehat{\mathbf{k}}) \otimes \mathbf{n}_{-m n}\left(\widehat{\mathbf{k}}^{\prime}\right)\right] \cdot \widehat{\boldsymbol{\theta}}\left(\widehat{\mathbf{k}}^{\prime}\right)=\chi_{n} \sqrt{n(n+1)} N_{n}\left(\widehat{\mathbf{k}} \cdot \widehat{\mathbf{k}^{\prime}}\right) \widehat{\boldsymbol{\varphi}}(\widehat{\mathbf{k}})$,

$\sum_{m}\left[\mathbf{n}_{m n}(\widehat{\mathbf{k}}) \otimes \mathbf{n}_{-m n}\left(\widehat{\mathbf{k}}^{\prime}\right)\right] \cdot \widehat{\boldsymbol{\theta}}\left(\widehat{\mathbf{k}}^{\prime}\right)=-\chi_{n} \sqrt{n(n+1)} N_{n}\left(\widehat{\mathbf{k}} \cdot \widehat{\mathbf{k}} \widehat{\boldsymbol{\theta}}^{\prime} \widehat{\boldsymbol{\theta}}(\widehat{\mathbf{k}})\right.$

for a $\theta$-polarized incidence, and

$\sum_{m}\left[\mathbf{m}_{m n}(\widehat{\mathbf{k}}) \otimes \mathbf{m}_{-m n}\left(\widehat{\mathbf{k}}^{\prime}\right)\right] \cdot \widehat{\boldsymbol{\varphi}}\left(\widehat{\mathbf{k}}^{\prime}\right)=-\chi_{n} \sqrt{n(n+1)} N_{n}\left(\widehat{\mathbf{k}} \cdot \widehat{\mathbf{k}}{ }^{\prime}\right) \widehat{\boldsymbol{\varphi}}(\widehat{\mathbf{k}})$, 
$\sum_{m}\left[\mathbf{m}_{m n}(\widehat{\mathbf{k}}) \otimes \mathbf{n}_{-m n}\left(\widehat{\mathbf{k}}^{\prime}\right)\right] \cdot \widehat{\boldsymbol{\varphi}}\left(\widehat{\mathbf{k}}^{\prime}\right)=-\chi_{n} \sqrt{n(n+1)} M_{n}\left(\widehat{\mathbf{k}} \cdot \widehat{\mathbf{k}}^{\prime}\right) \widehat{\boldsymbol{\theta}}(\widehat{\mathbf{k}})$,

$\sum_{m}\left[\mathbf{n}_{m n}(\widehat{\mathbf{k}}) \otimes \mathbf{n}_{-m n}\left(\widehat{\mathbf{k}}^{\prime}\right)\right] \cdot \widehat{\boldsymbol{\varphi}}\left(\widehat{\mathbf{k}}^{\prime}\right)=\chi_{n} \sqrt{n(n+1)} M_{n}\left(\widehat{\mathbf{k}} \cdot \widehat{\mathbf{k}}^{\prime}\right) \widehat{\boldsymbol{\varphi}}(\widehat{\mathbf{k}})$

for a $\varphi$-polarized incidence. In Eqs. (177)-(181), the coefficient $\chi_{n}$ is given by

$\chi_{n}=\frac{1}{2 \pi n(n+1)} \sqrt{\frac{2 n+1}{2}}$,

while for the directions $\widehat{\mathbf{k}}=\widehat{\mathbf{k}}(\theta, \varphi)$ and $\widehat{\mathbf{k}}^{\prime}=\widehat{\mathbf{k}}^{\prime}\left(\theta^{\prime}, \varphi\right)$, the functions $M_{n}\left(\widehat{\mathbf{k}} \cdot \widehat{\mathbf{k}}^{\prime}\right)$ and $N_{n}\left(\widehat{\mathbf{k}} \cdot \widehat{\mathbf{k}}^{\prime}\right)$ are given respectively by

$M_{n}\left(\widehat{\mathbf{k}} \cdot \widehat{\mathbf{k}}^{\prime}\right)=\pi_{n}^{1}(x)$,

$N_{n}\left(\widehat{\mathbf{k}} \cdot \widehat{\mathbf{k}}^{\prime}\right)=x \pi_{n}^{1}(x)-\sqrt{n(n+1)} P_{n}(x)$,

where $x=\widehat{\mathbf{k}} \cdot \widehat{\mathbf{k}}^{\prime}$ and $\pi_{n}^{1}(x)=P_{n}^{1}(x) / \sqrt{1-x^{2}}$.

Let us analyze Eqs. (175) and (176) in detail.

Eq. (175). Inserting Eqs. (143)-(144) and (146)-(147) together with Eqs. (131)-(132) in Eq. (175), we find that for a $\eta$ polarized incidence,

$$
\begin{aligned}
& \widehat{\boldsymbol{\eta}}(\widehat{\mathbf{s}}) \cdot \mathbf{m}_{-m n}(\widehat{\mathbf{s}}) \\
& =-\mathrm{j} \frac{8 \pi^{2} n_{0}}{k_{1} k_{1 z}} \sum_{b= \pm} \frac{b}{K_{z}-b k_{1 z}} \\
& \times \sum_{m^{\prime} n^{\prime}}\left\{\mathbf{m}_{-m n}(\widehat{\mathbf{s}}) \cdot\left\{\left[\mathbf{m}_{m^{\prime} n^{\prime}}(\widehat{\mathbf{s}}) \otimes \mathbf{m}_{-m^{\prime} n^{\prime}}\left(\widehat{\mathbf{s}}_{b}\right)\right] \cdot \widehat{\boldsymbol{\eta}}\left(\widehat{\mathbf{s}}_{b}\right)\right\} T_{n^{\prime}}^{1} x_{b \eta n^{\prime}}^{1}\right. \\
& \left.-\mathbf{n}_{-m n}(\widehat{\mathbf{s}}) \cdot\left\{\left[\mathbf{m}_{m^{\prime} n^{\prime}}(\widehat{\mathbf{s}}) \otimes \mathbf{n}_{-m^{\prime} n^{\prime}}\left(\widehat{\mathbf{s}}_{b}\right)\right] \cdot \widehat{\boldsymbol{\eta}}\left(\widehat{\mathbf{s}}_{b}\right)\right\} T_{n^{\prime}}^{2} x_{b \eta n^{\prime}}^{2}\right\}
\end{aligned}
$$

and

$$
\begin{aligned}
& \widehat{\boldsymbol{\eta}}(\widehat{\mathbf{s}}) \cdot \mathbf{n}_{-m n}(\widehat{\mathbf{s}}) \\
& =-\mathrm{j} \frac{8 \pi^{2} n_{0}}{k_{1} k_{1 \mathrm{z}}} \sum_{b= \pm} \frac{b}{K_{\mathrm{z}}-b k_{1 \mathrm{z}}} \\
& \times \sum_{m^{\prime} n^{\prime}}\left\{\mathbf{n}_{-m n}(\widehat{\mathbf{s}}) \cdot\left\{\left[\mathbf{m}_{m^{\prime} n^{\prime}}(\widehat{\mathbf{s}}) \otimes \mathbf{m}_{-m^{\prime} n^{\prime}}\left(\widehat{\mathbf{s}}_{b}\right)\right] \cdot \widehat{\boldsymbol{\eta}}\left(\widehat{\mathbf{s}}_{b}\right)\right\} T_{n^{\prime}}^{1} x_{b \eta n^{\prime}}^{1}\right. \\
& \left.+\mathbf{m}_{-m n}(\widehat{\mathbf{s}}) \cdot\left\{\left[\mathbf{m}_{m^{\prime} n^{\prime}}(\widehat{\mathbf{s}}) \otimes \mathbf{n}_{-m^{\prime} n^{\prime}}\left(\widehat{\mathbf{s}}_{b}\right)\right] \cdot \widehat{\boldsymbol{\eta}}\left(\widehat{\mathbf{s}}_{b}\right)\right\} T_{n^{\prime}}^{2} x_{b \eta n^{\prime}}^{2}\right\}
\end{aligned}
$$

Using Eqs. (177), (178), (180), and (181) of the addition theorem for vector spherical harmonics, we get

$$
\begin{aligned}
1 & =-\mathrm{j} \frac{8 \pi^{2} n_{0}}{k_{1} k_{1 \mathrm{z}}} \sum_{b= \pm} \frac{b}{K_{\mathrm{z}}-b k_{1 z}} \sum_{n} \chi_{n} \sqrt{n(n+1)} \\
& \times\left[M_{n}\left(\widehat{\mathbf{s}} \cdot \widehat{\mathbf{s}}_{b}\right) T_{n}^{1} x_{b \theta n}^{1}-N_{n}\left(\mathbf{s} \cdot \widehat{\mathbf{s}}_{b}\right) T_{n}^{2} x_{b \theta n}^{2}\right]
\end{aligned}
$$

for a $\theta$-polarized incidence, and

$$
\begin{aligned}
1 & =\mathrm{j} \frac{8 \pi^{2} n_{0}}{k_{1} k_{1 z}} \sum_{b= \pm} \frac{b}{K_{z}-b k_{1 z}} \sum_{n} \chi_{n} \sqrt{n(n+1)} \\
& \times\left[N_{n}\left(\widehat{\mathbf{s}} \cdot \widehat{\mathbf{s}}_{b}\right) T_{n}^{1} x_{b \varphi n}^{1}-M_{n}\left(\widehat{\mathbf{s}} \cdot \widehat{\mathbf{s}}_{b}\right) T_{n}^{2} x_{b \varphi n}^{2}\right]
\end{aligned}
$$

for a $\varphi$-polarized incidence.

Eq. (176). Inserting Eqs. (146)-(147) together with Eqs. (133)-

(134) in Eq. (176), we find that for a $\eta$-polarized incidence,

$$
0=\sum_{b= \pm} \frac{b}{K_{\mathrm{z}}+b k_{1 \mathrm{z}}} \mathrm{e}^{\mathrm{j} b K_{\mathrm{z}} H}
$$

$$
\begin{aligned}
& \times \sum_{m^{\prime} n^{\prime}}\left\{\mathbf{m}_{-m n}\left(\widehat{\mathbf{S}}_{\mathrm{R}}\right) \cdot\left\{\left[\mathbf{m}_{m^{\prime} n^{\prime}}\left(\widehat{\mathbf{s}}_{\mathrm{R}}\right) \otimes \mathbf{m}_{-m^{\prime} n^{\prime}}\left(\widehat{\mathbf{s}}_{b}\right)\right] \cdot \widehat{\boldsymbol{\eta}}\left(\widehat{\mathbf{s}}_{b}\right)\right\} T_{n^{\prime}}^{1} x_{b \eta n^{\prime}}^{1}\right. \\
& \left.-\mathbf{n}_{-m n}\left(\widehat{\mathbf{S}}_{\mathrm{R}}\right) \cdot\left\{\left[\mathbf{m}_{m^{\prime} n^{\prime}}\left(\widehat{\mathbf{S}}_{\mathrm{R}}\right) \otimes \mathbf{n}_{-m^{\prime} n^{\prime}}\left(\widehat{\mathbf{s}}_{b}\right)\right] \cdot \widehat{\boldsymbol{\eta}}\left(\widehat{\mathbf{s}}_{b}\right)\right\} T_{n^{\prime}}^{2} x_{b \eta n^{\prime}}^{2}\right\}
\end{aligned}
$$

and

$$
\begin{aligned}
0 & =\sum_{b= \pm} \frac{b}{K_{z}+b k_{1 z}} \mathrm{e}^{\mathrm{j} b K_{z} H} \\
& \times \sum_{m^{\prime} n^{\prime}}\left\{\mathbf{n}_{-m n}\left(\widehat{\mathbf{s}}_{\mathrm{R}}\right) \cdot\left\{\left[\mathbf{m}_{m^{\prime} n^{\prime}}\left(\widehat{\mathbf{s}}_{\mathrm{R}}\right) \otimes \mathbf{m}_{-m^{\prime} n^{\prime}}\left(\widehat{\mathbf{s}}_{b}\right)\right] \cdot \widehat{\boldsymbol{\eta}}\left(\widehat{\mathbf{s}}_{b}\right)\right\} T_{n^{\prime}}^{1} 1_{b \eta n^{\prime}}^{1}\right. \\
& \left.+\mathbf{m}_{-m n}\left(\widehat{\mathbf{s}}_{\mathrm{R}}\right) \cdot\left\{\left[\mathbf{m}_{m^{\prime} n^{\prime}}\left(\widehat{\mathbf{S}}_{\mathrm{R}}\right) \otimes \mathbf{n}_{-m^{\prime} n^{\prime}}\left(\widehat{\mathbf{s}}_{b}\right)\right] \cdot \widehat{\boldsymbol{\eta}}\left(\widehat{\mathbf{s}}_{b}\right)\right\} T_{n^{\prime}}^{2} x_{b \eta n^{\prime}}^{2}\right\}
\end{aligned}
$$

Using again Eqs. (177), (178), (180), and (181), we get

$$
\begin{aligned}
0 & =\sum_{b= \pm} \frac{b}{K_{\mathrm{z}}+b k_{1 \mathrm{z}}} \mathrm{e}^{\mathrm{j} b K_{\mathrm{z}} H} \sum_{n} \chi_{n} \sqrt{n(n+1)} \\
& \times\left[M_{n}\left(\widehat{\mathbf{s}}_{\mathrm{R}} \cdot \widehat{\mathbf{s}}_{b}\right) T_{n}^{1} x_{b \theta n}^{1}-N_{n}\left(\widehat{\mathbf{s}}_{\mathrm{R}} \cdot \widehat{\mathbf{s}}_{b}\right) T_{n}^{2} \chi_{b \theta n}^{2}\right]
\end{aligned}
$$

for a $\theta$-polarized incidence, and

$$
\begin{aligned}
0 & =\sum_{b= \pm} \frac{b}{K_{\mathrm{z}}+b k_{1 \mathrm{z}}} \mathrm{e}^{\mathrm{j} b K_{\mathrm{z}} H} \sum_{n^{\prime}} \chi_{n} \sqrt{n(n+1)} \\
& \times\left[N_{n}\left(\widehat{\mathbf{s}}_{\mathrm{R}} \cdot \widehat{\mathbf{s}}_{b}\right) T_{n}^{1} x_{b \varphi n}^{1}-M_{n}\left(\widehat{\mathbf{s}}_{\mathrm{R}} \cdot \widehat{\mathbf{s}}_{b}\right) T_{n}^{2} x_{b \varphi n}^{2}\right]
\end{aligned}
$$

for a $\varphi$-polarized incidence.

Thus, the two systems of equations of the generalized EwaldOseen extinction theorem reduce to two scalar equations that are polarization dependent. These are Eqs. (188) and (192) for a $\theta$ polarized incidence, and Eqs. (189) and (193) for a $\varphi$-polarized incidence.

In summary, the procedure for computing the configurationaveraged exciting field involves the following steps:

1. solve the dispersion equation (163) for the effective wavenumber $K$;

2. solve the homogeneous system of equations (159)-(160) together with the two scalar equations (188) and (192), or (189) and (193), for the coefficients $x_{b \eta n}^{1,2}$;

3. compute the vectors $e_{b \eta}$ from Eqs. (145)-(147);

4. for a $\eta$-polarized incidence, compute the configurationaveraged field exciting particle $i$ at the field point $\mathbf{r}=\mathbf{r}_{i}+\mathbf{R}_{i}$ according to

$$
\begin{aligned}
& \left\langle\mathbf{E}_{\text {exci }}(\mathbf{r})\right\rangle_{i}=\left\langle\mathbf{E}_{\mathrm{exci}}^{(i)}\left(\mathbf{r}_{i}\right)\right\rangle_{i}=\mathbf{X}_{1}^{T}\left(k_{1} \mathbf{r}_{i}\right)\left\langle\mathrm{e}_{i \eta}\right\rangle_{i}, \\
& \text { where }\left\langle\mathrm{e}_{i \eta}\right\rangle_{i}=\sum_{b= \pm} \exp \left(\mathbf{j} \mathbf{K}_{b} \cdot \mathbf{R}_{i}\right) \mathrm{e}_{b \eta} .
\end{aligned}
$$

The following two results, established by a numerical analysis, will play an important role in further development. Referring to Figs. 8 and 9, these are

1. $\left|x_{-\eta \eta}^{1,2}\right| \ll\left|x_{+\eta \eta}^{1,2}\right|$, and

2. $x_{+n}^{1,2} \approx 1$ for small values of the volume concentration $f$, e.g., $f=0.01$.

Because the coefficients $x_{+\eta n n}^{1,2}$ and $x_{-\eta n}^{1,2}$ correspond to an upgoing and a down-going wave, respectively, the first result which is an agreement with that of Ref. [32] suggests that the coherent field reflected by a layer with non-scattering boundaries is much weaker than the coherent transmitted field. On the other had, in view of Eqs. (143)-(144) and (146)-(147), the second result suggests that for sparse media, the conditional configuration-averaged exciting field can be considered to be approximately equal to the incident field (provided that $K \approx k_{1}$ and $\widehat{\mathbf{s}}_{\mathrm{T}} \approx \widehat{\mathbf{s}}$ ). 

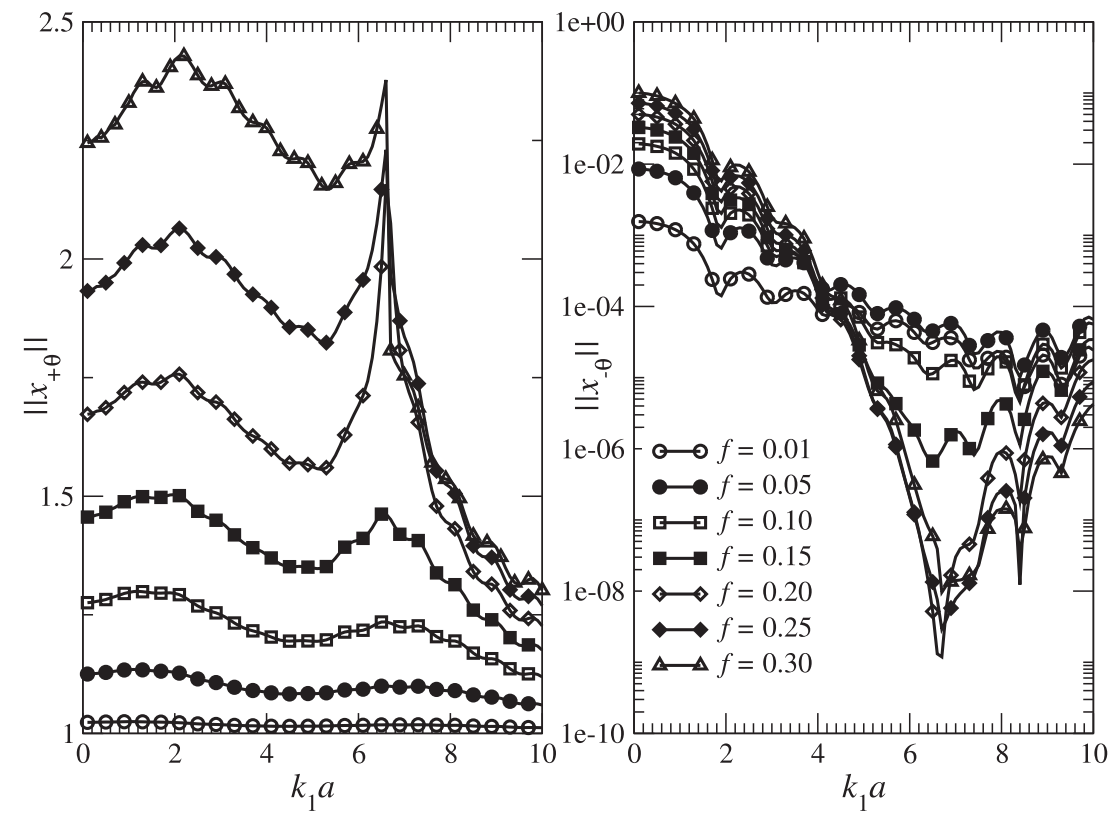

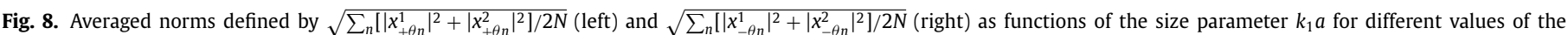

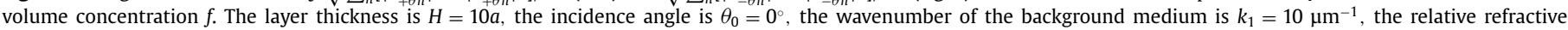
index of the particles is $m=1.33$, and the maximum expansion order is $N_{\text {rank }}=15$.

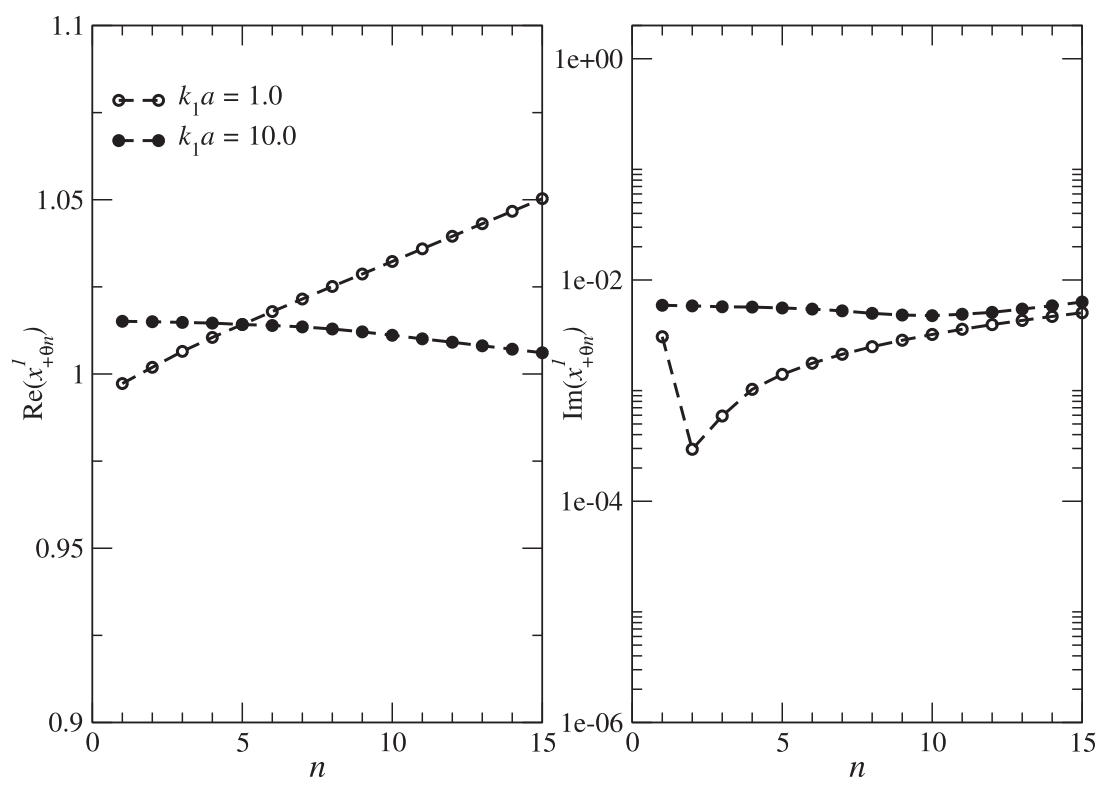

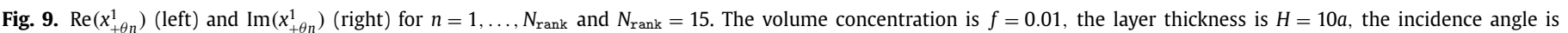

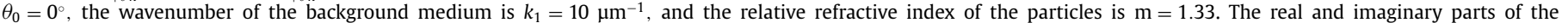
coefficients $x_{+\theta n}^{2}$ have the same dependency on $n$.

\section{Discussion}

In this paper we have considered the scattering of a plane electromagnetic wave obliquely incident on a discrete random layer with non-scattering boundaries. We solved the Lax integral equation for the conditional configuration-averaged exciting field coefficients by applying the technique used by Tsang and Kong $[5,23]$ for a semi-infinite discrete random medium. Some particulars of the solution method are summarized below:

1. The conditional configuration-averaged exciting field coefficients are expressed as a linear combination of coefficients corresponding to an up-going and a down-going wave, that is, $\left\langle\mathrm{e}_{i}\right\rangle_{i}\left(\mathbf{R}_{i}\right)=\sum_{b= \pm} \exp \left(\mathrm{j} \mathbf{K}_{b} \cdot \mathbf{R}_{i}\right) \mathrm{e}_{b}$. This special-form solution representation is assumed to be valid in the whole domain occupied by the particles. In other words, the analysis is restricted to the computation of the zeroth-order fields without a special treatment of the critical domains.

2. By balancing the waves with different propagation directions and wavenumbers in the Lax integral equation, we obtained two homogeneous systems of equations corresponding to the generalized Lorenz-Lorentz law and two inhomogeneous systems of equations corresponding to the generalized Ewald-Oseen extinction theorem.

3. It is shown that even in the case of a discrete random layer, (i) the two homogeneous systems of equations of the generalized Lorenz-Lorentz law reduce to a single homogeneous 
system of equations corresponding to a semi-infinite discrete random medium at normal incidence, and (ii) the dispersion equation is direction and polarization independent. It is also shown that the two inhomogeneous systems of equations of the generalized Ewald-Oseen extinction theorem can be reduced to two scalar equations by means of the addition theorem for vector spherical harmonics.

We conclude our analysis by presenting some new results related to the representation of the conditional configurationaveraged exciting field coefficients. The goal of this presentation is to examine if and how these results can be applied to the scattering problem considered in this study.

\subsection{Result 1}

An approach consisting in the solution of the integral equation (138), without assuming a special form for the conditional configuration-averaged exciting field coefficients, has been proposed by Kristensson [33]. To reduce the complexity of the problem, the case of normal incidence was considered. Here, we discuss a possible extension of this approach to the general case of oblique incidence. For a $\eta$-polarized incident field, we assume that the solution is of the form

$\left\langle\mathrm{e}_{i \eta}\right\rangle_{i}\left(\mathbf{R}_{i}\right)=\mathrm{e}^{\mathrm{j}^{\mathbf{k} \mathbf{k}_{1 \leq \perp} \cdot \mathbf{R}_{i \perp}}} \overline{\mathrm{e}}_{\eta}\left(z_{i}\right), \quad 0 \leq z_{i} \leq H$.

Thus, the solution representation (194) is assumed to be valid in the entire domain of analysis. Substituting Eq. (194) into Eq. (138), we obtain

$$
\begin{aligned}
\overline{\mathrm{e}}_{\eta}\left(z_{i}\right) & =\mathrm{e}^{\mathrm{j} k_{1 z} z_{i}} \mathrm{e}_{0 \eta}+n_{0} \int_{D-D_{2 a}\left(\mathbf{R}_{i}\right)} \mathrm{Q}\left(-k_{1} \mathbf{R}_{j i}\right) \\
& \times \mathrm{e}^{\mathrm{j} \mathbf{k}_{1 \mathrm{~s} \perp} \cdot \mathbf{R}_{j i \perp}} \overline{\mathrm{e}}_{\eta}\left(z_{j}\right) g\left(R_{j i}\right) \mathrm{d}^{3} \mathbf{R}_{j}
\end{aligned}
$$

with $k_{1 \mathrm{z}}=k_{1 \mathrm{z}}\left(\mathbf{k}_{1 \mathrm{~s} \perp}\right)=\sqrt{k_{1}^{2}-k_{1 \mathrm{~s} \perp}^{2}}$ and $k_{1 \mathrm{~s} \perp}=k_{1} \sin \theta_{0}$. Taking into account the representations of the elements of the translation matrix as given by Eqs. (80)-(82), we see that the following integral is of interest in computing the right-hand side of Eq. (195):

$$
\begin{aligned}
I\left(z_{i} ; \overline{\mathrm{e}}_{\eta}\right) & =\int_{D-D_{2 a}\left(\mathbf{R}_{i}\right)} u_{m^{\prime}-m, n^{\prime \prime}}^{3}\left(k_{1} \mathbf{R}_{j i}\right) \mathrm{e}^{\mathrm{j} \mathbf{k}_{1 s \perp} \cdot \mathbf{R}_{j \perp \perp}} \\
& \times \overline{\mathrm{e}}_{\eta}\left(z_{j}\right) g\left(R_{j i}\right) \mathrm{d}^{2} \mathbf{R}_{j i \perp} \mathrm{d} z_{j} .
\end{aligned}
$$

To simplify the analysis, we consider the hole-correction approximation for the pair correlation function, i.e., $g\left(R_{j i}\right)=0$ for $0 \leq R_{j i}<2 a$, and $g\left(R_{i j}\right)=1$ for $R_{j i} \geq 2 a$. The interval of integration over $z_{j},[0, H]$, is split into several (sub)intervals; in these intervals, the plane $z=z_{j}$ does or does not intersect the (complete or truncated) sphere $D_{2 a}\left(\mathbf{R}_{i}\right)$. We distinguish the following situations.

1. For $z_{i}<H-2 a$, the plane $z=z_{j}$ does not intersect the sphere $D_{2 a}\left(\mathbf{R}_{i}\right)$ when $z_{j}$ ranges in the interval $\left[z_{i}+2 a, H\right]$. Using the integral representations of the radiating spherical waves in terms of plane waves in the case $z_{i}<z_{j}$ (compare with Eq. (94)),

$u_{m n}^{3}\left(k \mathbf{R}_{j i}\right)=\frac{1}{2 \pi \mathrm{j}^{n}} \int_{-\infty}^{\infty} Y_{m n}\left(\widehat{\mathbf{k}}^{+}\right) \mathrm{e}^{\mathrm{j} \mathbf{k}_{\perp} \cdot \mathbf{R}_{j \perp \perp}} \mathrm{e}^{\mathrm{j} k_{1 z}\left(\mathbf{k}_{\perp}\right)\left(z_{j}-z_{i}\right)} \frac{\mathrm{d}^{2} \mathbf{k}_{\perp}}{k k_{1 \mathrm{z}}\left(\mathbf{k}_{\perp}\right)}$

with $\mathbf{k}^{+}=\mathbf{k}_{\perp}+k_{1 \mathrm{z}}\left(\mathbf{k}_{\perp}\right) \widehat{\mathbf{z}}$, we find

$$
\begin{aligned}
I\left(z_{i} ; \overline{\mathrm{e}}_{\eta}\right) & =\int_{z_{i}+2 a}^{H}\left[\int_{\mathbb{R}^{2}} u_{m^{\prime}-m, n^{\prime \prime}}^{3}\left(k_{1} \mathbf{R}_{j i}\right) \mathrm{e}^{\mathrm{j} \mathbf{k}_{1 \mathrm{~s} \perp} \cdot \mathbf{R}_{j \perp \perp}}\right. \\
& \left.\times \overline{\mathrm{e}}_{\eta}\left(z_{j}\right) \mathrm{d}^{2} \mathbf{R}_{j i \perp}\right] \mathrm{d} z_{j}
\end{aligned}
$$

$$
=\frac{2 \pi \mathrm{j}^{n^{\prime \prime}}}{k_{1} k_{1 z}} Y_{m^{\prime}-m, n^{\prime \prime}}\left(\widehat{\mathbf{k}}_{1 \mathrm{R}}\right) \int_{z_{i}+2 a}^{H} \mathrm{e}^{\mathrm{j} k_{1 z}\left(z_{j}-z_{i}\right)} \overline{\mathrm{e}}_{\eta}\left(z_{j}\right) \mathrm{d} z_{j},
$$

where (cf. Eq. (130)) $\mathbf{k}_{1 \mathrm{R}}=\mathbf{k}_{1 \mathrm{~s} \perp}-k_{1 \mathrm{z}}$.

2. For $z_{i}>2 a$, the plane $z=z_{j}$ does not intersect the sphere $D_{2 a}\left(\mathbf{R}_{i}\right)$ when $z_{j}$ ranges in the interval $\left[0, z_{i}-2 a\right]$. Using the integral representations of the radiating spherical waves in terms of plane waves in the case $z_{i}>z_{j}$ (compare with Eq. (93)),

$$
\begin{aligned}
u_{m n}^{3}\left(k \mathbf{R}_{j i}\right)= & \frac{1}{2 \pi \mathrm{j}^{n}} \int_{-\infty}^{\infty} Y_{m n}\left(\widehat{\mathbf{k}}^{-}\right) \mathrm{e}^{\mathrm{j} \mathbf{k}_{\perp} \cdot \mathbf{R}_{j i \perp}} \mathrm{e}^{-\mathrm{j} k_{1 z}\left(\mathbf{k}_{\perp}\right)\left(z_{j}-z_{i}\right)} \\
& \times \frac{\mathrm{d}^{2} \mathbf{k}_{\perp}}{k k_{1 z}\left(\mathbf{k}_{\perp}\right)}
\end{aligned}
$$

with $\mathbf{k}^{-}=\mathbf{k}_{\perp}-k_{1 \mathrm{z}}\left(\mathbf{k}_{\perp}\right) \widehat{\mathbf{z}}$, we find

$$
\begin{aligned}
I\left(z_{i} ; \overline{\mathrm{e}}_{\eta}\right) & =\int_{0}^{z_{i}-2 a}\left[\int_{\mathbb{R}^{2}} u_{m^{\prime}-m, n^{\prime \prime}}^{3}\left(k_{1} \mathbf{R}_{j i}\right) \mathrm{e}^{\mathrm{j} \mathbf{k}_{1 \mathrm{~s} \perp} \cdot \mathbf{R}_{j \perp \perp}}\right. \\
& \left.\times \overline{\mathrm{e}}_{\eta}\left(z_{j}\right) \mathrm{d}^{2} \mathbf{R}_{j i \perp}\right] \mathrm{d} z_{j} \\
& =\frac{2 \pi \mathrm{j}^{n^{\prime \prime}}}{k_{1} k_{1 z}} Y_{m^{\prime}-m, n^{\prime \prime}}\left(\widehat{\mathbf{k}}_{1 \mathrm{~s}}\right) \int_{0}^{z_{i}-2 a} \mathrm{e}^{\mathrm{j} k_{1 z}\left(z_{i}-z_{j}\right)} \overline{\mathrm{e}}_{\eta}\left(z_{j}\right) \mathrm{d} z_{j} .
\end{aligned}
$$

3. The plane $z=z_{j}$ crosses the sphere $D_{2 a}\left(\mathbf{R}_{i}\right)$ when $z_{j}$ ranges in the interval of integration. Using the integral representation of the cylindrical Bessel functions

$$
J_{n}(x)=\frac{1}{2 \pi \mathrm{j}^{n}} \int_{0}^{2 \pi} \mathrm{e}^{\mathrm{j} n \varphi} \mathrm{e}^{\mathrm{j} x \cos \varphi} \mathrm{d} \varphi,
$$

we find

$$
\begin{aligned}
I\left(z_{i} ; \overline{\mathrm{e}}_{\eta}\right) & =\int_{\max \left(0, z_{i}-2 a\right)}^{\min \left(z_{i}+2 a, H\right)}\left\{\int _ { \rho _ { 0 } ( z _ { j i } ) } ^ { \infty } \left[\int_{0}^{2 \pi} u_{m^{\prime}-m, n^{\prime \prime}}^{3}\left(k_{1} R_{j i}, \theta_{j i}, \varphi_{j i}\right)\right.\right. \\
& \left.\left.\times \mathrm{e}^{\mathrm{j} k_{1 \mathrm{~s} \perp} \rho_{j i} \cos \left(\varphi_{j i}-\varphi_{0}\right)} \mathrm{d} \varphi_{j i}\right] \rho_{j i} \mathrm{~d} \rho_{j i}\right\} \overline{\mathrm{e}}_{\eta}\left(z_{j}\right) \mathrm{d} z_{j} \\
= & 2 \pi \mathrm{j}^{m^{\prime}-m} \mathrm{e}^{\mathrm{j}\left(m^{\prime}-m\right) \varphi_{0}} \int_{\max \left(0, z_{i}-2 a\right)}^{\min \left(z_{i}+2 a, H\right)} \\
& \times\left[\int_{\rho_{0}\left(z_{j i}\right)}^{\infty} h_{n^{\prime \prime}}\left(k_{1} \sqrt{\rho_{j i}^{2}+z_{j i}^{2}}\right) P_{n^{\prime \prime}}^{\left|m^{\prime}-m\right|}\left(\frac{z_{j i}}{\sqrt{\rho_{j i}^{2}+z_{j i}^{2}}}\right)\right. \\
& \left.\times J_{m^{\prime}-m}\left(k_{1 \mathrm{~s} \perp} \rho_{j i}\right) \rho_{j i} \mathrm{~d} \rho_{j i}\right] \overline{\mathrm{e}}_{\eta}\left(z_{j}\right) \mathrm{d} z_{j},
\end{aligned}
$$

where $\left(R_{j i}, \theta_{j i}, \varphi_{j i}\right)$ and $\left(z_{j i}, \rho_{j i}, \varphi_{j i}\right)$ are the spherical and the cylindrical coordinates of $\mathbf{R}_{j i}$, respectively, $z_{j i}=z_{j}-z_{i}, R_{j i}=$

$$
\begin{aligned}
& \sqrt{\rho_{j i}^{2}+z_{j i}^{2}}, \cos \theta_{j i}=z_{j i} / \sqrt{\rho_{j i}^{2}+z_{j i}^{2}}, \text { and } \\
& \rho_{0}\left(z_{j i}\right)=\sqrt{4 a^{2}-\left(z_{j}-z_{i}\right)^{2}} .
\end{aligned}
$$

In the last case, the main problem which has to be solved is the computation of the integral over $\rho_{j i}$. This computational aspect is discussed in Appendix C. The integral equation can then be discretized at equally spaced points $z_{i}$ in the interval $[0, H]$, and the integrals over $z_{j}$ in Eqs. (198), (200), and (202) can be computed, for example, by the use of Simpson's quadrature and by assuming that $\overline{\mathrm{e}}_{\eta}(z)$ varies linearly between the points of discretization $z_{i}[32]$.

\subsection{Result 2}

Gower et al. [35] suggested that in general there is not only one effective wavenumber $K=K_{1}$, but there is a series of effective wavnumbers $K_{1}, K_{2}, \ldots, K_{N_{K}}$ in a discrete random medium. The 
majority of these waves are highly attenuating, i.e., $K_{p}$ often has a large imaginary part for $p>1$, so that the least attenuating, say $K_{1}$, will dominate the coherent field inside the particulate medium. However, through a numerical analysis, it is has been shown that these waves can make a significant contribution to the reflection and transmission of the layer beyond the low-frequency regime. The analysis in Ref. [35] is performed for the scalar wave equation in two spatial dimensions, and considers particles placed in a semi-infinite medium. In the following, we extend this idea to the case of electromagnetic scattering by a discrete random layer. For the effective wavenumbers $K_{p}$ with $p=1, \ldots, N_{K}$, we assume the solution representation

$\left\langle\mathrm{e}_{i \eta}\right\rangle_{i}\left(\mathbf{R}_{i}\right)=\sum_{p=1}^{N_{K}} \sum_{b= \pm} \mathrm{e}^{\mathrm{j} \mathbf{K}_{p b} \cdot \mathbf{R}_{i}} \mathrm{e}_{p b \eta}$,

where

$\mathbf{K}_{p b}=\mathbf{K}_{\perp}+b K_{p z}\left(\mathbf{K}_{\perp}\right) \hat{\mathbf{z}}$,

$K_{p z}\left(\mathbf{K}_{\perp}\right)=\sqrt{K_{p}^{2}-K_{\perp}^{2}}$.

In addition, we suppose that the representation (203) is valid for all $z_{i}$ in $D$ even in the critical domains $0 \leq z_{i}<2 a$ and $H-2 a<z_{i} \leq$ $H$. Repeating the steps of our previous derivation, we find that the generalized Lorenz-Lorentz law and the generalized Ewald-Oseen extinction theorem become

$\mathrm{e}_{p b \eta}=n_{0}\left[\mathrm{~J}_{1 a}^{b}\left(K_{p}\right)+\mathrm{J}_{2 a}^{b}\left(K_{p}\right)\right] \mathrm{Te}_{p b \eta}$,

and

$\mathrm{e}_{0 \eta}+n_{0} \sum_{p=1}^{N_{K}} \sum_{b= \pm} \mathrm{J}_{2 \mathrm{z} 0}^{b}\left(K_{p}\right) \mathrm{Te}_{p b \eta}=0$,

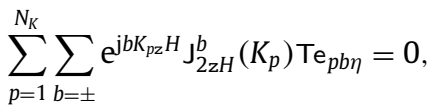

respectively. Here, the dependency of the matrices $\mathrm{J}_{1 a}^{b}, \mathrm{~J}_{2 a}^{b}, \mathrm{~J}_{2 \mathrm{z} 0}^{b}$, and $J_{2 z H}^{b}$ on the effective wavenumber $K_{p}$ is indicated explicitly. Thus, the homogenous system of equations of the generalized Lorenz-Lorentz law remains unchanged, and the effective wavenumbers $K_{1}, K_{2}, \ldots, K_{N_{K}}$ are the solutions of the dispersion equation (163). To reduce the systems of equations of the generalized Ewald-Oseen extinction theorem to two polarizationdependent scalar equations, we set $\mathrm{e}_{p b \eta}=\left[e_{p b \eta m n}^{1}, e_{p b \eta m n}^{2}\right]^{T}$,

$e_{p b \eta m n}^{1}=4 \pi \mathrm{j}^{n} \widehat{\boldsymbol{\eta}}\left(\widehat{\mathbf{s}}_{p b}\right) \cdot \mathbf{m}_{-m n}\left(\widehat{\mathbf{s}}_{p b}\right) x_{p b \eta n}^{1}$,

$e_{p b \eta m n}^{2}=-4 \pi \mathrm{j}^{n+1} \widehat{\boldsymbol{\eta}}\left(\widehat{\mathbf{s}}_{p b}\right) \cdot \mathbf{n}_{-m n}\left(\widehat{\mathbf{s}}_{p b}\right) x_{p b \eta n}^{2}$,

where $K_{p} \widehat{\mathbf{s}}_{p b}=\mathbf{k}_{1 \mathrm{~s} \perp}+b K_{p \mathrm{z}} \hat{\mathbf{z}}$ and $K_{p \mathrm{z}}=\sqrt{K_{p}^{2}-k_{1 \mathrm{~s} \perp}^{2}}$. Then, we obtain

$$
\begin{aligned}
1 & =-\mathrm{j} \frac{8 \pi^{2} n_{0}}{k_{1} k_{1 \mathrm{z}}} \sum_{p=1}^{N_{K}} \sum_{b= \pm} \frac{b}{K_{p z}-b k_{1 \mathrm{z}}} \sum_{n} \chi_{n} \sqrt{n(n+1)} \\
& \times\left[M_{n}\left(\widehat{\mathbf{s}} \cdot \widehat{\mathbf{s}}_{p b}\right) T_{n}^{1} x_{p b \theta n}^{1}-N_{n}\left(\widehat{\mathbf{s}} \cdot \widehat{\mathbf{s}}_{p b}\right) T_{n}^{2} x_{p b \theta n}^{2}\right]
\end{aligned}
$$

$$
\begin{aligned}
0 & =\sum_{p=1}^{N_{K}} \sum_{b= \pm} \frac{b}{K_{p \mathrm{z}}+b k_{1 \mathrm{z}}} \mathrm{e}^{\mathrm{j} b K_{p \mathrm{z}} H} \sum_{n} \chi_{n} \sqrt{n(n+1)} \\
& \times\left[M_{n}\left(\widehat{\mathbf{s}}_{\mathrm{R}} \cdot \widehat{\mathbf{s}}_{p b}\right) T_{n}^{1} x_{p b \theta n}^{1}-N_{n}\left(\widehat{\mathbf{s}}_{\mathrm{R}} \cdot \widehat{\mathbf{s}}_{p b}\right) T_{n}^{2} \chi_{p b \theta n}^{2}\right]
\end{aligned}
$$

for a $\theta$-polarized incidence, and

$$
\begin{aligned}
1 & =\mathrm{j} \frac{8 \pi^{2} n_{0}}{k_{1} k_{1 \mathrm{z}}} \sum_{p=1}^{N_{K}} \sum_{b= \pm} \frac{b}{K_{\mathrm{pz}}-b k_{1 \mathrm{z}}} \sum_{n} \chi_{n} \sqrt{n(n+1)} \\
& \times\left[N_{n}\left(\widehat{\mathbf{s}} \cdot \widehat{\mathbf{s}}_{p b}\right) T_{n}^{1} x_{p b \varphi n}^{1}-M_{n}\left(\widehat{\mathbf{s}} \cdot \widehat{\mathbf{s}}_{p b}\right) T_{n}^{2} x_{p b \varphi n}^{2}\right], \\
0 & =\sum_{p=1}^{N_{K}} \sum_{b= \pm} \frac{b}{K_{p z}+b k_{1 \mathrm{z}}} \mathrm{e}^{\mathrm{j} b K_{p z} H} \sum_{n^{\prime}} \chi_{n} \sqrt{n(n+1)} \\
& \times\left[N_{n}\left(\widehat{\mathbf{s}}_{\mathrm{R}} \cdot \widehat{\mathbf{s}}_{p b}\right) T_{n}^{1} x_{p b \varphi n}^{1}-M_{n}\left(\widehat{\mathbf{s}}_{\mathrm{R}} \cdot \widehat{\mathbf{s}}_{p b}\right) T_{n}^{2} x_{p b \varphi n}^{2}\right]
\end{aligned}
$$

for a $\varphi$-polarized incidence. The scalar equations (211) and (212), as well as the scalar equations (213) and (214), have $2 N_{K}$ unknowns ( 2 scalars for each wavenumber $K_{p}$ with $p=1, \ldots, N_{K}$ ). Thus, the only option to determine $e_{p b \eta}$ (under the assumption that the representation (203) is valid for all $z_{i}$ in $D$ ) is to solve these equations in the least squares sense.

\subsection{Result 3}

For the acoustic scattering by parallel identical circular cylinders randomly distributed in a semi-infinite medium, Linton and Martin [36] considered a more general solution representation than in Eq. (139). In the case of electromagnetic scattering by a discrete random layer, this representation is

$\left\langle\mathrm{e}_{i \eta}\right\rangle_{i}\left(\mathbf{R}_{i}\right)=\left\{\begin{array}{l}\mathrm{e}^{\mathrm{j} \mathbf{K}_{\perp} \cdot \mathbf{R}_{i \perp}} \overline{\mathrm{e}}_{+\eta}\left(z_{i}\right), 0 \leq z_{i} \leq h, \\ \sum_{b= \pm} \mathrm{e}^{\mathrm{j} \mathbf{K}_{b} \cdot \mathbf{R}_{i}} e_{b \eta}, h \leq z_{i} \leq H-h, \\ \mathrm{e}^{\mathrm{j} \mathbf{K}_{\perp} \cdot \mathbf{R}_{i \perp} \overline{\mathrm{e}}_{-\eta}}\left(z_{i}\right), H-h \leq z_{i} \leq H,\end{array}\right.$

with a sufficiently large $h$. Thus, we do not assume that the conditional configuration-averaged exciting field coefficients are proportional to $\exp \left(\mathrm{j} \mathbf{K}_{b} \cdot \mathbf{R}_{i}\right)$ everywhere inside the domain $D$, but only in the domain $h \leq z_{i} \leq H-h$ away from the boundary. Defining the plane-parallel domains

$D_{h}^{+}=\left\{(x, y, z) \mid x, y \in \mathbb{R}^{2}, \quad 0 \leq z \leq h\right\}$,

$D_{h}=\left\{(x, y, z) \mid x, y \in \mathbb{R}^{2}, \quad h \leq z \leq H-h\right\}$,

$D_{h}^{-}=\left\{(x, y, z) \mid x, y \in \mathbb{R}^{2}, \quad H-h \leq z \leq H\right\}$,

and substituting Eq. (215) in Eq. (138) gives for $h+2 a \leq z_{i} \leq H-$ $h-2 a$,

$$
\begin{aligned}
\sum_{b= \pm} \mathrm{e}^{\mathrm{j}^{\mathbf{K}} \cdot \mathbf{R}_{i}} \mathrm{e}_{b \eta} & =\mathrm{e}^{\mathrm{j} \mathbf{k}_{1 \mathrm{~s}} \cdot \mathbf{R}_{i}} \mathrm{e}_{0 \eta}+n_{0} \sum_{b= \pm} \int_{D_{h}-D_{2 a}\left(\mathbf{R}_{i}\right)} \mathrm{Q}\left(-k_{1} \mathbf{R}_{j i}\right) \\
& \times \mathrm{e}^{\mathrm{j} \mathbf{K}_{b} \cdot \mathbf{R}_{j}} \mathrm{e}_{b \eta} g\left(R_{j i}\right) \mathrm{d}^{3} \mathbf{R}_{j} \\
& +n_{0} \sum_{b= \pm} \int_{D_{h}^{b}} \mathrm{Q}\left(-k_{1} \mathbf{R}_{j i}\right) \mathrm{e}^{\mathrm{j} \mathbf{K}_{\perp} \cdot \mathbf{R}_{j \perp}} \overline{\mathrm{e}}_{b \eta}\left(z_{j}\right) \mathrm{d}^{3} \mathbf{R}_{j} .
\end{aligned}
$$

Here, it is assumed that for $\mathbf{R}_{j} \in D_{h}^{b}$ and $\mathbf{R}_{i} \in D_{h}$, we can approximate $g\left(R_{i j}\right) \approx 1$ (eventually, we can restrict $z_{i}$ to the domain $h+2 a \leq H_{\min } \leq z_{i} \leq H_{\max } \leq H-h-2 a$, and choose $H_{\min }$ and $H_{\max }$ such that this approximation is valid). Consider the last integral in Eq. (219) which we denote by $\mathrm{j}^{b}\left(\mathbf{R}_{i} ; \overline{\mathrm{e}}_{b \eta}\right)$, that is,

$\mathrm{j}^{b}\left(\mathbf{R}_{i} ; \overline{\mathrm{e}}_{b \eta}\right)=\int_{D_{h}^{b}} \mathcal{T}_{31}^{T}\left(-k_{1} \mathbf{R}_{j i}\right) \mathrm{e}^{\mathrm{j} \mathbf{K}_{\perp} \cdot \mathbf{R}_{j \perp}} \mathrm{T} \overline{\mathrm{e}}_{b \eta}\left(z_{j}\right) \mathrm{d}^{3} \mathbf{R}_{j}$.

Taking into account the representations of the elements of the translation matrix as given by Eqs. (80)-(82), and setting 

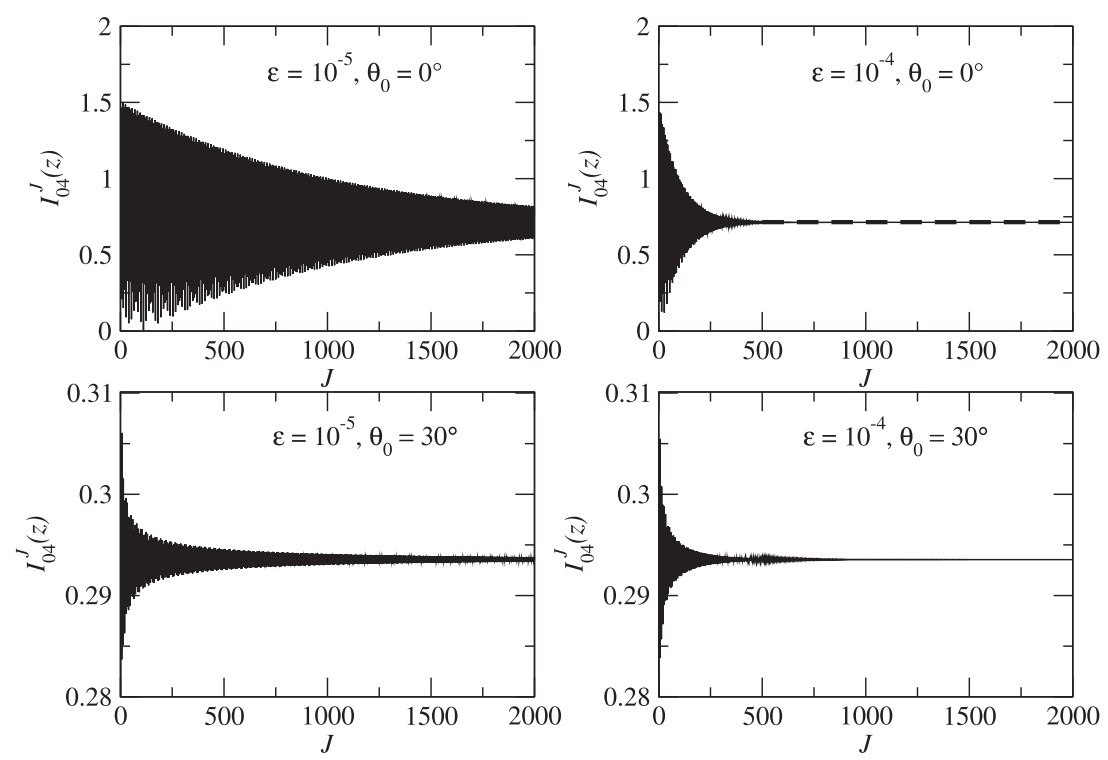

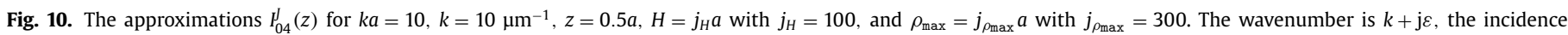
angle is $\theta_{0}$, and the dotted line in the upper right plot corresponds to the value of $I_{04}(z)$ computed by means of Eq. (283).

$\overline{\mathrm{e}}_{b \eta}\left(z_{i}\right)=\left[\bar{e}_{b \eta m n}^{1}\left(z_{i}\right), \bar{e}_{b \eta m n}^{2}\left(z_{i}\right)\right]^{T}$, we see that the computation of the vector $\mathrm{j}^{b}\left(\mathbf{R}_{i} ; \overline{\mathrm{e}}_{b \eta}\right)$ requires the computation of the integral

$$
\begin{aligned}
I^{b}\left(\mathbf{R}_{i} ; \overline{\mathrm{e}}_{b \eta}\right) & =\mathrm{e}^{\mathrm{j} \mathbf{K}_{\perp} \cdot \mathbf{R}_{i \perp}} \int_{D_{h}^{b}} u_{m^{\prime}-m, n^{\prime \prime}}^{3}\left(k_{1} \mathbf{R}_{j i}\right) \mathrm{e}^{j \mathbf{K}_{\perp} \cdot \mathbf{R}_{j i \perp}} \\
& \times \bar{e}_{b \eta m^{\prime} n^{\prime}}^{\alpha}\left(z_{j}\right) \mathrm{d}^{3} \mathbf{R}_{j i}
\end{aligned}
$$

with $\alpha=1,2$. For the integral over $D_{h}^{+}$(the case $z_{i}>z_{j}$ ) we use the integral representations of the radiating spherical waves in terms of plane waves as given by Eq. (199), while for the integral over $D_{h}^{-}$(the case $z_{i}<z_{j}$ ), we use the integral representation (197). We obtain

$$
\begin{aligned}
I^{b}\left(\mathbf{R}_{i} ; \overline{\mathrm{e}}_{b \eta}\right) & =\frac{2 \pi}{k k_{1 \mathrm{z}}\left(\mathbf{K}_{\perp}\right)} \mathrm{j}^{n^{\prime \prime}} \mathrm{e}^{\mathrm{j} \mathbf{K}_{\perp} \cdot \mathbf{R}_{i \perp}} \mathrm{e}^{\mathrm{j} b k_{1 z}\left(\mathbf{K}_{\perp}\right) z_{i}} \\
& \times Y_{m^{\prime}-m, n^{\prime \prime}}\left(\widehat{\mathbf{k}}^{b}\right) K_{m^{\prime} n^{\prime}}^{b \alpha}\left(\mathbf{K}_{\perp} ; \overline{\mathrm{e}}_{b \eta}\right),
\end{aligned}
$$

where

$K_{m^{\prime} n^{\prime}}^{+\alpha}\left(\mathbf{K}_{\perp} ; \overline{\mathrm{e}}_{+\eta}\right)=\int_{0}^{h} \mathrm{e}^{-\mathrm{j} k_{1 z}\left(\mathbf{K}_{\perp}\right) z_{j}} \bar{e}_{+\eta m^{\prime} n^{\prime}}^{\alpha}\left(z_{j}\right) \mathrm{d} z_{j}$,

$K_{m^{\prime} n^{\prime}}^{-\alpha}\left(\mathbf{K}_{\perp} ; \overline{\mathrm{e}}_{-\eta}\right)=\int_{H-h}^{H} \mathrm{e}^{\mathrm{j} k_{1 z}\left(\mathbf{K}_{\perp}\right) z_{j}} \bar{e}_{-\eta m^{\prime} n^{\prime}}^{\alpha}\left(z_{j}\right) \mathrm{d} z_{j}$,

and $\mathbf{k}^{+}$and $\mathbf{k}^{-}$are given by Eqs. (104) and (108), respectively. This partial result yields

$\mathrm{j}^{b}\left(\mathbf{R}_{i} ; \overline{\mathrm{e}}_{b \eta}\right)=\mathrm{e}^{\mathrm{j} \mathbf{K}_{\perp} \cdot \mathbf{R}_{i \perp}} \mathrm{e}^{\mathrm{j} b k_{1 \mathrm{z}}\left(\mathbf{K}_{\perp}\right) z_{i}} \mathrm{j}_{\mathrm{z}}^{b}\left(\mathbf{R}_{i} ; \overline{\mathrm{e}}_{b \eta}\right)$,

where for

$j_{z}^{b}\left(\mathbf{R}_{i} ; \overline{\mathrm{e}}_{b \eta}\right)=\left[\begin{array}{l}\left(j_{\mathrm{z}}^{b}\left(\mathbf{R}_{i} ; \overline{\mathrm{e}}_{b \eta}\right)\right)_{m n}^{1} \\ \left(j_{\mathrm{z}}^{b}\left(\mathbf{R}_{i} ; \overline{\mathrm{e}}_{b \eta}\right)\right)_{m n}^{2}\end{array}\right]$,

we have

$$
\begin{aligned}
\left(j_{\mathrm{z}}^{b}\left(\mathbf{R}_{i} ; \overline{\mathrm{e}}_{b \eta}\right)\right)_{m n}^{1} & =\frac{2 \pi}{k k_{1 \mathrm{z}}\left(\mathbf{K}_{\perp}\right)} \sum_{m^{\prime} n^{\prime} n^{\prime \prime}} c_{n n^{\prime}}(-1)^{n^{\prime \prime}} Y_{m^{\prime}-m, n^{\prime \prime}}\left(\widehat{\mathbf{k}}^{b}\right) \\
& \times\left[a_{1}\left(-m, n\left|-m^{\prime}, n^{\prime}\right| n^{\prime \prime}\right) T_{n^{\prime}}^{1} K_{m^{\prime} n^{\prime}}^{b 1}\left(\mathbf{K}_{\perp} ; \overline{\mathrm{e}}_{b \eta}\right)\right. \\
& \left.+b_{1}\left(-m, n\left|-m^{\prime}, n^{\prime}\right| n^{\prime \prime}\right) T_{n^{\prime}}^{2} K_{m^{\prime} n^{\prime}}^{b 2}\left(\mathbf{K}_{\perp} ; \overline{\mathrm{e}}_{b \eta}\right)\right]
\end{aligned}
$$

and

$$
\left(j_{\mathrm{z}}^{b}\left(\mathbf{R}_{i} ; \overline{\mathrm{e}}_{b \eta}\right)\right)_{m n}^{2}=\frac{2 \pi}{k k_{1 \mathrm{z}}\left(\mathbf{K}_{\perp}\right)} \sum_{m^{\prime} n^{\prime} n^{\prime \prime}} c_{n n^{\prime}}(-1)^{n^{\prime \prime}} Y_{m^{\prime}-m, n^{\prime \prime}}\left(\widehat{\mathbf{k}}^{b}\right)
$$

$$
\begin{aligned}
& \times\left[b_{1}\left(-m, n\left|-m^{\prime}, n^{\prime}\right| n^{\prime \prime}\right) T_{n^{\prime}}^{1} K_{m^{\prime} n^{\prime}}^{b 1}\left(\mathbf{K}_{\perp} ; \overline{\mathrm{e}}_{b \eta}\right)\right. \\
& \left.+a_{1}\left(-m, n\left|-m^{\prime}, n^{\prime}\right| n^{\prime \prime}\right) T_{n^{\prime}}^{2} K_{m^{\prime} n^{\prime}}^{b 2}\left(\mathbf{K}_{\perp} ; \overline{\mathrm{e}}_{b \eta}\right)\right] .
\end{aligned}
$$

The integral over the domain $D_{h}-D_{2 a}\left(\mathbf{R}_{i}\right)$ is computed as before; the difference is that now, the integral representations (93) and (94) are applied on the planes $z_{j}=h$ and $z_{j}=H-h$, respectively. Then, by separating the upward and downward propagating waves in Eq. (219), and by balancing the waves with the wavenumbers $k_{1}$ and $K$, we find that the equations of the generalized LorenzLorentz law remain unchanged, while the equations of the generalized Ewald-Oseen extinction theorem become (compare with Eqs. (175) and (176))

$$
\begin{gathered}
\mathrm{e}_{0 \eta}+n_{0} \mathrm{e}^{-\mathrm{j} k_{1 \mathrm{z}} h} \sum_{b= \pm} \mathrm{J}_{2 \mathrm{z} 0}^{b} \mathrm{Te}_{b \eta}+n_{0} \mathrm{j}_{\mathrm{z}}^{+}\left(\mathbf{R}_{i} ; \overline{\mathrm{e}}_{+\eta}\right)=0, \\
\mathrm{e}^{\mathrm{j} k_{1 \mathrm{z}}(H-h)} \sum_{b= \pm} \mathrm{e}^{\mathrm{j} b K_{\mathrm{z}} H} \mathrm{~J}_{2 \mathrm{z} H}^{b} T \mathrm{e}_{b \eta}+\mathrm{j}_{\mathrm{z}}^{-}\left(\mathbf{R}_{i} ; \overline{\mathrm{e}}_{-\eta}\right)=0 .
\end{gathered}
$$

Thus, we obtain the dispersion Eq. (163) without knowing the behavior of $\left\langle\mathrm{e}_{i \eta}\right\rangle_{i}\left(\mathbf{R}_{i}\right)$ in the domains $0 \leq z \leq h$ and $H-h \leq z \leq H$, which include the critical domains. In fact, the length $h$ needs not be specified if only the calculation of the effective wavenumber is required.

If we take $h=0$ in Eqs. (229) and (230), then the equations of the generalized Ewald-Oseen extinction theorem are given by Eqs. (175) and (176). This approximation was used by Martin [37]. to compute the coherent field. To solve the problem in the case $h \geq 2 a$, we write the integral equation (138) in the domains $D_{h}^{c}$, $c= \pm$, and obtain

$$
\begin{aligned}
& \overline{\mathrm{e}}_{c \eta}\left(z_{i}\right)=\mathrm{e}^{\mathrm{j} k_{1 \mathrm{z}} z_{i}} \mathrm{e}_{0 \eta}+n_{0} \sum_{b= \pm} \int_{D_{h}-D_{2 a}\left(\mathbf{R}_{i}\right)} \mathrm{Q}\left(-k_{1} \mathbf{R}_{j i}\right) \\
& \times \mathrm{e}^{\mathrm{j} \mathbf{k}_{1 \leq \perp} \cdot \mathbf{R}_{j i \perp}} \mathrm{e}^{\mathrm{j} b K_{z} z_{j}} e_{b \eta} g\left(R_{j i}\right) \mathrm{d}^{3} \mathbf{R}_{j} \\
& +n_{0} \int_{D_{h}^{(-c)}} \mathrm{Q}\left(-k_{1} \mathbf{R}_{j i}\right) \mathrm{e}^{\mathrm{j} \mathbf{k}_{1 \mathrm{~s} \perp} \cdot \mathbf{R}_{j i \perp}} \overline{\mathrm{e}}_{(-c) \eta}\left(z_{j}\right) \mathrm{d}^{3} \mathbf{R}_{j} \\
& +n_{0} \int_{D_{h}^{c}-D_{2 a}\left(\mathbf{R}_{i}\right)} \mathrm{Q}\left(-k_{1} \mathbf{R}_{j i}\right) \mathrm{e}^{\mathrm{j} \mathbf{k}_{1 s \perp} \cdot \mathbf{R}_{j i \perp}} \overline{\mathrm{e}}_{c \eta}\left(z_{j}\right) g\left(R_{j i}\right) \mathrm{d}^{3} \mathbf{R}_{j} .
\end{aligned}
$$

For the hole-correction approximation, these integral equations can be discretized by employing the same approach as for the integral equation (195). The derived systems of equations are then 
solved together with the system of equations resulting from the discretization of Eqs. (229) and (230). The final system of equations for the two scalars (which provide the coefficients $x_{b \eta n}^{1,2}$ of $\mathrm{e}_{b \eta}$ ) and the discrete values $\overline{\mathrm{e}}_{c \eta}\left(z_{i}\right)$ is determined if the equations of the generalized Ewald-Oseen extinction theorem (229) and (230) can be reduced to two scalar equations; otherwise it is overdetermined. The answer to this question is an ongoing project of the authors. However, if the system of equations is overdetermined then it can be solved in the least squares sense with or without regularization. The regularization term may involve a diagonal matrix in order to control the magnitude of the two unknown scalars, and the first-order finite difference operator in order to control the smoothness of the discrete solutions $\overline{\mathrm{e}}_{c \eta}\left(z_{i}\right)$.

Finally, we note that as in Ref. [35], the solution representation for $\left\langle\mathrm{e}_{i \eta}\right\rangle_{i}\left(\mathbf{R}_{i}\right)$ in the domain $h \leq z_{i} \leq H-h$ can be assumed to be that of Eq. (203). In this case, we are led to (i) the generalized Lorenz-Lorentz law, as given by Eq. (206); (ii) the following equations of the generalized Ewald-Oseen extinction theorem:

$\mathrm{e}_{0 \eta}+n_{0} \mathrm{e}^{-\mathrm{j} k_{1 z} h} \sum_{p=1}^{N_{K}} \sum_{b= \pm} \mathrm{J}_{2 \mathrm{z} 0}^{b}\left(K_{p}\right) \mathrm{Te}_{p b \eta}+n_{0} \mathrm{j}_{\mathrm{z}}^{+}\left(\mathbf{R}_{i} ; \overline{\mathrm{e}}_{+\eta}\right)=0$

$\mathrm{e}^{\mathrm{j} k_{1 \mathrm{z}}(H-h)} \sum_{p=1}^{N_{K}} \sum_{b= \pm} \mathrm{e}^{\mathrm{j} b K_{p z} H} \mathrm{~J}_{2 \mathrm{z} H}^{b}\left(K_{p}\right) \mathrm{Te}_{p b \eta}+\mathrm{j}_{\mathrm{z}}^{-}\left(\mathbf{R}_{i} ; \overline{\mathrm{e}}_{-\eta}\right)=0 ;$

and (iii) an overdetermined system of equations for the $2 N_{K}$ scalars (which determine the coefficients $x_{p b \eta n}^{1,2}$ of $\mathrm{e}_{p b \eta}$ for $p=1, \ldots, N_{K}$ ) and the discrete values $\overline{\mathrm{e}}_{c \eta}\left(z_{i}\right)$.

\section{Acknowledgements}

We thank two anonymous reviewers for very insightful and stimulating comments. The authors are indebted to Gerhard Kristensson for his helpful comments and criticism during the course of this work. Adrian Doicu acknowledges the financial support from DLR programmatic (S5P KTR 2472 046) for the S5P algorithm development. Michael I. Mishchenko was supported by the NASA Radiation Science Program and the NASA Remote Sensing Theory Program.

\section{Appendix A}

The normalized associated Legendre functions are given by

$P_{n}^{|m|}(x)=\xi_{m n} \widetilde{P}_{n}^{|m|}(x)$,

where

$\widetilde{P}_{n}^{m}(x)=\left(1-x^{2}\right)^{m / 2} \frac{\mathrm{d}^{m}}{\mathrm{~d} x^{m}} \widetilde{P}_{n}(x)=\frac{\left(1-x^{2}\right)^{m / 2}}{2^{n} n !} \frac{\mathrm{d}^{m+n}}{\mathrm{~d} x^{m+n}}\left(x^{2}-1\right)^{n}$

are the conventional associated Legendre functions defined for positive and negative values of the azimuthal index $m$, and

$\xi_{m n}=\sqrt{\frac{2 n+1}{2} \cdot \frac{(n-|m|) !}{(n+|m|) !}}$

is a normalization constant. The angular functions $\pi_{n}^{|m|}(\theta)$ and $\tau_{n}^{|m|}(\theta)$ are given respectively, by

$\pi_{n}^{|m|}(\theta)=\frac{P_{n}^{|m|}(\cos \theta)}{\sin \theta}$

$\tau_{n}^{|m|}(\theta)=\frac{\mathrm{d}}{\mathrm{d} \theta} P_{n}^{|m|}(\cos \theta)$, and we have the orthogonality relations

$\int_{0}^{\pi} P_{n}^{|m|}(\cos \theta) P_{n^{\prime}}^{|m|}(\cos \theta) \sin \theta \mathrm{d} \theta=\delta_{n n^{\prime}}$,

$\int_{0}^{\pi}\left[\tau_{n}^{|m|}(\theta) \tau_{n^{\prime}}^{|m|}(\theta)+m^{2} \pi_{n}^{|m|}(\theta) \pi_{n^{\prime}}^{|m|}(\theta)\right]$

$\times \sin \theta \mathrm{d} \theta=n(n+1) \delta_{n n^{\prime}}$,

$\int_{0}^{\pi}\left[\pi_{n}^{|m|}(\theta) \tau_{n^{\prime}}^{|m|}(\theta)+\tau_{n}^{|m|}(\theta) \pi_{n^{\prime}}^{|m|}(\theta)\right] \sin \theta \mathrm{d} \theta=0$. by

The three normalized vector spherical harmonics are defined

$\mathbf{l}_{m n}(\theta, \varphi)=\frac{1}{\sqrt{2 \pi}} Y_{m n}(\theta, \varphi) \widehat{\mathbf{r}}$,

$\mathbf{n}_{m n}(\theta, \varphi)=\frac{1}{\sqrt{2 \pi n(n+1)}}\left[\tau_{n}^{|m|}(\theta) \widehat{\boldsymbol{\theta}}(\widehat{\mathbf{r}})+\mathrm{j} m \pi_{n}^{|m|}(\theta) \widehat{\boldsymbol{\varphi}}(\widehat{\mathbf{r}})\right] \mathrm{e}^{\mathrm{j} m \varphi}$,

$\mathbf{m}_{m n}(\theta, \varphi)=\frac{1}{\sqrt{2 \pi n(n+1)}}\left[\mathrm{j} m \pi_{n}^{|m|}(\theta) \widehat{\boldsymbol{\theta}}(\widehat{\mathbf{r}})-\tau_{n}^{|m|}(\theta) \widehat{\boldsymbol{\varphi}}(\widehat{\mathbf{r}})\right] \mathrm{e}^{\mathrm{j} m \varphi}$,

where $(\theta, \varphi)$ are the spherical angles of the direction $\widehat{\mathbf{r}}$, and

$Y_{m n}(\theta, \varphi)=P_{n}^{|m|}(\cos \theta) \mathrm{e}^{\mathrm{j} m \varphi}$

are the (normalized) spherical harmonics. They satisfy the orthogonality relation

$\int_{0}^{2 \pi} \int_{0}^{\pi} \mathbf{v}_{m n}^{\alpha}(\theta, \varphi) \cdot \mathbf{v}_{-m^{\prime} n^{\prime}}^{\beta}(\theta, \varphi) \sin \theta \mathrm{d} \theta \mathrm{d} \varphi=\delta_{\alpha \beta} \delta_{m m^{\prime}} \delta_{n n^{\prime}}$,

where $\alpha, \beta=1,2,3, \mathbf{v}_{m n}^{1}(\theta, \varphi)=\mathbf{l}_{m n}(\theta, \varphi), \mathbf{v}_{m n}^{2}(\theta, \varphi)=\mathbf{n}_{m n}(\theta, \varphi)$, and $\mathbf{v}_{m n}^{3}(\theta, \varphi)=\mathbf{m}_{m n}(\theta, \varphi)$.

The normalized vector spherical wave functions are defined by

$\mathbf{L}_{m n}^{1,3}(k \mathbf{r})=\left[z_{n}^{1,3}(k r)\right]^{\prime} \mathbf{l}_{m n}(\theta, \varphi)+\sqrt{n(n+1)} \frac{z_{n}^{1,3}(k r)}{k r} \mathbf{n}_{m n}(\theta, \varphi)$,

$\mathbf{M}_{m n}^{1,3}(k \mathbf{r})=z_{n}^{1,3}(k r) \mathbf{m}_{m n}(\theta, \varphi)$,

$\mathbf{N}_{m n}^{1,3}(k \mathbf{r})=\sqrt{n(n+1)} \frac{z_{n}^{1,3}(k r)}{k r} \mathbf{l}_{m n}(\theta, \varphi)+\frac{\left[k r z_{n}^{1,3}(k r)\right]^{\prime}}{k r} \mathbf{n}_{m n}(\theta, \varphi)$,

where $u_{m n}^{1,3}(k \mathbf{r})=z_{n}^{1,3}(k r) Y_{m n}(\theta, \varphi)$ are the spherical wave functions, $z_{n}^{1}(k r)$ stands for the spherical Bessel functions $j_{n}(k r)$, $z_{n}^{3}(k r)$ stands for the spherical Hankel functions of the first kind $h_{n}^{(1)}(k r)$,

$\left[z_{n}^{1,3}(k r)\right]^{\prime}=\frac{\mathrm{d}}{\mathrm{d}(k r)}\left[z_{n}^{1,3}(k r)\right]$

and

$\left[k r z_{n}^{1,3}(k r)\right]^{\prime}=\frac{\mathrm{d}}{\mathrm{d}(k r)}\left[k r z_{n}^{1,3}(k r)\right]=\frac{\mathrm{d}}{\mathrm{d} r}\left[r z_{n}^{1,3}(k r)\right]$. 
The symmetry relation

$P_{n}^{|m|}(-\cos \theta)=(-1)^{n-|m|} P_{n}^{|m|}(\cos \theta)$,

yields

$\pi_{n}^{|m|}(\pi-\theta)=(-1)^{n-|m|} \pi_{n}^{|m|}(\theta)$,

$\tau_{n}^{|m|}(\pi-\theta)=(-1)^{n-|m|+1} \tau_{n}^{|m|}(\theta)$.

By virtue of Eqs. (248), (249), and (250), and the relations $\widehat{\boldsymbol{\theta}}(-\widehat{\mathbf{r}})=$ $\widehat{\boldsymbol{\theta}}(\widehat{\mathbf{r}})$ and $\widehat{\varphi}(-\widehat{\mathbf{r}})=-\widehat{\varphi}(\widehat{\mathbf{r}})$, we get

$\mathbf{l}_{m n}(-\widehat{\mathbf{r}})=(-1)^{n+1} \mathbf{l}_{m n}(\widehat{\mathbf{r}})$,

$\mathbf{m}_{m n}(-\widehat{\mathbf{r}})=(-1)^{n} \mathbf{m}_{m n}(\widehat{\mathbf{r}})$

$\mathbf{n}_{m n}(-\widehat{\mathbf{r}})=(-1)^{n+1} \mathbf{n}_{m n}(\widehat{\mathbf{r}})$,

and moreover,

$\mathbf{M}_{m n}^{3}\left(-k_{1} \mathbf{r}\right)=(-1)^{n} \mathbf{M}_{m n}^{3}\left(k_{1} \mathbf{r}\right)$,

$\mathbf{N}_{m n}^{3}\left(-k_{1} \mathbf{r}\right)=(-1)^{n+1} \mathbf{N}_{m n}^{3}\left(k_{1} \mathbf{r}\right)$.

\section{Appendix B}

The addition theorem for vector spherical harmonics can be obtained from the addition theorem for scalar spherical harmonics

$P_{n}(\cos \Theta)=\sum_{m=-n}^{n} \sqrt{\frac{2}{2 n+1}} P_{n}^{|m|}(\cos \theta) P_{n}^{|m|}\left(\cos \theta^{\prime}\right) \mathrm{e}^{\mathrm{j} m\left(\varphi-\varphi^{\prime}\right)}$,

where

$\cos \Theta=\cos \theta \cos \theta^{\prime}+\sin \theta \sin \theta^{\prime} \cos \left(\varphi-\varphi^{\prime}\right)$.

Using the identities

$\frac{\partial^{2} P_{n}}{\partial \theta \partial \theta^{\prime}}=P_{n}^{\prime \prime}(x)\left[\widehat{\boldsymbol{\theta}}(\widehat{\mathbf{k}}) \cdot \widehat{\mathbf{k}}^{\prime}\right]\left[\widehat{\mathbf{k}} \cdot \widehat{\boldsymbol{\theta}}\left(\widehat{\mathbf{k}}^{\prime}\right)\right]+P_{n}^{\prime}(x)\left[\widehat{\boldsymbol{\theta}}(\widehat{\mathbf{k}}) \cdot \widehat{\boldsymbol{\theta}}\left(\widehat{\mathbf{k}}^{\prime}\right)\right]$,

$\frac{1}{\sin \theta^{\prime}} \frac{\partial^{2} P_{n}}{\partial \theta \partial \varphi^{\prime}}=P_{n}^{\prime \prime}(x)\left[\widehat{\boldsymbol{\theta}}(\widehat{\mathbf{k}}) \cdot \widehat{\mathbf{k}}^{\prime}\right]\left[\widehat{\mathbf{k}} \cdot \widehat{\boldsymbol{\varphi}}\left(\widehat{\mathbf{k}}^{\prime}\right)\right]+P_{n}^{\prime}(x)\left[\widehat{\boldsymbol{\theta}}(\widehat{\mathbf{k}}) \cdot \widehat{\boldsymbol{\varphi}}\left(\widehat{\mathbf{k}}^{\prime}\right)\right]$,

$\frac{1}{\sin \theta} \frac{\partial^{2} P_{n}}{\partial \varphi \partial \theta^{\prime}}=P_{n}^{\prime \prime}(x)\left[\widehat{\boldsymbol{\varphi}}(\widehat{\mathbf{k}}) \cdot \widehat{\mathbf{k}}^{\prime}\right]\left[\widehat{\mathbf{k}} \cdot \widehat{\boldsymbol{\theta}}\left(\widehat{\mathbf{k}}^{\prime}\right)\right]+P_{n}^{\prime}(x)\left[\widehat{\boldsymbol{\varphi}}(\widehat{\mathbf{k}}) \cdot \widehat{\boldsymbol{\theta}}\left(\widehat{\mathbf{k}}^{\prime}\right)\right]$,

$\frac{1}{\sin \theta \sin \theta^{\prime}} \frac{\partial^{2} P_{n}}{\partial \varphi \partial \varphi^{\prime}}=P_{n}^{\prime \prime}(x)\left[\widehat{\varphi}(\widehat{\mathbf{k}}) \cdot \widehat{\mathbf{k}}^{\prime}\right]\left[\widehat{\mathbf{k}} \cdot \widehat{\boldsymbol{\varphi}}\left(\widehat{\mathbf{k}}^{\prime}\right)\right]$

$$
+P_{n}^{\prime}(x)\left[\widehat{\boldsymbol{\varphi}}(\widehat{\mathbf{k}}) \cdot \widehat{\boldsymbol{\varphi}}\left(\widehat{\mathbf{k}}^{\prime}\right)\right]
$$

with $\widehat{\mathbf{k}}=\widehat{\mathbf{k}}(\theta, \varphi), \widehat{\mathbf{k}}^{\prime}=\widehat{\mathbf{k}}^{\prime}\left(\theta^{\prime}, \varphi^{\prime}\right)$, and $x=\widehat{\mathbf{k}} \cdot \widehat{\mathbf{k}}^{\prime}=\cos \Theta$, we obtain $[5,23]$

$\sum_{m} \mathbf{l}_{m n}(\widehat{\mathbf{k}}) \otimes \mathbf{l}_{-m n}\left(\widehat{\mathbf{k}}^{\prime}\right)=\frac{1}{2 \pi} \sqrt{\frac{2 n+1}{2}} P_{n}(x) \widehat{\mathbf{k}} \otimes \widehat{\mathbf{k}}^{\prime}$,

$\sum_{m} \mathbf{l}_{m n}(\widehat{\mathbf{k}}) \otimes \mathbf{m}_{-m n}\left(\widehat{\mathbf{k}}^{\prime}\right)=\chi_{n}\left[\frac{1}{\sin \theta^{\prime}} \frac{\partial P_{n}}{\partial \varphi^{\prime}} \widehat{\mathbf{k}} \otimes \widehat{\boldsymbol{\theta}}\left(\widehat{\mathbf{k}}^{\prime}\right)-\frac{\partial P_{n}}{\partial \theta^{\prime}} \widehat{\mathbf{k}} \otimes \widehat{\boldsymbol{\varphi}}\left(\widehat{\mathbf{k}}^{\prime}\right)\right]$,

$\sum_{m} \mathbf{l}_{m n}(\widehat{\mathbf{k}}) \otimes \mathbf{n}_{-m n}\left(\widehat{\mathbf{k}}^{\prime}\right)=\chi_{n}\left[\frac{\partial P_{n}}{\partial \theta^{\prime}} \widehat{\mathbf{k}} \otimes \widehat{\boldsymbol{\theta}}\left(\widehat{\mathbf{k}}^{\prime}\right)+\frac{1}{\sin \theta^{\prime}} \frac{\partial P_{n}}{\partial \varphi^{\prime}} \widehat{\mathbf{k}} \otimes \widehat{\boldsymbol{\varphi}}\left(\widehat{\mathbf{k}}^{\prime}\right)\right]$,

$$
\sum_{m} \mathbf{m}_{m n}(\widehat{\mathbf{k}}) \otimes \mathbf{I}_{-m n}\left(\widehat{\mathbf{k}}^{\prime}\right)=\chi_{n}\left[\frac{1}{\sin \theta} \frac{\partial P_{n}}{\partial \varphi} \widehat{\boldsymbol{\theta}}(\widehat{\mathbf{k}}) \otimes \widehat{\mathbf{k}}^{\prime}-\frac{\partial P_{n}}{\partial \theta} \widehat{\boldsymbol{\varphi}}(\widehat{\mathbf{k}}) \otimes \widehat{\mathbf{k}}^{\prime}\right],
$$

$$
\sum_{m} \mathbf{n}_{m n}(\widehat{\mathbf{k}}) \otimes \mathbf{l}_{-m n}\left(\widehat{\mathbf{k}}^{\prime}\right)=\chi_{n}\left[\frac{\partial P_{n}}{\partial \theta} \widehat{\boldsymbol{\theta}}(\widehat{\mathbf{k}}) \otimes \widehat{\mathbf{k}}^{\prime}+\frac{1}{\sin \theta} \frac{\partial P_{n}}{\partial \varphi} \widehat{\boldsymbol{\varphi}}(\widehat{\mathbf{k}}) \otimes \widehat{\mathbf{k}}^{\prime}\right],
$$

and

$$
\begin{aligned}
\sum_{m} \mathbf{m}_{m n}(\widehat{\mathbf{k}}) \otimes \mathbf{m}_{-m n}\left(\widehat{\mathbf{k}}^{\prime}\right) & =\chi_{n}\left[\frac{1}{\sin \theta \sin \theta^{\prime}} \frac{\partial^{2} P_{n}}{\partial \varphi \partial \varphi^{\prime}} \widehat{\boldsymbol{\theta}}(\widehat{\mathbf{k}}) \otimes \widehat{\boldsymbol{\theta}}\left(\widehat{\mathbf{k}}^{\prime}\right)\right. \\
& -\frac{1}{\sin \theta} \frac{\partial^{2} P_{n}}{\partial \varphi \partial \theta^{\prime}} \widehat{\boldsymbol{\theta}}(\widehat{\mathbf{k}}) \otimes \widehat{\boldsymbol{\varphi}}\left(\widehat{\mathbf{k}}^{\prime}\right) \\
& -\frac{1}{\sin \theta^{\prime}} \frac{\partial^{2} P_{n}}{\partial \theta \partial \varphi^{\prime}} \widehat{\boldsymbol{\varphi}}(\widehat{\mathbf{k}}) \otimes \widehat{\boldsymbol{\theta}}\left(\widehat{\mathbf{k}}^{\prime}\right) \\
& \left.+\frac{\partial^{2} P_{n}}{\partial \theta \partial \theta^{\prime}} \widehat{\boldsymbol{\varphi}}(\widehat{\mathbf{k}}) \otimes \widehat{\boldsymbol{\varphi}}\left(\widehat{\mathbf{k}}^{\prime}\right)\right]
\end{aligned}
$$

$$
\begin{aligned}
\sum_{m} \mathbf{n}_{m n}(\widehat{\mathbf{k}}) \otimes \mathbf{n}_{-m n}\left(\widehat{\mathbf{k}}^{\prime}\right) & =\chi_{n}\left[\frac{\partial^{2} P_{n}}{\partial \theta \partial \theta^{\prime}} \widehat{\boldsymbol{\theta}}(\widehat{\mathbf{k}}) \otimes \widehat{\boldsymbol{\theta}}\left(\widehat{\mathbf{k}}^{\prime}\right)\right. \\
& +\frac{1}{\sin \theta^{\prime}} \frac{\partial^{2} P_{n}}{\partial \theta \partial \varphi^{\prime}} \widehat{\boldsymbol{\theta}}(\widehat{\mathbf{k}}) \otimes \widehat{\boldsymbol{\varphi}}\left(\widehat{\mathbf{k}}^{\prime}\right) \\
& +\frac{1}{\sin \theta} \frac{\partial^{2} P_{n}}{\partial \varphi \partial \theta^{\prime}} \widehat{\boldsymbol{\varphi}}(\widehat{\mathbf{k}}) \otimes \widehat{\boldsymbol{\theta}}\left(\widehat{\mathbf{k}}^{\prime}\right) \\
& \left.+\frac{1}{\sin \theta \sin \theta^{\prime}} \frac{\partial^{2} P_{n}}{\partial \varphi \partial \varphi^{\prime}} \widehat{\boldsymbol{\varphi}}(\widehat{\mathbf{k}}) \otimes \widehat{\boldsymbol{\varphi}}\left(\widehat{\mathbf{k}}^{\prime}\right)\right],
\end{aligned}
$$

$$
\begin{aligned}
\sum_{m} \mathbf{m}_{m n}(\widehat{\mathbf{k}}) \otimes \mathbf{n}_{-m n}\left(\widehat{\mathbf{k}}^{\prime}\right) & =\chi_{n}\left[\frac{1}{\sin \theta} \frac{\partial^{2} P_{n}}{\partial \varphi \partial \theta^{\prime}} \widehat{\boldsymbol{\theta}}(\widehat{\mathbf{k}}) \otimes \widehat{\boldsymbol{\theta}}\left(\widehat{\mathbf{k}}^{\prime}\right)\right. \\
& +\frac{1}{\sin \theta \sin \theta^{\prime}} \frac{\partial^{2} P_{n}}{\partial \varphi \partial \varphi^{\prime}} \widehat{\boldsymbol{\theta}}(\widehat{\mathbf{k}}) \otimes \widehat{\boldsymbol{\varphi}}\left(\widehat{\mathbf{k}}^{\prime}\right) \\
& -\frac{\partial^{2} P_{n}}{\partial \theta \partial \theta^{\prime}} \widehat{\boldsymbol{\varphi}}(\widehat{\mathbf{k}}) \otimes \widehat{\boldsymbol{\theta}}\left(\widehat{\mathbf{k}}^{\prime}\right) \\
& \left.-\frac{1}{\sin \theta^{\prime}} \frac{\partial^{2} P_{n}}{\partial \theta \partial \varphi^{\prime}} \widehat{\boldsymbol{\varphi}}(\widehat{\mathbf{k}}) \otimes \widehat{\boldsymbol{\varphi}}\left(\widehat{\mathbf{k}}^{\prime}\right)\right],
\end{aligned}
$$

$$
\begin{aligned}
\sum_{m} \mathbf{n}_{m n}(\widehat{\mathbf{k}}) \otimes \mathbf{m}_{-m n}\left(\widehat{\mathbf{k}}^{\prime}\right) & =\chi_{n}\left[\frac{1}{\sin \theta^{\prime}} \frac{\partial^{2} P_{n}}{\partial \theta \partial \varphi^{\prime}} \widehat{\boldsymbol{\theta}}(\widehat{\mathbf{k}}) \otimes \widehat{\boldsymbol{\theta}}\left(\widehat{\mathbf{k}}^{\prime}\right)\right. \\
& -\frac{\partial^{2} P_{n}}{\partial \theta \partial \theta^{\prime}} \widehat{\boldsymbol{\theta}}(\widehat{\mathbf{k}}) \otimes \widehat{\boldsymbol{\varphi}}\left(\widehat{\mathbf{k}}^{\prime}\right) \\
& +\frac{1}{\sin \theta \sin \theta^{\prime}} \frac{\partial^{2} P_{n}}{\partial \varphi \partial \varphi^{\prime}} \widehat{\boldsymbol{\varphi}}(\widehat{\mathbf{k}}) \otimes \widehat{\boldsymbol{\theta}}\left(\widehat{\mathbf{k}}^{\prime}\right) \\
& \left.-\frac{1}{\sin \theta} \frac{\partial^{2} P_{n}}{\partial \varphi \partial \theta^{\prime}} \widehat{\boldsymbol{\varphi}}(\widehat{\mathbf{k}}) \otimes \widehat{\boldsymbol{\varphi}}\left(\widehat{\mathbf{k}}^{\prime}\right)\right],
\end{aligned}
$$

where

$\chi_{n}=\frac{1}{2 \pi n(n+1)} \sqrt{\frac{2 n+1}{2}}$.

The addition theorem for vector spherical harmonics in the case $\varphi=\varphi^{\prime}$ becomes

$$
\begin{aligned}
\sum_{m} \mathbf{m}_{m n}(\widehat{\mathbf{k}}) \otimes \mathbf{m}_{-m n}\left(\widehat{\mathbf{k}}^{\prime}\right) & =\chi_{n}\left[\frac{1}{\sin \theta \sin \theta^{\prime}} \frac{\partial^{2} P_{n}(x)}{\partial \varphi^{2}} \widehat{\boldsymbol{\theta}}(\widehat{\mathbf{k}}) \otimes \widehat{\boldsymbol{\theta}}\left(\widehat{\mathbf{k}}^{\prime}\right)\right. \\
& \left.+\frac{\partial^{2} P_{n}(x)}{\partial \theta \partial \theta^{\prime}} \widehat{\boldsymbol{\varphi}}(\widehat{\mathbf{k}}) \otimes \widehat{\boldsymbol{\varphi}}\left(\widehat{\mathbf{k}}^{\prime}\right)\right]
\end{aligned}
$$




$$
\begin{aligned}
\sum_{m} \mathbf{n}_{m n}(\widehat{\mathbf{k}}) \otimes \mathbf{n}_{-m n}\left(\widehat{\mathbf{k}}^{\prime}\right) & =\chi_{n}\left[\frac{\partial^{2} P_{n}(x)}{\partial \theta \partial \theta^{\prime}} \widehat{\boldsymbol{\theta}}(\widehat{\mathbf{k}}) \otimes \widehat{\boldsymbol{\theta}}\left(\widehat{\mathbf{k}}^{\prime}\right)\right. \\
& \left.+\frac{1}{\sin \theta \sin \theta^{\prime}} \frac{\partial^{2} P_{n}(x)}{\partial \varphi^{2}} \widehat{\boldsymbol{\varphi}}(\widehat{\mathbf{k}}) \otimes \widehat{\boldsymbol{\varphi}}\left(\widehat{\mathbf{k}}^{\prime}\right)\right]
\end{aligned}
$$

$$
\begin{aligned}
\sum_{m} \mathbf{m}_{m n}(\widehat{\mathbf{k}}) \otimes \mathbf{n}_{-m n}\left(\widehat{\mathbf{k}}^{\prime}\right) & =\chi_{n}\left[\frac{1}{\sin \theta \sin \theta^{\prime}} \frac{\partial^{2} P_{n}(x)}{\partial \varphi^{2}} \widehat{\boldsymbol{\theta}}(\widehat{\mathbf{k}}) \otimes \widehat{\boldsymbol{\varphi}}\left(\widehat{\mathbf{k}}^{\prime}\right)\right. \\
& \left.-\frac{\partial^{2} P_{n}(x)}{\partial \theta \partial \theta^{\prime}} \widehat{\boldsymbol{\varphi}}(\widehat{\mathbf{k}}) \otimes \widehat{\boldsymbol{\theta}}\left(\widehat{\mathbf{k}}^{\prime}\right)\right]
\end{aligned}
$$

$$
\begin{aligned}
\sum_{m} \mathbf{n}_{m n}(\widehat{\mathbf{k}}) \otimes \mathbf{m}_{-m n}\left(\widehat{\mathbf{k}}^{\prime}\right) & =\chi_{n}\left[-\frac{\partial^{2} P_{n}(x)}{\partial \theta \partial \theta^{\prime}} \widehat{\boldsymbol{\theta}}(\widehat{\mathbf{k}}) \otimes \widehat{\boldsymbol{\varphi}}\left(\widehat{\mathbf{k}}^{\prime}\right)\right. \\
& \left.+\frac{1}{\sin \theta \sin \theta^{\prime}} \frac{\partial^{2} P_{n}(x)}{\partial \varphi^{2}} \widehat{\boldsymbol{\varphi}}(\widehat{\mathbf{k}}) \otimes \widehat{\boldsymbol{\theta}}\left(\widehat{\mathbf{k}}^{\prime}\right)\right]
\end{aligned}
$$

where $P_{n}(x)=P_{n}^{0}(x)$ are the Legendre polynomials and $x=\widehat{\mathbf{k}} \cdot \widehat{\mathbf{k}}^{\prime}=$ $\cos \left(\theta-\theta^{\prime}\right)$. By means of the recurrence relations for the associated Legendre functions, the partial derivatives in Eqs. (271)-(274) can be readily computed; they are given by

$\frac{1}{\sin \theta \sin \theta^{\prime}} \frac{\partial^{2} P_{n}(x)}{\partial \varphi^{2}}=\sqrt{n(n+1)} M_{n}\left(\widehat{\mathbf{k}} \cdot \widehat{\mathbf{k}}^{\prime}\right)$,

$\frac{\partial^{2} P_{n}(x)}{\partial \theta \partial \theta^{\prime}}=-\sqrt{n(n+1)} N_{n}\left(\widehat{\mathbf{k}} \cdot \widehat{\mathbf{k}}^{\prime}\right)$,

where

$M_{n}\left(\widehat{\mathbf{k}} \cdot \widehat{\mathbf{k}}^{\prime}\right)=\pi_{n}^{1}(x)$

$N_{n}\left(\widehat{\mathbf{k}} \cdot \widehat{\mathbf{k}}^{\prime}\right)=x \pi_{n}^{1}(x)-\sqrt{n(n+1)} P_{n}(x)$

and $\pi_{n}^{1}(x)=P_{n}^{1}(x) / \sqrt{1-x^{2}}$.

\section{Appendix C}

Consider the integral

$I_{m n}(z)=k^{2} \int_{\sqrt{a^{2}-z^{2}}}^{\infty} h_{n}\left(k \sqrt{\rho^{2}+z^{2}}\right) P_{n}^{|m|}\left(\frac{z}{\sqrt{\rho^{2}+z^{2}}}\right) J_{m}(k \eta \rho) \rho \mathrm{d} \rho$,

where $\eta=\sin \theta_{0}$. As compared to Eq. (202), note the mild change in notation $2 a \rightarrow a$.

Using the result [38]

$$
-\frac{1}{k} \frac{\partial}{\partial z} u_{m n}^{3}=\sqrt{\frac{(n+1)^{2}-m^{2}}{(2 n+1)(2 n+3)}} u_{m, n+1}-\sqrt{\frac{n^{2}-m^{2}}{4 n^{2}-1}} u_{m, n-1},
$$

the following recurrence relation

$$
\begin{aligned}
I_{m, n+1}(z) & =\sqrt{\frac{(2 n+1)(2 n+3)}{(n+1)^{2}-m^{2}}}(k z) h_{n}(k a) P_{n}^{|m|}\left(\frac{z}{a}\right) J_{m}\left(k \eta \sqrt{a^{2}-z^{2}}\right) \\
& -\sqrt{\frac{(2 n+1)(2 n+3)}{(n+1)^{2}-m^{2}}} \frac{1}{k} I_{m n}^{\prime}(z) \\
& +\sqrt{\frac{(2 n+3)\left(n^{2}-m^{2}\right)}{(2 n-1)\left[(n+1)^{2}-m^{2}\right]}} I_{m, n-1}(z)
\end{aligned}
$$

readily follows. For normal incidence, we have $\eta=0$, and from $J_{m}(0)=1$ for $m=0$ and $J_{m}(0)=0$ for $m \geq 1$, we get $m=0$. In this case, Eq. (281) becomes

$$
\begin{aligned}
I_{0, n+1}(z) & =\frac{\sqrt{(2 n+1)(2 n+3)}}{n+1}(k z) h_{n}(k a) P_{n}\left(\frac{z}{a}\right) \\
& -\frac{\sqrt{(2 n+1)(2 n+3)}}{n+1} \frac{1}{k} I_{0 n}^{\prime}(z) \\
& +\frac{n}{n+1} \sqrt{\frac{2 n+3}{2 n-1}} I_{0, n-1}(z),
\end{aligned}
$$

and as shown by Kristensson [33,34], the recurrence (282) has a surprisingly and elegant solution given by

$$
\begin{aligned}
I_{0 n}(z) & =-(k a) h_{n+1}(k a) P_{n}\left(\frac{k}{a}\right) \\
& +\sum_{l=0}^{[n / 2]}(-1)^{l} \sqrt{(2 n+1)(2 n-4 l+1)} h_{n-2 l}(k a) P_{n-2 l}\left(\frac{z}{a}\right) .
\end{aligned}
$$

The derivation given in Ref. [34] uses the fact that the term proportional to $(k z) P_{n}(z / a)$ on the right-hand side of Eq. (282) implies that $I_{0 n}(z)$ is a polynomial in $z$ of the order $n$, so that $I_{0 n}(z)$ can be expressed as a finite series of Legendre polynomials, i.e.,

$I_{0 n}(z)=\sum_{l=0}^{[n / 2]} a_{n l} P_{n-2 l}\left(\frac{z}{a}\right)$.

Unfortunately, for oblique incidence, the term which is proportional to

$$
(k z) P_{n}^{|m|}(z / a) J_{m}\left(k \eta \sqrt{a^{2}-z^{2}}\right)
$$

on the right-hand side of Eq. (281) does not lead to this conclusion. For this reason, the method presented in Ref. [34] cannot be applied directly, and more effort should be made to design an analytical method for computing the integral $I_{m n}(z)$.

However, the integral $I_{m n}(z)$ can be numerically estimated by computing the approximations

$I_{m n}^{1}(z)=\int_{\sqrt{a^{2}-z^{2}}}^{H} f_{m n}(z, \rho) \mathrm{d} \rho$,

$I_{m n}^{J}(z)=\int_{\sqrt{a^{2}-z^{2}}}^{H} f_{m n}(z, \rho) \mathrm{d} \rho+\sum_{j=2}^{J} \int_{(j-1) H}^{j H} f_{m n}(z, \rho) \mathrm{d} \rho, J \geq 2$

by means of Romberg integration. Here, $f_{m n}(z, \rho)$ stands for the integrand in Eq. (279), and $H$ is the length of each integration interval. In order to increase the numerical efficiency, the asymptotic representations for the spherical Hankel and cylindrical Bessel functions

$h_{n}(x)=\frac{1}{x} \mathrm{e}^{\mathrm{j}\left(x-\frac{n+1}{2} \pi\right)}$ with $x=k \sqrt{\rho^{2}+z^{2}}$, and

$J_{m}(x)=\sqrt{\frac{2}{\pi x}} \cos \left[x-\frac{(2 m+1) \pi}{4}\right]$ with $x=k \eta \rho$,

can be used for $\rho \geq \rho_{\max }$ with a sufficiently large $\rho_{\max }$, while in order to speed up the convergence, a small imaginary part can be added to the wavenumber $k$, i.e., $k \rightarrow k+\mathrm{j} \varepsilon$. As an example, we illustrate in Fig. 10 the approximations $I_{m n}^{J}(z)$ in the case $m=0$ and $n=4$ for normal and oblique incidence. From these plots we see that although $J$ should be large in order to achieve a reasonable accuracy, the integral $I_{m n}(z)$ can, in principle, be computed by the above numerical algorithm. 


\section{References}

[1] Ishimaru A. Wave propagation and scattering in random media. Vol. 1. Single scattering and transport theory. New York: Academic Press; 1978.

[2] Ishimaru A. Wave propagation and scattering in random media. Vol. 2. Multiple scattering, turbulence, rough surfaces, and remote sensing. New York: Academic Press; 1978.

[3] Tsang L, Kong JA, Ding KH. Scattering of electromagnetic waves: theories and applications. New York: Wiley; 2000.

[4] Tsang L, Kong JA, Ding KH. Scattering of electromagnetic waves: numerical simulations. New York: Wiley; 2001.

[5] Tsang L, Kong JA. Scattering of electromagnetic waves: advanced topics. New York: Wiley; 2001.

[6] Martin PA. Multiple scattering: interaction of time-harmonic waves with $\mathrm{N}$ obstacles. Cambridge, UK: Cambridge University Press; 2006.

[7] Mishchenko MI, Travis LD, Lacis AA. Multiple scattering of light by particles: radiative transfer and coherent backscattering. Cambridge, UK: Cambridge University Press; 2006.

[8] Mishchenko MI. Electromagnetic scattering by particles and particle groups: an introduction. Cambridge, UK: Cambridge University Press; 2014. https://www. giss.nasa.gov/staff/mmishchenko/publications/Book_4.pdf

[9] Foldy LL. The multiple scattering of waves. I. General theory of isotropic scattering by randomly distributed scatterers. Phys Rev 1945;67:107-19.

[10] Lax M. Multiple scattering of waves. Rev Mod Phys 1951;23:287-310.

[11] Lax M. Multiple scattering of waves. II. Effective field in dense systems. Phys Rev 1952;85:621-9.

[12] Twersky V. On scattering and reflection of sound by rough surfaces. J Acoust Soc Am 1957;29:209-25.

[13] Waterman PC, Truell R. Multiple scattering of waves. J Math Phys $1961: 2: 512-37$

[14] Fikioris JG, Waterman PC. Multiple scattering of waves. II. "Hole corrections" in the scalar case. J Math Phys 1964;5:1413-20.

[15] Twersky V. On propagation in random media of discrete scatterers. Proc Symp Appl Math 1964;16:84-116.

[16] Twersky V. Coherent scalar field in pair-correlated random distributions of aligned scatterers. J Math Phys 1964;5:1619-28.

[17] Fikioris JG, Waterman PC. Multiple scattering of waves. III. The electromagnetic case. J Quant Spectrosc Radiat Transfer 2013;123:8-16.

[18] Varadan VK, Bringi VN, Varadan VV. Coherent electromagnetic wave propagation through randomly distributed dielectric scatterers. Phys Rev D 1979;19:2480-9.

[19] Varadan VK, Bringi VN, Varadan VV, Ishimaru A. Multiple scattering theory for waves in discrete random media and comparison with experiments. Radio Sci 1983;18:321-7.

[20] Varadan VV, Varadan VK. Multiple scattering of electromagnetic waves by randomly distributed and oriented dielectric scatters. Phys Rev D 1980;21:388-94.

[21] Bringi VN, Seliga TA, Varadan VK, Varadan VV. Bulk propagation characteristics of discrete random media. In: Chow PL, Kohler WE, Papanicolaou GC, editors. Multiple scattering and waves in random media. Amsterdam: North-Holland; 1981. p. 43-75.
[22] Bringi VN, Varadan VV, Varadan VK. Coherent wave attenuation by a random distribution of particles. Radio Sci 1982;17:946-52.

[23] Tsang L, Kong JA. Scattering of electromagnetic waves from a half space of densely distributed dielectric scatterers. Radio Sci 1983;18:1260-72.

[24] Mishchenko MI. Vector radiative transfer equation for arbitrarily shaped and arbitrarily oriented particles: a microphysical derivation from statistical electromagnetics. Appl Opt 2002;41:7114-34.

[25] Mishchenko MI, Dlugach JM, Yurkin MA, Bi L, Cairns B, Liu L, Panetta RL, Travis LD, Yang P, Zakharova NT. First-principles modeling of electromagnetic scattering by discrete and discretely heterogeneous random media. Phys Rep 2016;632:1-75

[26] Mishchenko MI, Yurkin MA. Impressed sources and fields in the volume-integral-equation formulation of electromagnetic scattering by a finite object: a tutorial. J Quant Spectrosc Radiat Transfer 2018;214:158-67.

[27] Tsang L, Kong JA. Multiple scattering of electromagnetic waves by random distributions of discrete scatterers with coherent potential and quantum mechanical formalism. J Appl Phys 1980;51:3465-85.

[28] Ornstein LS, Zernike F. Accidental deviations of density and opalescence at the critical point of a single substance. Proc Neth Acad Arts Sci 1914;17:793-806.

[29] Percus JK, Yevick GJ. Analysis of classical statistical mechanics by means of collective coordinates. Phys Rev 1958:110:1-13.

[30] Stratton JA. Electromagnetic theory. New York: McGraw-Hill; 1941.

[31] Boström A, Kristensson G, Ström S. Transformation properties of plane, spherical and cylindrical scalar and vector wave functions. In: Varadan VV, Lakhtakia A, Varadan VK, editors. Field representations and introduction to scattering. Amsterdam: Elsevier; 1991. p. 165-210.

[32] Gustavsson M, Kristensson G, Wellander N. Multiple scattering by a collection of randomly located obstacles - numerical implementation of the coherent fields. J Quant Spectrosc Radiat Transfer 2016;185:95-100.

[33] Kristensson G. Coherent scattering by a collection of randomly located obstacles - an alternative integral equation formulation. J Quant Spectrosc Radiat Transfer 2015;164:97-108.

[34] Kristensson G. Evaluation of an integral relevant to multiple scattering by randomly distributed obstacles. Technical report LUTEDX/(TEAT-7228)/110/(2014), Lund University, Department of Electrical and Information Technology, P.O. Box 118, S-221 00 Lund, Sweden; 2014. http://www.eit.lth.se

[35] Gower AL, Parnell WJ, Abrahams ID. Multiple waves propagate in random particulate materials. 2018. arXiv:1810.10816.

[36] Linton CM, Martin PA. Multiple scattering by random configurations of circular cylinders: second-order corrections for the effective wavenumber. J Acoust Soc Am 2005;117:3413-23.

[37] Martin PA. Multiple scattering by random configurations of circular cylinders: reflection, transmission, and effective interface conditions. J Acoust Soc Am 2011;129:1685-95.

[38] Martin PA. Multiple scattering: interaction of time-harmonic waves with $\mathrm{N}$ obstacles. Cambridge, UK: Cambridge University Press; 2006. 